Matlanal Bureau of Standards

L Library, N.W. Bldg

; 191962

\title{
NBS
}

Eechnical Note

\section{INFORMATION SELECTION SYSTEMS RETRIEVING REPLICA COPIES:}

\section{A STATE-OF-THE-ART REPORT}

THOMAS C. BAGG AND MARY ELIZABETH STEVENS

U. S. DEPARTMENT OF COMMERCE NATIONAL BUREAU OF STANDARDS 


\section{THE NATIONAL BUREAU OF STANDARDS}

\section{Functions and Activitios}

The functions of the National Bureau of Standards are set forth in the Act of Congress, March 3, 1901, as amended by Congress in Public Law 619, 1950. These include the development and maintenance of the national standards of measurement and the provision of means and methods for making measurements consistent with these standards; the determination of physical constants and properties of materials; the development of methods and instruments for testing materials, devices, and structures; advisory services to government agencies on scientific and technical problems; invention and development of devices to serve special needs of the Government; and the development of standard practices, codes, and specifications. The work includes basic and applied research, development, engineering, instrumentation, testing, evaluation, calibration services, and various consultation and information services. Research projects are also performed for other government agencies when the work relates to and supplements the basic program of the Bureau or when the Bureau's unique competence is required. The scope of activities is suggested by the listing of divisions and sections on the inside of the back cover.

\section{Publications}

The results of the Bureau's research are published either in the Bureau's own series of publications or in the journals of professional and scientific societies. The Bureau itself publishes three periodicals available from the Government Printing Office: The Journal of Research, published in four separate sections, presents complete scientific and technical papers; the Technical News Bulletin presents summary and preliminary reports on work in progress; and Basic Radio Propagation Predictions provides data for determining the best frequencies to use for radio communications throughout the world. There are also five series of nonperiodical publications: Monographs, Applied Mathematics Series, Handbooks, Miscellaneous Publications, and Technical Notes.

A complete listing of the Bureau's publications can be found in National Bureau of Standards Circular 460 , Publications of the National Bureau of Standards, 1901 to June 1947 ( $\$ 1.25)$, and the Supplement to National Bureau of Standards Circular 460, July 1947 to June 1957 (\$1.50), and Miscellaneous Publication 240, July 1957 to June 1960 (Includes Titles of Papers Published in Outside Journals 1950 to 1959)( $\$ 2.25$ ); available from the Superintendent of Documents, Government Printing Office, Washington 25, D. C. 


\section{NATIONAL BUREAU OF STANDARDS Eechnical Mote}

157

DECEMBER 31, 1961

\section{INFORMATION SELECTION SYSTEMS RETRIEVING REPLICA COPIES:}

\section{A STATE-OF-THE-ART REPORT}

Thomas C. Bagg and Mary Elizabeth Stevens

NBS Technical Notes are designed to supplement the Bureau's regular publications program. They provide a means for making available scientific data that are of transient or limited interest. Technical Notes may be listed or referred to in the open literature. 


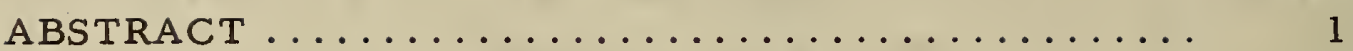

1. INTRODUCTION $\ldots \ldots \ldots \ldots \ldots \ldots \ldots \ldots \ldots \ldots \ldots$

2. HISTORICAL BACKGROUND .............. 9

(Table I. Document Retrieval Devices) ............ 35

3. COMPARATIVE CHARACTERISTICS OF AVAILABLE AND PROPOSED SYSTEMS .............. 37

(Table II. Systems Evaluation Criteria) ........... 39

3. 1 Continuous Rolled Film Systems ........... 40

3. 1. 1 Rapid Selector ................. 40

3.1 .2 FOSDIC II .................. 48

3. 1.3 FileSearch ................... 49

3. 1. 4 Automatic Image Retrieval System ....... 56

3. 1.5 Photo-Magnetic System ............. 57

3.1 .6 Video File.................... 58

3. $1.770 \mathrm{~mm}$ Selector ................. 60

3.2 Chip or Discrete Unit Record Systems ......... 60

3.2. 1 Micro Research System ........... 60

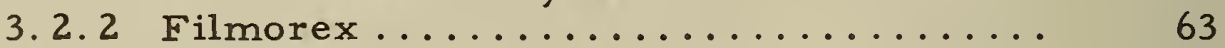

3.2.3 Minicard .................. 68

3.2.4 Magnavue .................. 76

3.2. 5 "Is There a Doctor in the House?" ....... 78

3. 3 Sheet or Strip Film Systems ............ 82

3.3.1 Microcite ................... 83

3.3.2 Large-Capacity Document Storage and
Retrieval System (IBM-Walnut)....... 86

3. 3. 3 Documentary Storage and Retrieval System - Staats ............... 90

3. 4 Microfilm Aperture Card Systems .......... 90

3. 5 Address-Type Selection Systems ........... 95

4. SYSTEM DESIGN CONSIDERATIONS ........... 98

5. THE STATE OF THE ART AND PROSPECTS FOR FURTHER DEVELOPMENTS 
5.1 Storage Media and Equipment............ 103

5.2 Applications..................... 108

5. 3 Implications for Further Development......... 112

6. ACKNOWLEDGMENTS ..................... 115

APPENDIX A - Bibliography................ 117

INDEX .............................. 149

APPENDIX B - Summary Chart.............. 173 
Page

Figure 1. Schematic Diagram of Bush Rapid Selector 20

Figure 2. Film Formats for ERA and Yale Selector 24

Figure 3. Spectral Data Card 29

Figure 4. Rapid Selector Encoder 42

Figure 5. Rapid Selector Film Transport Schematic 44

Figure 6. Rapid Selector $\quad 45$

Figure 7. FOSDIC II Camera 50

Figure 8. FOSDIC II 51

Figure 9. FileSearch Camera 53

Figure 10. FileSearch Selector 54

Figure 11. Micro Research Card 62

Figure 12. Filmorex Chip 64

Figure 13. Filmorex Camera 65

$\begin{array}{ll}\text { Figure 14. Filmorex Selector } & 67\end{array}$

Figure 15. Minicard Document Camera 69

Figure 16. Minicard Selector-Sorter $\quad 70$

$\begin{array}{ll}\text { Figure 17. Minicard Computer Duplicator } & 71\end{array}$

Left: Operator's Console

Middle: Data Processor

Right: Film Card Unit

Figure 18. Minicard Film Processor 72

$\begin{array}{ll}\text { Figure 19. A Minicard } & 74\end{array}$

Figure 20. Magnavue Display Unit 79

Figure 21. Data Bank Format 81

Figure 22. Microcite 84

Figure 23. Microfilm to Film Strip Conversion System 88 (Walnut)

Figure 24. Document Image File (Walnut) 89

Figure 25. Staats -- Document Storage and Retrieval System 91 


\section{INFORMATION SELECTION SYSTEMS}

RETRIEVING REPLICA COPIES:

A State-of-the-Art Report

Thomas C. Bagg and Mary Elizabeth Stevens

\section{ABSTRACT}

A state-of-the art survey on information selection systems that retrieve replica copies of selected items as the products of search has been conducted by the Research Information Center and Advisory Service on Information Processing, National Bureau of Standards, under the sponsorship of the Council on Library Resources, Inc. After discussion of the general historical background of the development of such devices, 15 specific systems employing search-type selection principles are described. In addition, microfilm aperture card systems and related devices as used for addresslocation retrieval are briefly discussed. In general, it is concluded that many advantages of compressed data storage, integral indexing, mechanical selection, and graphic facsimile-copy output can be achieved through use of currently available technology. The principal problems remain those of initial human analysis of document content, of effective manmachine communication, and of determination of realistic user requirements. A bibliography of several hundred literature references pertinent to the survey is included.

\section{INTRODUCTION}

For over a quarter of a century, it has been recognized that mechanical tools to aid in the handling of scientific information must be found. This is because the exponential growth of the technical literature poses unprecedented problems both of storage and of search. In recent years, more and more individuals and many different organizations have become aware of new possibilities of mechanization in the solution of specific storage, selection, and retrieval problems. Attention has been directed to new techniques and media for information storage in compact,readily retrievable form. These new developments may be combined with means for 
the identification of information items so that those desired may be automatically selected from a store or file.

This report discusses the development and current status of a group of information storage and retrieval devices that fulfill the following requirements (assuming the prior establishment of a suitable system index):

1. Storage of a large amount of documentary material in microform

2. Storage of coded index information physically adjacent to or mechanically linked to the stored document image,

3. Mechanical search of this coded information, and

4. Reproduction of a facsimile of the original document(s) selected as a result of the search.

Practical progress has been made in the use of various mechanized devices as applied to particular small areas of this field. In other cases, mechanized tools have been applied prematurely and with insufficient attention to practical usage requirements and to the fundamental, but formidable, intellectual problems involved. Research and development efforts have also been directed to long-range objectives for improved utilization of scientific information in a very general context. In this case, there is often an assumption that new technological developments will be required before a proposed program could be put into effect.

This is usually caused by failure to appreciate the possible applications of currently available technology. The practical realization of these applications would of course require that the necessary systems analysis has been carried out, resulting in a plan for a practical and economical balance of man-machine efforts in the total system. If the result of such an analysis does not indicate precise performance specifications with respect to user requirements as well as to equipment characteristics, the application is not likely to be successful. The difficulties will be related less to the equipment than to the lack of proper preparation for the machine, particularly, failure to plan adequately for document analysis, indexing, and machine coding.

The possibilities for premature attempts at application are often combined with a lack of necessary emphasis on total system requirements. In this sense, a state-of-the-art report on one or more classes of currently available mechanical aids is itself premature. On the other hand, currently available tools may, indeed, find useful application for specific situations. Knowledge of what is already available should help to direct and to motivate the essential systems planning and systems analysis required for successful application. 
The purpose of the present report is to review certain aspects of currently available mechanized techniques for storage, selection, and retrieval with the objectives just discussed in mind. It must be pointed out that the simple devices for mechanized information retrieval and the special-purpose systems which have been successfully used to date are essentially mechanized aids to people. It appears that this is the way even the most complex systems and equipment must be used.

In general, there is agreement in the field today that there is a need for automatic processing techniques and for mechanized aids at all levels of information storage, search, selection, and retrieval. There is increasing awareness of need for mechanized document retrieval devices. When such machines or devices are to be considered, the following two major factors must be kept in mind:

1. The specific application for which the equipment is to be used, especially the needs of those whose operations are to be aided; and

2. The means of communication between man and machine.

This report is based upon the results of a survey conducted by the Research Information Center and Advisory Service on Information Processing (RICASIP), National Bureau of Standards, under the sponsorship of the Council on Library Resources. It is a summary report which will review the characteristics of a particular group of document retrieval devices. The devices described are those which search some form of a coded file that is associated with a graphic representation of the filed information. In particular, this report will examine some of the factors that determine the latitude currently available to the user in setting up an information selection and retrieval system where graphic-facsimile copies of the selected items are desired as direct outputs. A prime system requirement for graphic-facsimile or replica copies to be retrieved as the result of search is, of course, the use of a storage medium which will permit ready reproducibility of the replicas.

In large files and libraries there are obvious advantages to be gained by providing copies as selection-retrieval output products rather than the original items themselves. Simultaneous access by different users is thus assured, and the integrity or completeness of the collection is maintained at all times. The information that may be stored in such systems is relatively unrestricted as to content. It can include complete documents, abstracts of the literature, technical drawings and blueprints, maps, aerial or microstructure photographs, and, in particular, items containing admixtures of textual and pictorial information.

A second system requirement, especially for large collections of recorded knowledge, may be for compact storage. In such cases, 
the need for compressed information storage as an operational requirement leads to increasing use of various microforms. 1/ Then, as data reduction ratios increase, the second major factor, that is, the means of communication between man and machine, takes on additional importance. As microphotographic techniques are applied, the small size of the recorded items and the difficulties of their manipulation by the human combine to restrict the feasibility of manual scanning of the stored text as a method for selection and retrieving. Retrievability may therefore depend on the use of marks, holes, or other encoded indicia for subsequent selection. These are imposed on or added to the stored item itself.

In many retrieval operations, the address of a stored item in the file is known, or other locator information, such as the serial number, the author's name, and/or the title of the paper, can be used directly in a straightforward look-up operation. The dictionary, telephone directory, and the file alphabetically arranged by names of correspondents are obvious examples of efficient and easy ways in which address file systems may be used. Further, if the items in the file are to be stored as microforms, the addresses and locatorclues may be encoded and attached to the items in machine-usable form so that mechanized retrieval methods may be applied. Thus, for very large document collections a variety of mechanical handling devices is being developed to retrieve documents in this manner. In general, however, this report is concerned with such equipment only in so far as it may be used as an adjunct to more versatile searching systems.

We distinguish, therefore, between document retrieval devices that use address-type selection and those that use search-type selection. The distinction is a direct result of the basic differentiation between two unlike situations. The first is the case wherein the address of the desired file information is known in advance and the information is retrieved on the basis of its location within the file. The second, which includes both the file reference approach and the search prescription approach, involves the use of search terms to locate the data according to its contents as indexed. In this second case, a specific address is unimportant because the document or data not only can but usually will be identifiable in a variety of ways. In

Heilprin, L. B. , "Communication engineering approach to microforms," Ref. 122, p. 213: "Microform is a general word which includes microfilm, microfiche, film strips, insert cards, and the like - any opaque or transparent carrier of a miniaturized permanent message." 
fact, it is not known in advance whether one, many, or no items of information will match the searcher's request to the system file.

In the file reference approach, the information to be processed is manipulated, counted, sorted, combined in various ways, stored or filed, and the datum itself is usually retrieved, such as the value for the melting point of molybdenum as recorded in a file of test reports. In the search prescription approach, on the other hand, the information which is directly processed usually consists of information about other information, such as the indexing terms or other "retrieval hooks" used to describe the subject contents of documents in a technical literature collection.

Both the file reference and the search prescription approaches may involve searches of a collection of stored items in order to locate and retrieve individual items which meet the selection criteria specified by the potential user. If the purposes of the typical user in requesting a search are to locate and recover a specific fact or a particular paper by a particular author, the file reference system may best meet the specific application requirements. On the other hand, if the purpose is to review the pertinent literature in order to plan new work, to locate suggestions for carrying out an experiment, or to ascertain whether or not particular results have been previously reported, some form of search prescription will be required.

The distinction between the two approaches is also evident with respect to the factor of man-machine communication, especially in terms of the means available to identify the material for machine processing. In the file reference case, the problem of describing to a machine what information is desired is typically that of specifying the location by address-in-storage of an item to be retrieved. Alternatives are to indicate the title, name of author, and other bibliographic identification or similar "look-up" cues. In the search prescription case, conversely, the problem typically involves first a determination of the possible terms under which a relevant item might have been indexed or classified and secondly, a search of appropriate records for items so indexed.

In general, the distinction between the file reference and search prescription approach can be further emphasized by recognizing that for most file reference applications existing equipment and welldeveloped data processing techniques can be and are being successfully used. The situation with respect to the search prescription approach, however, presents added problems. This is true both with respect to possibilities for mechanization and with respect to the even more difficult and challenging area of how to identify and categorize the documents for items that are to be stored and subsequently searched. It is in this latter area that the problem of developing effective communication between man and machine is particularly acute. 
Similar considerations both as to the distinction between the two approaches and as to the difficulties inherent in the search prescription type of problem have been covered in a statement by Dr. Alan T. Waterman to the Senate Committee on Government Operations. This was in connection with a general study of the problems of documentation of scientific information. Dr. Waterman noted three types of information storage and retrieval operations. The first type concerns the manipulation of numerical data and names and characteristics of individuals and organizations, and involves the use of straightforward data processing techniques. The second type, which involves the printing of indexes and bibliographies, has found success in the practical combination and application of existing equipment and techniques. However, with respect to the third or search prescription type of operation, Dr. Waterman had the following to say:

"The third type of mechanization is much more difficult and is concerned with the processing of the subject content of scientific and technical documents for storage and automatic searching. In this area of mechanization we and other agencies support a good measure of research to determine how best to identify and to code the significant information in scientific documents and whether these steps, as well as searching procedures, can be mechanized in order to handle large collections of information effectively....

"One of the difficulties of mechanization of storage and search operations.... is that of the effect of volume of material to be handled in the process. Procedures that work well for a few thousand or tens of thousands of documents probably will not adequately handle the volume of material that would have to be processed for a major scientific discipline, such as chemistry, for example..... The relationship of volume to the complexity may be stated as the larger the volume of material that is to be handled by a retrieval system to serve scientists, the more detailed must be the analysis and indexing of the subject content of the documents. This must be so in order to provide the highly selective discrimination that will make it possible to avoid swamping the inquirer with too many documents of tenuous relevance to his question. "1/

In considering machines as aids to the solution of search type selection problems, then, we note first that the major difficulties do not lie in the hardware but rather in the means for identifying the documents in terms of subject content, i.e., the difficulties of

1/ Senate Document No. 113, Ref. 284, p. 95. 
man-machine communication. 1/ Again, it should be made clear that any machine serves primarily as an aid to the user. The use of machines therefore presents the communication problem both in the input operations, e.g., the translation of indexing information into machine-usable language, and in the search prescription process. In the latter operation, the critical problem is for the questioner to frame his request for desired information in language appropriate to machine search.

Organization of information items that are to be stored so that subsequent search may be expedited involves the identification of each item in terms of one or more criteria or retrieval hooks which, it is assumed, will be useful for subsequent selection. The choice of criteria assumed to be useful reflects the requirements of the specific application. For file reference situations, the criteria might include, as we have seen, accession number, name of author or is suing agency, date of publication, and the like. For search prescription systems, however, the selection criteria which are used would typically represent the results of human analysis, classification, and subject-content categorization of the probable meanings of the messages conveyed by the item. Selection by a title or permuted title exists as a transition area between the file reference and search prescription situation.

The process of organizing items for search, preparatory to storage, thus covers the broad area in which both the traditional library classification and the newer, less conventional, indexing techniques are applied. This process is therefore one in which critical problems of language, of meaning, and of meaning-ofmeaning (or "aboutness") are encountered. It is for this reason that the efficient organization of items, prior to storage and for later search, constitutes "an intellectual problem of formidable proportions. "니

For this reason, a comparative evaluation of different techniques of mechanization, different devices, and different storage media is relatively meaningless unless the same collection is subjected to the

1/ Herner, S., "The relationship of information-use studies and the design of information storage and retrieval systems", Ref. 124;

Manly, R., "The inadequacy of varying the depth of indexing and other 'document collection' approaches to information retrieval for researchers", Ref. 171 .

2/ Bush, V., "Report to the Secretary of Commerce by the advisory committee on application of machines to Patent Office operations", Ref. 46, p. 8. 
same depth of content analysis and is searched for the same user purposes. A combination of specific, user-centered, operational requirements and of man-machine factors predominates in the success or failure of a particular mechanized technique or tool in the overall selection and retrieval system. If we accept a definition of storage, selection, and retrieval efficiency as being "maximum transfer of information of high quality, within the least space, at the lowest cost," 1 /we must recognize that the overall costs include not only the human effort necessary to reduce the subject-content analysis to machine usable form, but also the human effort necessary to sample, appraise, and make effective use of the search products.

It is these human factors, rather than the technical factors, that form the present frontier of information selection and retrieval. The question of the kind and depth of subject content analysis and indexing permitted in a system is in a general sense outside the scope of a state-of-the-art study of specific storage media and selectionretrieval equipment. However, the interplay among such factors as depth of subject content analysis, choice of coding system, capacity for accommodating coded representations of the selection criteria, and ability to provide an open-ended system that permits up-dating and reorganization on the basis of actual use should certainly be examined.

To summarize, then, we shall assume first that a suitable indexing system has been established. Secondly, we shall limit detailed descriptions to those available techniques in which the selection and retrieval devices are designed to provide graphic replica copies of the documents that are to be selected and retrieved. We shall emphasize the search-type selection systems rather than those which are mechanized aids to retrieval by means of bibliographic citations, accession or serial numbers, or other direct address-clues. Finally, we shall be particularly concerned with devices that provide for the physically adjacent combination of the selection criteria information and the stored material itself. This is sometimes termed integral indexing and provides for different degrees and/or depths of representation (coding) of the subject content of documents stored. Thus, a single selection-retrieval system will provide different levels of access and, hence, permit flexibility without compromising the direct graphic-replica output feature.

1/ Heilprin, L. B., "Communication engineering approach to microforms"., Ref. 122, p. 214. 


\section{HISTORICAL BACKGROUND}

During the past three decades, a number of devices have been proposed which are based upon the recording of actual documents in reproducible form, e.g., on various photographic media, and which provide for coded information (combined with the recording of the item itself) to serve as the means for identification and selection of items to be retrieved. This system design approach permits the direct retrieval of a full replica copy. Such replica copies are graphically identical to the original filed items that are selected by virtue of meeting the criteria enumerated in a specific search prescription.

The devices proposed represent various combinations of storage media and processing methods, and they were designed for several different applications. In the devices considered in this report, the combination typically consists of the following: (1) storage media providing for ready reproduction of the information or images stored, (2) compressed information storage, (3) use of the principle of integral indexing, and (4) means for machine manipulation of the file in the operations of search, selection and retrieval.

Of these, the use of the principle of integral indexing in documentation operations has by far the longest history. In ancient Sumaria it was sometimes the practice to cover a cuneiforminscribed clay tablet with a thin clay layer. This covering layer carried access clues to the more detailed information recorded on the tablet itself. 1/ The stamping of the Dewey Decimal or Library of Congress classification code on the spine of a book has been library practice for many years. A modern example which combines compressed data storage with integral indexing is the so-called pocket card used for various literature search files. Typical of its use is the patent file at the Cables and Insulating Materials Research Institute in Czechoslovakia. The pocket card itself carries information as to the UDC classification code, the first application

1/ Compare, for example, Lalley, J. M., "A treasure lost in unread wedges", Ref. 157, p. A-10: "The characters were impressed on tablets of soft clay by the triangular end of a blunt stylus, hence 'cuneiform', or wedge-shaped. Then the record was made permanent by baking the tablet. Sometimes the tablet was covered by a thin envelope of clay, and baked again. The envelope could be broken and removed without difficulty or damage; it might also be inscribed with a name and address and a brief description of the contents - very useful no doubt for delivery and for purposes of filing. " 
date, the patent class, and the patent number. It contains both photo-copies of abstracts as published in abstracting journals and a microfilm copy of the patent specification itself.

Other modern examples of retrieval systems in which the original item itself is retrieved on the basis of integral index code include two separate systems developed at the Edwal Laboratories in Ringwood, Illinois. $2 /$ The first is for the indexing of photographic slides by punching holes at dedicated positions along the edges of the slide itself. However, only 10 such positions are used, and a separate master index code must be consulted in order to identify the "address" by which desired slides may be located. $3 /$ The second example is a system devised by Thomas T. Hill of the Edwal Laboratories for philatelists. It uses $5^{\prime \prime} \times 8^{\prime \prime}$ McBee cards and has been described briefly as follows:

"From one to ten stamps can be mounted on the face of a card with the name of the country and the date typed on the card. Technical and descriptive information is coded and the file can be hand-sorted for information on such things as perforation method, type of ink and paper, subject, historical connection and type of stamp." 4 /

Means for machine manipulation of items in a file for the purposes of selecting and retrieving certain specific items date from at least the development of punched card machine tabulation equipment. As developed by Hollerith in the $1890^{\prime} \mathrm{s}, 5$

Cigánik, M.', "Scientific, technical, and economic information in a research organization, "Ref. 54, pp. 632-635. Similar techniques are used elsewhere, for example, in the British Patent Office.

2/ Casey, R.S., J.W. Perry, M. Berry and A. Kent, "Punched cards - - their application to science and industry", Ref. 50, pp. 333-334.

Patton, A. R., "Punch card filing system for your slides", Ref. 211 .

4/ Haksteen, B. L., "Review of applications", Ref. 113, p. 333.

Martin T.C., "Counting a nation by electricity", Ref. 172, and Rex, F.S., "Herman Hollerith", Ref. 242. The principles of recording and tabulating by machine had been sufficiently well established by 1890 that punched card processing was actually used for the 1lth Decennial census. Hollerith's first patent for such equipment was is sued on January 8, 1889 . 
card carries information coded as a pattern of holes. When the same or other information is also imprinted on the same card in humanly legible form and when the card is selected on the basis of the pattern, a special case of integral indexing combined with mechanical manipulation is achieved. Further, these advantages may also be combined with those of a storage medium which provides for compressed data storage as in the use of microfilm inserts in aperture cards.

Compressed data storage, especially by use of photographic techniques, antedates by at least several decades the development of punched cards and of equipment for the manipulation of discrete items carrying recorded information. The date of 1839 marks the first known instance of microphotography. In that year, John Benjamin Dancer of Manchester, England, installed a microscope lens in a camera and succeeded in making a microphotograph of a document. I/Rene Dagron, however, is generally credited with being the inventor of commercial microfilm. The first microfilm patent ever granted was issued to Dagron in France in the year 1859. He also may be credited with personal supervision of the first use of microfilm other than as a novelty, that is, for the sending of dispatches by carrier pigeon during the siege of Paris in 1870. $2 /$

Thus, as noted by Watson Davis in a plea for microphotography in the service of scientific information in 1936, "microphotographic duplication, both as an idea, and practically, is not new. "3/ Actually, the potentialities of microphotography for compressed data storage with particular reference to books, records, library catalogs, archives, and libraries have been repeatedly suggested by

1/ Luther, Frederic, "Microfilm - a history - "1839-1900", Ref. 164, p. 16: "This, the earliest example of microphotography on record, had for its subject a document twenty inches long; at the 160:1 reduction used by Dancer the image was one-eighth of an inch in length, yet the writing on the document was described as perfectly legible under a $100 \mathrm{X}$ microscope."

2/ Ibid., pp. 58-82.

3 Davis, W. "Microphotographic duplication in the service of science," Ref. 66, p. 402. 
photographers, scientists, and others, from 1851 onward. 1/ Microphotography in the service of documentation, however, probably received its first practical application with the initiation of the Bibliofilm Service in the Department of Agriculture Library in November, 1934 II

During the $1930^{\prime}$ s, then, microfilming of books and records was established as practical. Thereafter, many individuals proposed ways for mechanical handling, sorting, and filing of records maintained in microphotographic form. Punched card equipment and punched paper tape systems for handling business and statistical data were also well developed by that time. Goldberg, Bryce, and others approached the problem of combining photographic records with mechanical manipulation largely from the point of view of accounting, banking, and statistical records processing. Investigators such as Seidell, Draeger, Davis, and others stressed the documentalist approach in terms of new means for improved utilization of the scientific literature.

In 1931, Emanuel Goldberg of Dresden, Germany, was issued U.S. Patent 1,838,389 for a statistical machine. The Goldberg disclosure reads, in part, as follows:

"This invention relates to a method of and means for controlling statistical machines, and its object is to provide a method and means in which counting devices, for example, may be controlled in action by radiating energy such as rays from a selenium cell, so that simplicity and rapidity of action may be served....

"This idea can be realized in various ways. A perforated card can be put in front of a series of selenium cells, lightelectric cells, thermoelectric piles or bolometres (called

For early examples, see the discussions and quotations from the Jury on Photography, 1851 London World's Fair; the American Journal of Photography, 1858; Photographic News, 1859; Sir John Herschel writing with respect to microscopic editions of reference works in 1853, and Sir David Brewster, physicist, witing for the Encyclopedia Brittanica in 1857. (Ref. 164, passim)

2/ Davis, W. "Microphotographic duplication in the service of science," Ref. 66, p. 402-403. It is to be noted that the combined interests of F. G. Cottrell, E. E. Slosson, and W. Davis had resulted in suggestions for microphotographic duplication services applied to scientific literature as early as 1925. 
hereafter 'radiation indicators'). Only the instruments receiving the rays of radiating energy through a perforation will be influenced, all others remaining immobile. A spot translucent for the kind of radiation in question can be used of course in place of the perforation. This is a particularly great advantage for the process, as thereby the perforated plates can now be substituted by plates made by photography.

"This development of the process is of great importance if a combination of different statistical records is desired. In place of the perforations a positive photographic transparency... provided with black letters, words, figures or other recording marks can then be used, further a negative... of a certain combination of figures or words (called hereafter 'searchplate') is made. If the transparency containing the various statistical indications is now run through the machine in such a manner that the negative coincides with the transparency a complete coincidence impenetrable to any light or heat radiation will only be possible in one defined case; this will only occur when the negative bears exactly the same characters, marks, figures, etc., as the transparency in question, the only difference being that in the negative these records are light on dark ground, while in the transparency they are dark on light ground. A certain combination has thus been picked out of a large number of others with extraordinary speed and reliability hitherto not attainable....

"Another feature.... is the fact that the instrument indicating the existence of a certain statistical combination serves to simultaneously record the statistical indications connected with the combination. The light falling through the transparency can for instance be divided into two parts.... One part falls on the negative containing the combination desired (search-plate), and the other on a sensitive photographic plate.... protected by a shutter .... connected with the photoelectric cell. Thus the access of light to the photographic plate is possible when the radiation indicator records the existence of the combination desired or looked for. By this method certain defined cases out of a large number of statistical indications are picked out and recorded on the photographic plate...." 1 /

Thus, as early as 1927, when Goldberg filed a patent application for this device in Germany, there was a proposal for a system combining principles that are basic to many of today's selectionretrieval systems, as listed below:

1/ Goldberg, E., "Statistical machine," U. S. Patent No. $1,838,389$, Ref. 105. 
1. Use of photographic storage media for records and items to be subsequently selected and retrieved.

2. Use of integral indexing in that the selection criteria are physically recorded on the same storage medium as the information items to be selected.

3. Means for mechanical manipulation and search, in this case including a search plate or search mask which carries the photographic negative image or a similar complement of the specific selection criteria to be used for selection and retrieval.

4. Means for providing replica copies of information identified as being desired by coincidence of the search plate indications with the recorded selection criteria, in this case by a photographic copying process triggered by extinction of light to a photocell.

Similar but independent suggestions for information selection systems using photographic storage and providing replica copies were proposed by several different investigators during the decade or so following Goldberg's patent. James W. Bryce was issued a number of patents dealing with yarious equipment developments applicable for such systems. 1 For example, in Bryce's U.S. Patent 2, 172, 330, he claims the following:

"A film printing machine including a master film scanning station with means for scanning the indicia disposed on the master film, said scanning means comprising a source of light rays and light responsive means controlled by the light rays modified by the indicia, a circuit network including means for conditioning the network selectively for representing selected predetermined indicia, means controlled by the scanning means for comparing the scanned indicia and the said indicia retained by the conditioned circuit network for determining the relationship therebetween, a detail film, and means for transferring the scanned indicia to the detail film upon determination of a predetermined relationship between the
scanned and predetermined indicia by the comparing means. " $2 /$

1/ Refs. 35, 36, 37, 38 and 39 .

2/ Bryce, James W. "Selective film printer," U. S. Patent No. 2, 172, 330, Ref. 37. 
Bryce, Goldberg, Townsend $1 /$ and Goodale $2 /$, among others, were concerned primarily with integrally indexed storage and selectionretrieval equipment for accounting and statistical purposes. However, it was in this same period of the 1930's that Vannevar Bush initiated the developments which combine equipment techniques of this type with specific documentation purposes, leading to a working model of the Microfilm Rapid Selector.

This early work by Bush was on a document retrieval device that utilized microfilm with mechanical manipulation and search, and provided replica copies. His combination of microphotographic and mechanical search techniques for handling scientific information followed suggestions made by Slosson, Davis, Draeger, Seidell, and others. As early as 1926, Edwin E. Slosson and Watson Davis had urged in correspondence and in presentations of the Science Service organization that attention be given to the use of microphotographic techniques in scientific publication activities. $3 / 4 /$ Stemming from this interest, new possibilities for combining microphotography with mechanized selection-retrieval began to be recognized.

Atherton Seidell, writing in Science in the summer of 1934, first noted the earlier Watson Davis proposals to provide for publication of scientific information in microphotographic form. Seidell then proposed what is probably the first version of microfilm aperture cards, as follows:

"It is probable that the most satisfactory system will be one in which $16 \mathrm{~mm}$ film is used, and from such strips of negatives of printed pages positives will be prepared and these mounted in windows on filing cards. Such cards need not be much larger than $10 \times 15$ centimeters and have sufficient space for typewriting the author's name, title of paper and its classification number..." $\underline{5}$

It is to be noted, of course, that the integral indexing proposed is both of the address type (author, title) and the search type (library

1/ Ref. 279, 280, and 281 .

2/ Ref. 106.

3/ Davis, W., "Scientific publication and bibliography," Ref. 69.

4/ Remarks by W. Davis at the National Microfilm Association's Banquet, 1959, Ref. 68, pp. 76-81.

5/ Seidell, A. "Reforms in chemical publication (documentation)", Ref. 253, P. 71. See also Ref. 254, p. 184. 
classification data). Since the selection material would be typewritten, the actual manipulation and search of the carrier cards would presumably be manual.

However, Davis himself in the following year (1935) noted that the selection criteria need not be limited to humanly legible, humanly manipulatable form. At a meeting of the Institute International de Documentation in Copenhagen, September, 1935, Davis proposed that:

"For every published article, past or current, there would be as many abstract bibliography cards in a master bibliographic file as there are subject classifications under which the article should be indexed. A complete and comprehensive scheme of numerical classification by subject would be created through the utilization of existing subject classifications in science, in so far as they can be utilized (International Dewey Classification?) and the creation of new subject classification schemes for unclassified areas of science toward the creation of which the best scientific and library brains should be directed. Upon each card (the word card is used although the item in the bibliographical index might not be of paper material) there would appear the abstract bibliographical entry and also a subject classification punched or otherwise marked so as to actuate the proper mechanism in a sorting machine of the punch card, photoelectric cell or some other type. Once these bibliographical cards are placed in the files, this sorting machine would file them and select from them the classifications desired. The sorting machine could be linked mechanically to a duplicating machine of some sort (microphotographic camera as used in publication project). In this way it would be possible to receive a request from a scientist for a bibliography upon a certain subject and supply it by setting the sorting machine's selectors to select the number corresponding to that subject classification, the duplicating machine wedded to the sorting machine producing to order the bibliography desired. "I

In addition to the combination of photographic copying with mechanical selection there are two other significant points in the above quotation. These are (1) the idea of multiple copies for cross-referencing (a principle applied, for example, in some Filmorex and Minicard systems), and (2) the important point that the problem of intellectual effort in applying or developing a subject classification must be distinctly separated from the problem of implementing storage, selection, and retrieval.

1/ Davis, W. "Scientific publication and bibliography", Ref. 69 , p. 16, underlining supplied. 
Also in 1935, Rupert H. Draeger (who was then associated with Davis in the Documentation Institute of Science Service) proposed that abstract cards, together with patterns of holes punched into the cards to represent the subject classifications assigned, be photographed onto a microfilm roll for subsequent photoelectric selection. More specifically, Draeger suggested the following:

"Bibliographic abstracts may be made on cards measuring $3^{\prime \prime} \times 8^{\prime \prime}$. The right-hand $3^{\prime \prime} \times 5^{\prime \prime}$ area is used for typing of the bibliographic title and abstract; the left-hand $3^{\prime \prime} \times 3^{\prime \prime}$ space is the selector area, of which the central $2^{\prime \prime} \times 2^{\prime \prime}$ area is used for punched holes the permutations and combinations of which form a pattern for the selector mechanism.

"The cards are prepared... from existing literature, and when sufficient cards have accumulated they are filmed, 32,000 cards to 1,000 feet of film. No definite order of the cards need be maintained.

"The classification of the cards is accomplished on a punching machine operated by the classifier, who sets the machine to the pattern of the classification desired and presses a lever, when the selector area is punched to the desired pattern.

"The selector mechanism projects an image from the selected film in such a manner that the abstract $3^{\prime \prime} \times 5^{\prime \prime}$ area focuses to the plane of a $35-\mathrm{mm}$ positive motion film, while the $3^{\prime \prime} \times 3^{\prime \prime}$ area is diverted by a prism onto the selector pattern. When darkness results due to a matching of the holes, the mechanism is actuated so as to photograph the abstract area onto the $35-\mathrm{mm}$ film.

"A claw mechanism operates the two films synchronously at 10 per second, the selected film moving continuously, while the copy film moves only when selection is made. "1/

Helen M. Davis, also of Science Service, added to the proposals of Watson Davis and Draeger the idea of compartmentalization or file segregation so that abstract cards in the same classifications would be filmed and stored on the same film strip or roll. For automatic searching, the appropriate subject roll would be selected for processing against the specific bibliographic request. She also proposed that the abstract card copies to be supplied to the requester

1/ Draeger, R.H. "A proposed photoelectric selecting mechanism for the sorting of bibliographic abstract entries from $35 \mathrm{~mm}$ film," Ref. 74. 
should contain enough additional address-locator information so copies of the full original item could be ordered if desired. 1 /

It is probable also that Watson Davis and Vannevar Bush had discussed mutual interests in the area of microfilm document retrieval devices as early as 1932. 2 In any case, there are two dominant strands in the tapestry of the historical background of replica-copy retrieval devices. They are those of (1) equipment development with respect to automatic selection and duplication of certain specific information records, and (2) concern for improvements in the use of the scientific literature. In accounting, statistics, and many related data processing operations, it is not usually necessary to obtain a graphic replica copy of the original information item. In the retrospective search of the literature, however, the document or item often contains interspersed equations, drawings, and other pictorial or graphic data which is essential to the understanding of the total text. The two strands of selection equipment and concern for improved retrieval of scientific information were brought together in an actual device in the case of the Bush Microfilm Rapid Selector.

By February of 1938, Bush had formalized his ideas for a document retrieval device to the extent of proposing a specific research project. The work was proposed to be carried out at the Massachusetts Institute of Technology under the auspices of the Research Corporation, which in turn was under sponsorship for this particular work from Eastman Kodak and the National Cash Register Company. The research was to be divided into three stages, as follows:

1. Construction of experimental equipment to test the feasibility of a device which would search reels of coded microfilm at high speed and which would copy selected frames on the fly, for printout and use.

2. Investigation of the practical utility of such equipment by experimental use in a library.

3. Further development aimed at exploration of the possibilities for introducing such equipment into libraries generally.

1/

Davis, Helen M. "Proposal of bibliographic department of the documentary Institute of Science Service," Ref. 64.

2/ Information and copies of pertinent unpublished documents of Science Service have been made available through the courtesy of Watson Davis and Calvin N. Mooers. 
The first Rapid Selector 1 /was constructed at M. I. T. during the period 1938-1940 by Bush and his assistants 2 /. Figure 1 is a schematic diagram of the original Bush Rapid Selector. In this original version, the microfilmed file consisted of document frames accompanied by specific patterns of clear dots used as a selection code. The code pattern consisted of 12 fields, each of which contained 27 discrete indexing areas. Each area represented a different indexing character. This pattern could be used to identify each document by serial number, name or title, author, or subject content indicia. In this machine, the code appeared on one longitudinal half of a $35 \mathrm{~mm}$ film and a photographic image of the original document was recorded on the other half, as shown in Figure 1.

In searching, the code pattern for each document frame was projected onto a bank of photocells. The photocells were masked by a cutout that was the exact physical image of the selection criterion code pattern. Recognition would therefore occur whenever match resulted in all photocells being illuminated. Such a match fired a strobotron that projected the selected frame (document-record half) onto a section of hitherto unexposed $16 \mathrm{~mm}$ microfilm advanced to proper position, thereby allowing a replica recording to be made. Thus, the result of finding a desired documentary item was to copy it when it was appropriately positioned in the selection-mechanism. This preliminary Bush Rapid Selector was used experimentally by the U.S. Department of the Navy during the early years of World War II and subsequently was returned to M.I.T., where it is now stored.

Following World War II, because of interests of the Office of Technical Services,(OTS), Department of Commerce, in improved techniques in the organization and use of scientific literature, Dr. Bush was asked to make his invention available for public use. Under the direction of John C. Green of OTS, two contracts were placed. One, based on proposed specifications as of August 19473/, was negotiated with Engineering Research Associates for the design and

1/ Howard, J.H. "A brief progress report on the rapid selection research", Ref. 128.

Howard, J.H. "Description of the rapid selector camera", Ref. 129.

Howard, J.H., and L. R. Steinhardt, "General description of the rapid selector and the apparatus for preparing the index film", Ref. 131 .

2/ Project Engineer: John H. Howard; Research Assistants: Russell Coile, John Coombs, Claude Shannon and Lawrence Steinhardt. Coile, R.C. "Scientific aids to documentation", Ref. 55, p. 2 .

$3 /$ "General description and proposed technical specifications for microfilm selector", Ref. 84. 


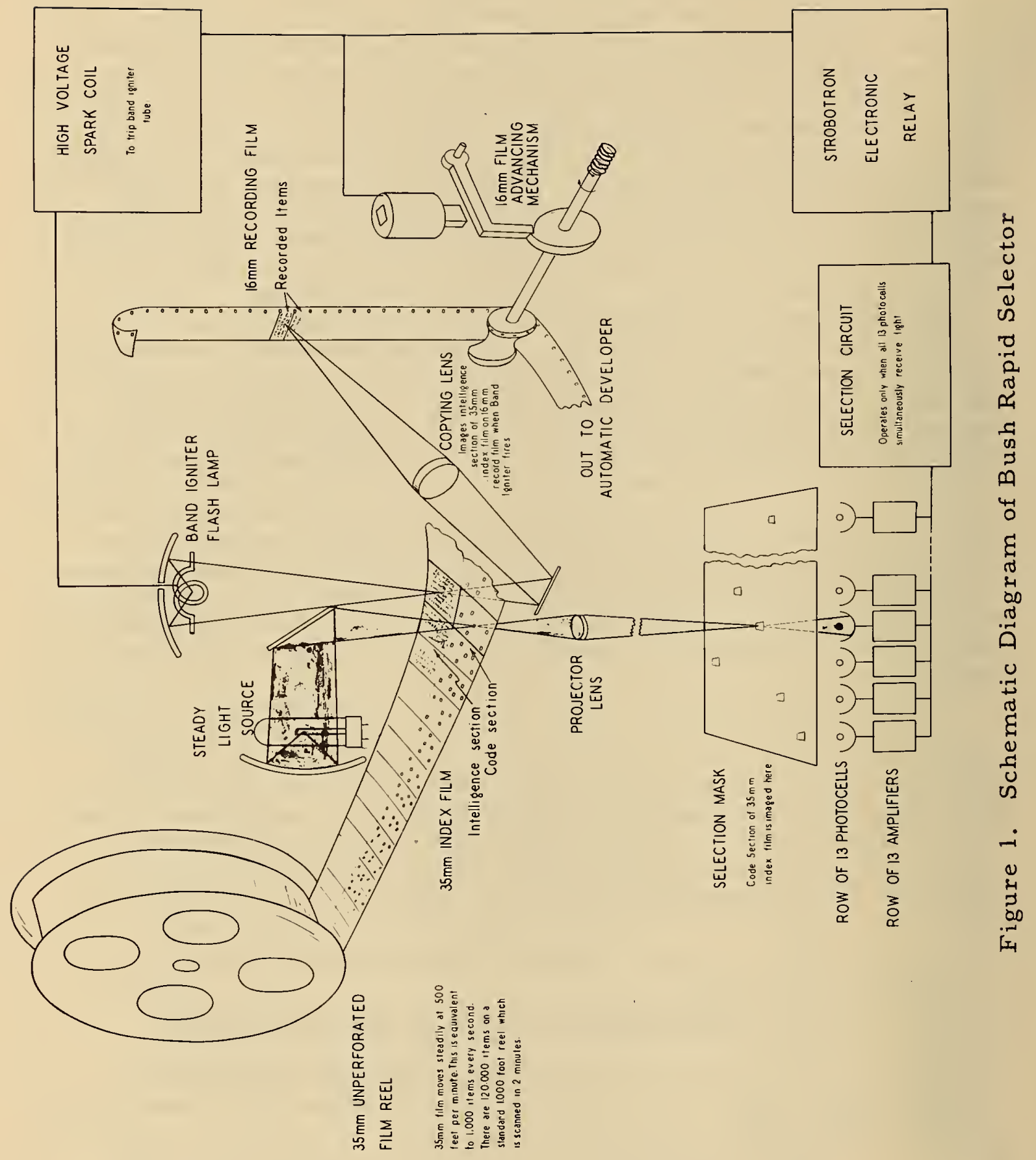


construction of a second model. The other was negotiated with Ralph R. Shaw of the Department of Agriculture Library for the encoding of test material and for practical testing of the device. 1 /

1/ The early history of the Bush Rapid Selector was described by Shaw in 1949, as follows:

"The Rapid Selector is the culmination of many years of development and experimentation, in part directly connected with it, in part in the fields of organization of knowledge, electronics, mechanics, and other fields.

"The first practical application of electronics to selection of data on film appears to have been made some twenty years ago by Dr. E. Goldberg, who at the time was with Zeiss in Dresden. His invention was protected by United States Patent No. 1,838, 389 issued on 29 December 1931.

"The basic principles of organization of knowledge applied in the present machine were developed by Dr. Vannevar Bush at the Massachusetts Institute of Technology more than ten years ago, and while many changes were made in operating details in designing the Rapid Selector, its basic electronic system should be credited to Dr. Bush.

"The funds for development of the Rapid Selector were provided by the Office of Technical Services of the Department of Commerce from an appropriation intended for development of new tools to advance the technological development of the nation and to result in wide public benefit. Mr. John C. Green, Director of the Office of Technical Services, not only supervised the contractual details, but also was of constant assistance in technical aspects of the development of the work.

"The Engineering Research Associates of St. Paul, Minnesota, selected and designed the electrical, optical, and mechanical components, and assembled this first machine.

"The author, with Dr. Bush's permission to use his concept for the public good, developed the programme, devised the method of mounting the coding system so that any size document could be taken without changing the size of the dot codes, devised the triggering dot scheme so that multiple codes could be used in connection with a single reproduction of the text data, and supervised the design, construction, and testing of the machine."

Shaw, R. R., "Machines and the bibliographical problems of the twentieth century"!, Ref. 258, footnote 18 , p. 58 . 
The Rapid Selector built by ERA (principally by John Howard and John Coombs) had code space for seven decimal characters in the form of six selection criterion entries of seven digits each, and could operate with the film running in either direction. The machine used four photocells for character reading. As in the original M.I. T. machine, the second model used a flash lamp to make the output replica copies. Successive document frames could not be copied at high speed because of the time constant requirements in the power supply for the photoflash lamp. To overcome this difficulty, an anticipator was added to slow down the film so that the flash unit would be ready to take the next frame if necessary. 1/

The ERA model was designed to use a 2,000-foot roll of $35 \mathrm{~mm}$ microfilm. On this, up to 72,000 frames of text or abstract material and 432,000 encoded selection entries to this material could be recorded. The searching procedure consisted of inserting a question mask (punched as a negative image) for the desired codes. Searching was intended to proceed at rates of between 70,000 and 120,000 codes per minute. The search run for the entire roll would therefore take between four and six minutes. However, in this model only one code pattern could be searched per run.

In operation, certain mechanical difficulties were encountered with the ERA model of the Rapid Selector. It was at this point that the National Bureau of Standards was asked to assist in overcoming

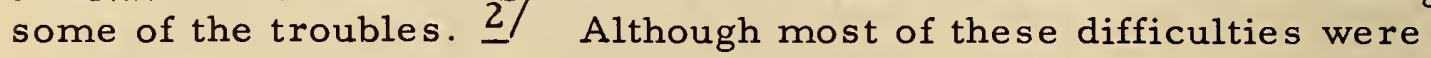

1/ Engineering Research Associates, "Report for the microfilm rapid selector", Ref. 85, and Bagg, T.C. and Greenwald, S., "The rapid selector development program at the National Bureau of Standards", Ref. 14.

2/ Among the difficulties studied by Rabinow and Rotkin at NBS was that of incomplete blackout when the film item code did in fact match the search prescription code. Rabinow recognized that the problems of distinguishing between a true mismatch and an apparent mismatch caused by light leakage or variations in density of recording might be solved by examining the selection code area in small sub-areas one at a time. While not directly used in subsequent Rapid Selector developments, this principle of "best-fit" rather than "extinction" or exact matching has been widely used in automatic character recognition devices. See Rabinow, J. "Optical coincidence devices", Ref. 228. 
minimized, the major factor causing abandonment of the machine was that it was not designed to copy successive frames without delays that severely increased search time. Moreover, the limitation of the selection code area to six selection criteria per document frame and the limitation of the question to one criterion per run had seriously restrictive effects upon indexing and search, and therefore upon the practical use of the selector.

In the early 1950's, a situation arose in which the Central Intelligence Agency desired to explore the potentialities of the Rapid Selector techniques for a large collection of documents where about 4, 800 bits of information had already been used to identify the subject contents. Yale University undertook the re-design of the Rapid Selector to provide for both the greatly enlarged selection code area and successful copying of successive frames without slowing down the search process.1/The design engineers at Yale therefore changed the arrangement of code and document areas. Previously, as we have noted, the film was divided in half longitudinally so that document frames and their corresponding selection code areas were recorded side by side. In this third model, the document image was recorded across the entire width of the film, and the document frame was accompanied by a code area also covering the width of the film, recorded in 40 -bit groups and extending along the film as far as required.

The documents involved had previously been analyzed, and the results had been coded and recorded on punched cards. Five cards' worth of such coded information, representing the subject content analysis of a single document, could be recorded in a code area $11 / 4$ inch long. Thus the code area occupied about the same space as the image of the document at an 8 to 1 reduction. Figure 2 illustrates the recording format for the ERA and the Yale versions of the Rapid Selector. Input equipment used with the Yale machine translated the coded selection criteria as punched in the card to a black or white bit representation. Specifically, the pattern of holes as punched in the card was transformed to a pattern of lighted miniature bulbs that photograph as black dots. This design feature thus made the Selector compatible with standard punched card equipment in terms of the code input operations.

The interrogator system of the Yale model was designed to read the entire code field simultaneously, using one photocell for each bit position in the code field. This arrangement provided considerable latitude in search, especially by comparison with the earlier Rapid Selector models. For instance, any pattern involving any one or more of the 400 columns could be searched. Alternatively, selection could be made on the basis of a range of codes within a column, if desired.

1/ "Nature and use of the Yale Rapid Selector", Ref. 306. 

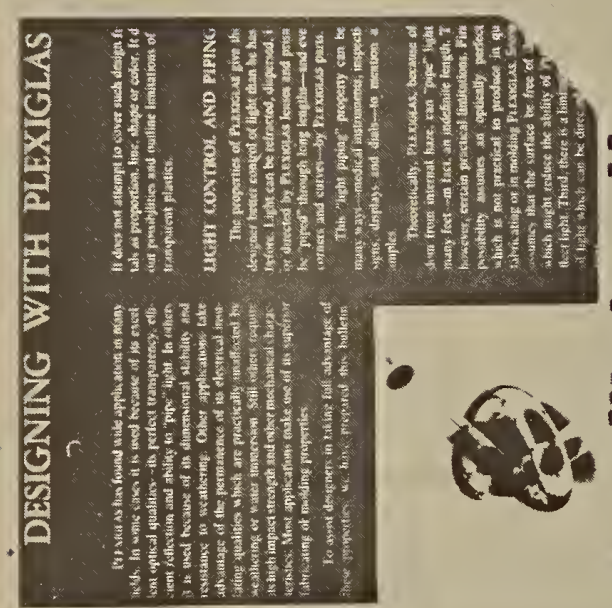

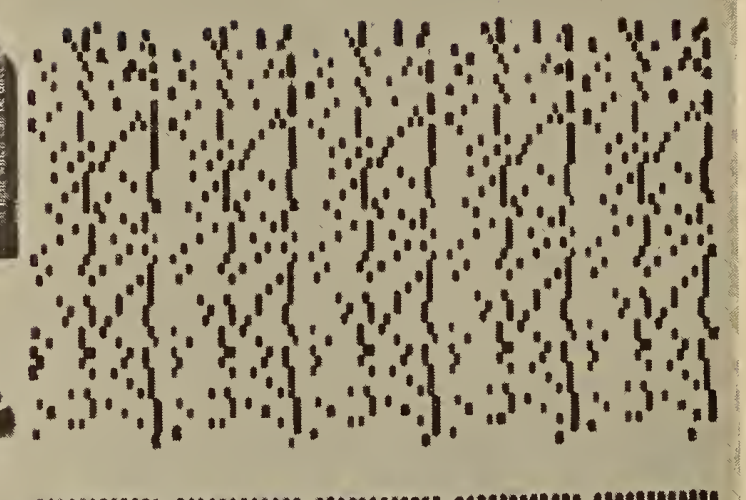

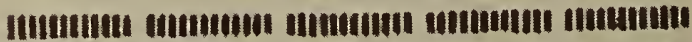

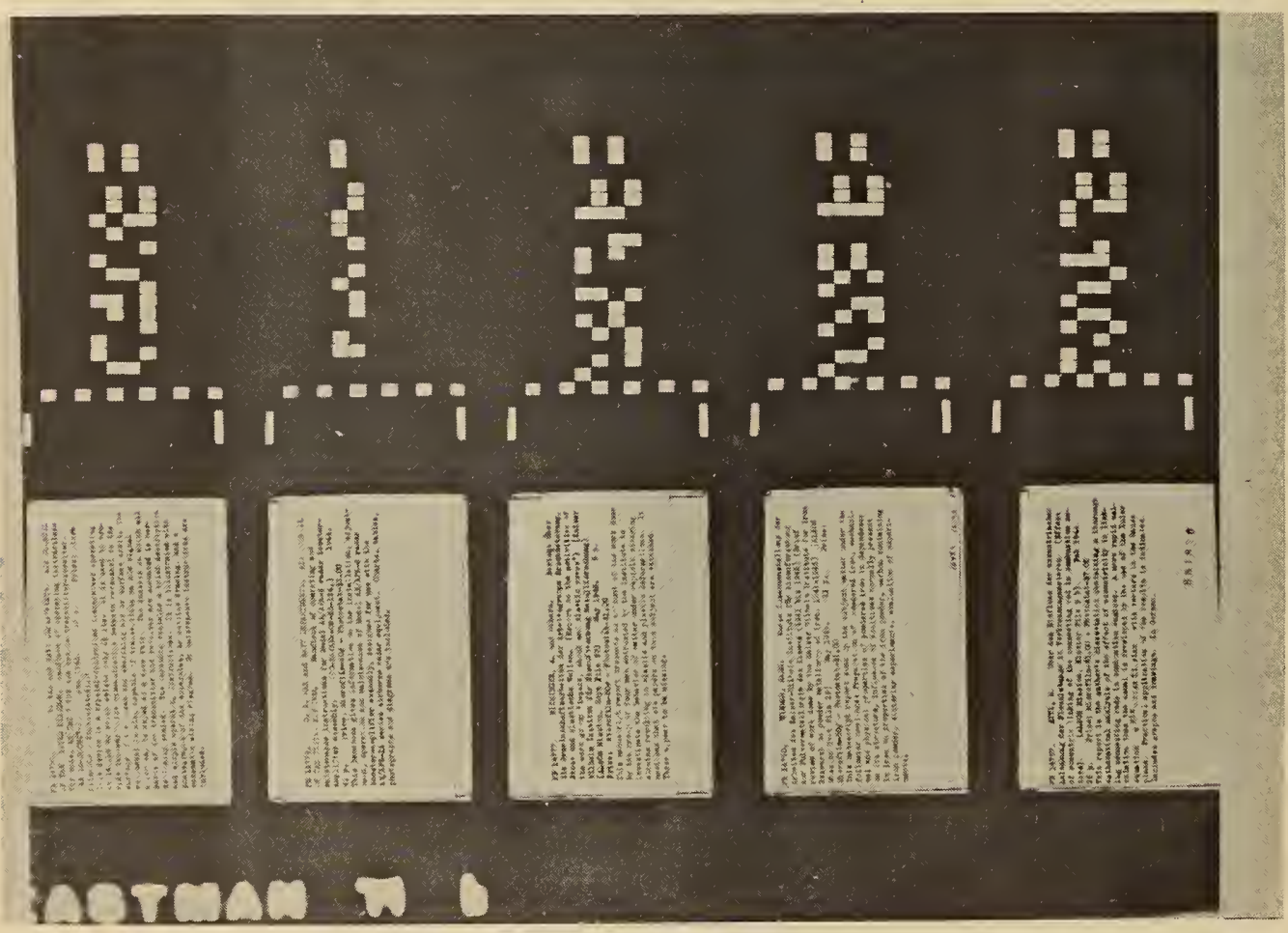


The copying of successive document frames without slow-down was achieved by using a continuously lit conventional projection lamp which illuminated the master film as it passed over a slit. The image was focussed onto another slit behind which unexposed microfilm was placed. The Selector controlled the motion of this unexposed film. When a printout copy of a document frame was called for, the unexposed film was brought up to matching speed so that a 1-to-1 copy could be made. After the copying operation was finished, the film gate was cleared of the newly exposed film, so that a new unexposed section was available for the next print command.

In addition to using this third model as an experimental document retrieval device, Professor Richard Ruggles of the Economics Department at Yale pointed out its potential use for statistical operations. Only digital data, in binary representation, would be used on the film. Under such conditions, and with a film speed of 5 feet per second, the Rapid Selector could read almost a million bits per minute. Unfortunately, the Yale machine was never completed for operational use.

Subsequently, however, the original sponsor, CIA, and Yale University agreed to make this equipment available to the National Bureau of Standards. Further development and experimentation was undertaken at NBS, first in connection with a cooperative NBS-Patent Office program, and later in terms of specific operational applications for the Bureàu of Ships, Department of the Navy. As a result of these efforts, it could be reported as of 1961 that:

"The Rapid Selector - a dream 25 years ago and a laboratory instrument as recently as five years ago - is today the basis of an operating information retrieval system in the Navy Department's Bureau of Ships." $1 /$

Returning to that period of a quarter-century before, however, we note again that there were several different efforts being pursued at about the same time with respect to the Rapid Selector type of device. Two apparently independent but similar patent applications were filed in the years 1937-1938.

The first was filed by Loughridge and Stuart on October 1, 1937, and their invention was patented July 30, 1940. It was for a selective photoprinting system designed to meet the following objectives as described by the inventors:

".... To selectively print enlargements from any frame of a photographic film; to provide each frame of the film with distinctive markings for selective purposes; to

1/ McMurray, J.P., "The Bureau of Ships rapid selector system," Ref. 177. 
provide a control film or band having markings arranged in a predetermined order on the film to register with the markings of the picture film for selective purposes; to provide light sensitive means responsive to the markings of the films when they come into register for controlling the system; to provide a control film or band with predetermined markings which are progressively brought into use for selecting the frames of the film desired; to provide for projecting the picture of the film and the markings of the film through the same gate with light cells responsive to the film markings only and to provide a selectively operated shutter mechanism controlling the projection of the picture but not the light to the light cells. " 1/

Loughridge and Stuart specifically proposed the application of such equipment to collections of photographic records of indices, documents, and books. However, it is clear from the suggested embodiments of the invention that the selection criteria markings were intended to be used as serial numbers distinguishing each frame rather than as direct clues to the contents of the recorded items. This was therefore an address-type rather than a searchtype retrieval system.

The second patent application, filed by R. S. Morse in 1938, 2/ disclosed a search-type system. It covered a combination of the Rapid Selector type principles with the calculating and statistical operations with which Goldberg, Bryce, Goodale, Townsend, and others had been concerned. The possibility of use for "literature abstracts" was mentioned, but the emphasis was upon the recording of "information items" such as identification cards, bank checks, sales records, social security cards, and the like. The film band was to be divided longitudinally with the items along one side and the corresponding codes for selection along the other. Interesting features of the disclosure include the suggestion of sound and magnetic recordings as well as photographic means for the selection code representation. Another feature was the provision for selection on the basis of any four-of-five of the code "tracks", thus providing directly for generic as well as specific searches if the code language permitted. The Morse patent was issued September 8, 1942, and was assigned to the Eastman Kodak Company.

1/

Loughridge, M.H., and Stuart, R. "Selective photographing system", U.S. Patent 2, 209, 342, Ref. 162.

2/ Morse, R.S., U.S. Patent 2, 395, 000, Ref. 190. 
As previously mentioned, Eastman Kodak had also been interested in automatic selection equipment for use with photographic storage media from at least the time of the early Bush proposals. Investigators there did further work on this type of equipment, and as a result designed and built the "Photographic Digital ReaderRecorder," 1/ which was used for a time as input to the Whirlwind computer at M.I. T. Following that, about 1950 Eastman Kodak came to the conclusion that the discrete record approach should be explored for the haydling of very large, rapidly expanding, frequently revised files. This idea ultimately led to the system now known as Minicard. 2 /

A similar approach was taken, apparently independently, by Samain with his Filmorex systems in France which date from at least 1952. 3/ The Minicard, Filmorex, and similar systems manipulate discrete film chips each of which contains a number of code bits recorded in a selection area together with images of one or more document pages.

The particular form of the discrete unit record that utilized a microphotographic recording physically bonded to or inserted in a punched card had been specifically proposed by Seidell in 1934 . 4 / During the early $1940^{\prime}$ 's John Langan and his associates, working originally at the Office of Strategic Services, successfully modified the ordinary machine tabulating card to contain a frame of microfilm. 5 This idea' was pursued commercially by the Film Sort Company (formerly Film'N File, Inc., later the Filmsort Company, a division of Miehle:-Goss-Dexter, Inc., and now a subsidiary of Minnesota Mining and Manufacturing Co.) which called the product "aperture cards".

1/ Eastman Kodak, "The Kodak digital reader-recorder, "Ref. 79.

2/ Tyler, A.W., Myers, W. L., and Knipers, J.W., "The application of the Kodak Minicard system to problems of documentation, "Ref. 282.

3/ Samain, J., "Filmorex," Ref. 249.

4/ Seidell, A. "Reforms in chemical publication (documentation)", Ref. 253, and "The photographic reproduction of documents," Ref. 254.

5/ Langan, J.F., "Record Card," U.S. Patent 2, 512, 106, Ref. 159 At about the same time as the Langan developments, A.V. Loughren was issued U.S. Patent 2, 402, 058 for a "Secrecy Communication System" in which selective scanning of code patterns on a specially designed aperture card would be employed, see Ref. 161 
In 1951, applications of cards with microfilm attachments and inserts had been seriously considered by several of the scientific and industrial contributors to the study of punched cards. $1 /$ For example, C. A. Burkhard suggested:

"In addition to entering numerical data and similar reference information on punched cards it is also possible to attach photographs, drawings, graphs, charts, microfilms, etc., directly to the cards. By sorting for a particular item or topic all corresponding photographs, drawings, and charts may be obtained in addition to the pertinent references and data. This is often helpful in writing papers as it may prove desirable to include photographs, etc., which otherwise might be overlooked." 2

By 1956, aperture cards were used for engineering drawings, real property title records, the "rogues"'gallery of a large metropolitan police department, and copies of labels used on packaged or canned meats sold in the United States. 3 Similar cards that provided for microfilm inserts and integral indexing using the edge $\overline{4}$ / notched-card technique (such as found in McBee's Keysort system)also became available. The use of aperture cards is discussed in more detail in Section 3.4 of this report.

Some variants of the discrete unit record approach are exemplified by the following systems:

1. About 1949 the National Research Council and the National Bureau of Standards sponsored a program to assist in the interchange of infrared spectral data between cooperating laboratories. They proposed the use of a 5" x 8" McBee card (Figure 3) that enabled the selection and retrieval of data about compounds on the

1/ Casey, R.S., and J.W.Perry (Eds.) "Punched cards, their applications to science and industry," Ref. 49. The Filmsort cards and equipment are discussed on pp. 71-74.

2/ Burkhard, C.A. "The use of punched-card techniques in preparing reports, papers, and books", Ref. 41, pp. 183-189.

3/ "205,000 meat labels on file", Ref. 127;

"How MID handles label control", Ref. 126.

4/ Filmsort Division "New horizons with microfilm", Ref. 91, p. 26. 


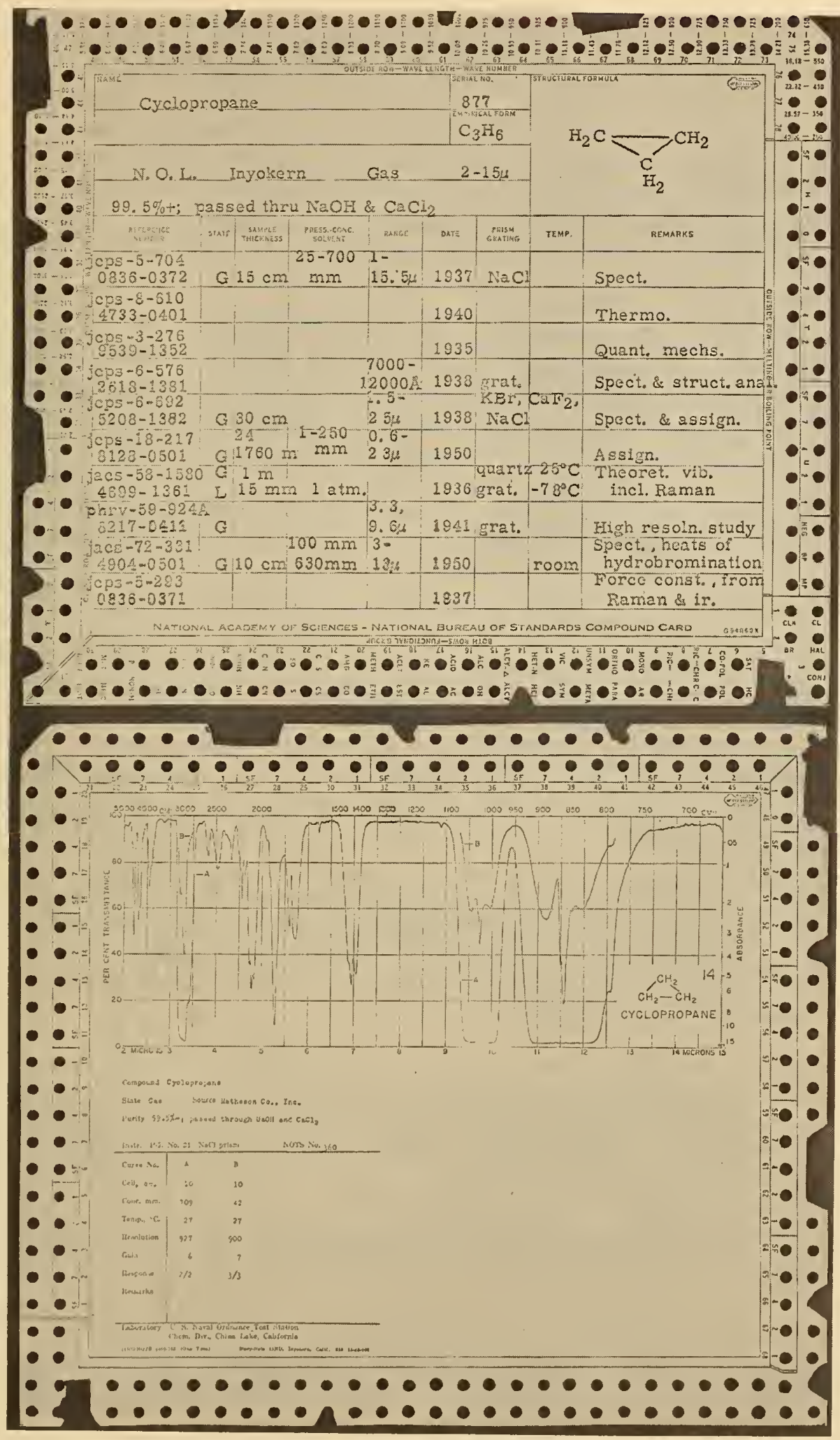

Figure 3. Spectral Data Card 
basis of absorption band position, melting point, molecular functional groups, and number of carbon atoms. In addition, these cards, which were selected by a needle-sorting process, carried a chart tracing of the spectrogram. Such cards were issued by the NBS to subscribers. Similar developments in Great Britain and Germany as well as in the United States led to the Documentation of Molecular Spectroscopy System, 1 y a system now internationally available through Butterworth, Inc.

2. The Recall Film Index System, which existed only as a proposal, was suggested to the Patent Office in 1954. The proposed system would have used Kalfax film, which has an ultraviolet-sensitive heat-developed emulsion that can be processed in normal light. This would provide film cards on which would be recorded both the reduced image of a document and coded index entries or selection criteria. The latter would be produced on the Kalfax film card by contact printing from a punched card. 27

3. The Micro-Research-Cards of the Petroleum Research Corporation are film cards with integral indexing which are actually being used for a collection of documents concerning the petroleum resources, geology, and paleontology of the Rocky Mountain region. As described in 1956, pertinent features of this operational system 3 / were as follows:

"This library contains microphotographic reproductions of approximately 8,000 published and unpublished articles and theses... yet the entire library fits easily into a two-drawer legalsize file cabinet...

"The file contains approximately 8,000 5-by-8 inch film transparencies, each imprinted with up to 100 pages of text reproduced about $1 / 20$ the size of the original. The card is perforated at

1/ "Punched cards facilitate rapid searching for spectrograms", Ref. 227.

2/ "Report to the Secretary of Commerce by the Advisory Committee on Applications of Machines to Patent Office Operations", Ref. 46, Appendix I, p. 32; Appendix III, p. 56.

3/ See Section 3.2.1. of this report. 
one end with 207 small holes. Each hole represents a geologic subject, a geologic time, date of writing, or geographic area within the Rocky Mountains.

"Every article included in the file has been 'coded' by a geologist to indicate area, subject, and geologic time covered by the article. The geologist's notations appear on the card in microfilm form and also as slots extended from the holes in the punched end of the card. These slots make possible the selection of material by subjects. A needlesorting system is used... In selecting cards by subjects, one may use as many qualifying coding categories as desired..." 1 /

Another specific use of the Micro-Research System has been proposed for the field of neurology.

4. FACSI (Fast Access, Coded Small Images) Inc., of

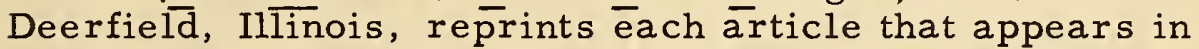
the Journal of the Society for Nondestructive Testing. This is accomplished by printing each article onto an $8^{\prime \prime} \times 101 / 2$ " edge-punched card at about a 3 to 1 reduction. These cards, purchased in sets, are indexed and prepunched with a code specifically designed for the nondestructive testing literature. The article-cards are then stored in any order and are retrieved from file by needle-sort selection on the basis of search parameters that consist of any number of applicable subject/function keywords. The retrieval process takes only a few moments to yield an article(s) that fills the search requirements. The required information is then readable with at least a loy-power magnifying glass, if not with the naked eye. $\underline{2}$

By 1956 and in fact from 1945 onward, several factors have emerged which demanded and hence fostered an increasing interest in the development of practical information selection systems that retrieve replica copies. First, there have been many important advances in data processing techniques and in the fields of mechanics, optics, electronics, and photography. Secondly, there has been a significant increase in the serious attention given to the

1/ Chronic, J. "How microfilm library aids research", Ref. 53. See also Ref. 215 , p. 16.

2/ Staats, H.N. "Data extraction in nondestructive testing", Ref. 265. 
field of documentation, especially the development of new and nonconventional systems 1 / for storage, selection, and retrieval. In part, new technological possibilities have sparked a fresh approach to the problems of classifying, categorizing, indexing, and encoding information so as to expedite future retrospective search. On the other hand, new or newly revived documentation techniques, such as the development of descriptors, uniterms, and the principles of coordinate indexing, $2 /$ lend themselves effectively to machine implementation.

A third and most important factor has been that of increasing recognition of need. The exponential growth of scientific literature has forced recognition that this problem is one of the most critical of our time and that the best utilization of available intellectual and technical resources must be applied to it. The need for better use of scientific information thus demands the continuing development of new selection and retrieval systems. In turn, serious concern for the possibilities of new systems requires not only the exploitation of current technology but the development of new techniques as well.

For this reason, the report of the Bush Committee, established to advise on the application of machines to Patent Office search, includes a review of the state of the art of data handling. This was prepared as of December 1954. It covered various advantages and disadvantages of the punched card approach, the magnetic tape approach, the photographic approach with respect to rolls and chips, and the use of high-speed electronic data processing techniques. Devices which meet the compressed storage, selection, and replica retrieval definitions of the present report were discussed. These included the Goldberg patent, the Rapid Selector, the Filmorex System, Minicards, the Recall Film Index System, and Filmsort Aperture Cards. Other aspects of the then state of the art with respect to systems providing replica copies were described in part as follows:

"An outstanding advantage of the photographic technique is that graphic material can be directly recorded and is thereafter

1/

For example, as early as 1947, Mooers suggested an application of a Rapid Selector type device that would incorporate Boolean manipulations for selection. See Ref. 188.

2/ A note by Watson Davis, recording a conversation on November 15, 1932, stated that Dr. Bush "favored the use of large sheets of film, say $20 \times 12$ inches or more, and a coordinate method of indexing. "See Ref. 65. While this terminology may have been intended to refer only to the engineering sense of 'indexing', in view of the later Bush proposals on "Memex" (Ref. 43), it may also be of historical interest. 
available for human inspection, even if it has been greatly reduced in scale, by enlarging the image in projection or during reproduction.

"To date, the conventional use of photographic methods for compressed data storage has been in the form of microfilm. The microfilm is relatively permanent and quite durable, but it cannot be erased or directly revised, and it is a scroll-like medium that must be read through from the beginning until the desired material is reached.

"However, electronic means of scanning can be coupled with coded digital information accompanying frames of text so that this searching procedure can be carried out by machine...

"The microfilm selector equipment... has definite advantages for searches where the desired result is the facsimile reproduction of text, drawings and other graphic material, or abstracts. Its chief disadvantages are that the medium requires a serial selection, that reproduction is a separate process of development, that the area for coded selection is limited by the space required for the text material, that the coded entries cannot be changed or added to without recopying or splicing the entire roll, and that the roll imposes a fixed physical grouping, of the material which must be determined at the time of recording.

"Some of these disadvantages have been attacked by systems using microphotographic media on which both text and coded index data are recorded but where the long lengths of microfilm are divided up into discrete pieces or cards. Thus the advantages in handling, regrouping, and direct selection of punched cards are combined with the advantages of data compression and reproducibility of graphic material....

"In the microfilm card approach, then, the photographic advantages of compact storage with large data capacity and facsimile reproducibility are retained, and the punched card advantages in the treatment of each record as a discrete unit in sorting, selection, removing from and adding to the files, and re-arrangement of selected groups of records, are added. The disadvantages include the limitations of photographic processing, the non-erasability of the medium, and the fact that separate conversion processes are now necessary to make information stored in this form available to other types of processing equipment...

"The photographic approach is particularly attractive where it is desired to obtain facsimile copies of graphic as well as text material... The use of photographic media is thus favorable for large collections of permanent material 
where the information stored changes slowly or not at all but where rapid access to both specific records and large groups of records is necessary. It provides compact, inexpensive, and effective storage in situations where copies of the original material are frequently required." 1 )

Possibilities for improvements and for combination techniques, such as the use of magnetic recording for selection codes for microfilmed information, were also considered by the Bush Committee. Some of these, as well as other developments not anticipated at that time, have occurred since submission of the Bush Committee report in late 1955. In 1956 Magnavox announced its Film-Data Recorder and Reader. In 1957 Benson-Lehner designed and built the system known as the FLIP, or Film Library Instantaneous Presentation. Both of these machines search a limited number of bits (which are in essence an address), then stop and project the appropriate frame. Experimental systems using video magnetic tape or a magnetic aperture card have been partially tested by RCA, Ampex, and Magnavox. In addition to the rolled film and film chip systems, several devices using micro-images on large sheets, planes, or scrolls have been built. Of these, the NBS Microcite is apparently the only operational one that locates the desired images by subject content codes at the present time.

One group of information retrieval devices has been excluded from this survey because of the special-purpose nature of its application. This group includes two types of data display systems. The first of these has the capability for retrieving a limited number of items such as maps or charts for immediate visual display. In general, the selection of the items to be displayed is by specification of the file address, and the selector mechanism is just a part of a teaching machine or a small part of a large computer-controlled system. The other type is similar, but it regenerates for display specific information from a small file, the contents of which are subject to frequent changes, often on a real-time basis, for example, as seen in air traffic control systems.

Selection and retrieval devices designed for the more usual purpose of handling scientific information are listed in Table I. Many of these devices have the same advantages and disadvantages as were considered by the Bush Committee in 1954. Consideration of the comparative characteristics of these devices however provides the framework for a current appraisal of the state of the art in systems providing replica copies. We shall therefore consider each

1/ "Report to the Secretary of Commerce by the Advisory Committee on Application of Machines to Patent Office Operations", Ref. 46, Appendix III, pp. 54-57. 
of the devices listed in Table I, but with major emphasis on those particular devices which are employed in search-type, rather than address-type, selection systems.

Table I. Document Retrieval Devices

$\mathrm{H}$ refers to known operating hardware; $M$ indicates model;

C means proved components but no complete model;

$P$ is a paper proposal.

Search type selection:

Continuous rolled film systems:

H 1938 Rapid Selector,

Bush, Green--ERA--Shaw, Yale, NBS, BuShips

H 1956 FOSDIC II, NBS--Weather Bureau

H 1961 FileSearch machine, FMA

M 1961 Automatic Image Retrieval System, Recordak

C 1960 Video File System, RCA

C 1961 70-mm Selector, Photo Devices, Inc.

C 1961 ECCETRON, Loquin

P 1959 Photo-magnetic system, James (IBM)

Chip or discrete unit systems:

H 1956 Micro Research Card, Petroleum Research Co.

H 1956 Filmorex

H 1958 Minicard, Eastman Kodak

C 1960 Magnavue, Magnavox

P 1959 Data Bank ("Is there a Doctor in the House?"), Benson-Lehner

Sheet or strip film systems:

H 1960 Microcite, NBS

M 1960 Document Storage and Retrieval System, Staats

C 1961 Large Capacity Document Storage and Retrieval System, (Walnut), IBM

Address type selection:

Continuous rolled film systems:

H 1956 Film Data-Re corder and Reader, Magnavox

H 1957 FLIP, Benson-Lehner

H 1960 Lodestar with Kodamatic or Counter, Recordak

H 1960 AutoTutor Western Design--USI

H 1961 Rapid Access Look-up System, Ferranti

H 1961 Rapid Random Access Projector, Mast Dev. Co.

M 1961 Viewer reproducer Model C and E, Hughes

P 1961 Reader-Finder, MIT 
Table I. Document Retrieval Devices (Continued)

Chip or discrete unit systems:

M 1960 MEDIA, Magnavox

M 1960 Telecard (aperture), General Precision

M 1961 Aperture card, Hughes Model D

M 1962 Target Map Coordinate Locator, Fairchild

C 1961 Automatic Unit Research Storage and Retrieval

Device, Houston Fearless

P 1961 Metricard, Thompson Ramo-Wooldridge

Sheet or strip film systems:

H 1955 Micro-image locator, NBS

H 1960 Verac 903, Avco

M 1957 Amfis, Avakian

P 1961 Information Retrieval Corp., Information for Industry, Inc. (Amfis) CRIS 


\section{COMPARATIVE CHARACTERISTICS OF AVAILABLE AND PROPOSED SYSTEMS}

As shown in Table I, the various available and proposed systems may be grouped in terms of whether the storage medium used is of continuous rolled film, chip, or discrete unit record, or sheet or strip film. Within each grouping by storage method, the se devices may be further divided by whether selection is made on a searchprescription or on an address-locating basis. The specific characteristics of those devices which use search type selection will be discussed in detail below, following the order of Table I. In addition, selected examples of microfilm aperture card systems and other address-type systems will be briefly described.

In the systems considered in this report, the storage media used consist either of printed matter on paper or card stock, or of photographic images 17 on triacetate or polyester base, or of magnetic tape recordings on polyester base. There are other possibilities for use as storage media, such as the thermoplastic recording of the General Electric Company, $2 /$ the photochromic techniques of the National Cash Register Company, $3 /$ and the fluorescentrecording materiels being studied at the University of Chicago. 1 /However, to date these storage materials are not yet being used in document retrieval systems.

Certain general characteristics of storage media therefore affect the systems to be discussed. The materials now used for storage of documents and other information items in these systems all have good life. They are stable at temperatures of $60^{\circ} \mathrm{F}$ to $80^{\circ} \mathrm{F}$ and relative humidities of $40 \%$ to $60 \%$. Ideal storage conditions are darkness, temperature of $500 \mathrm{~F}$, and relative humidity of $50 \%$.

However, if necessary, they can be used throughout an ambient temperature range equal to or greater than that acceptable to human operators, i.e., $0^{\circ}$ to $140^{\circ} \mathrm{F}$. Since all media will support the growth of microorganisms under hot and humid conditions unless especially treated they must be adequately protected.

1/ The most common emulsions used are the silver halides and the diazo dyes and light-scattering materials, see Refs. 22, 51, 80, 125,240 and 247 .

2/ See Refs., 103 and 104 .

3/ Ref. 48 .

4/ Ref. 155. 
The most serious conditions which may be encountered in the working environment are wide variations in humidity, dirt, and dust. These will adversely affect the life and use of these storage materials. Paper stock and gelatin emulsion are very sensitive to moisture content, both high and low. Materials bearing photographic images and magnetic tape media are very sensitive to dirt and dust. The adverse effects may include false readings of the information in the selection code area; for example, by the reading of a speck of dirt as a recorded bit, by failure to read a mark that has been partially or wholly obliterated, or by dirt on the tape or film that holds it too far away from the reading device. Obliteration or false reading of both code and the information items proper may result from abrasive action which scratches or wears the recording medium and base. Also, the effect on film life has not as yet been determined for sudden stopping and starting of roll film in a high-speed transport.

Characteristics which differ from one device to another include the kind or type of basic storage media, physical characteristics such as packing density, storage space requirements, the kind of input equipment required, the format of the selection code, the search and selection mechanisms, the search method, the form of output, and the like. The characteristics which were specifically considered in evaluating systems for this report are presented in Table II. 
I. General Characteristics

A. Name, developer, manufacturer, and current status.

B. Type of device - selection method, storage media, document size, code size and format.

C. Search speed, store size, and system expandability.

D. Approximate size and cost of equipment.

E. Power requirements.

II. Basic Storage Media

A. Type or kind.

B. Physical characteristics.

1. Image format and location tolerance.

2. Resolution and gray scale.

3. Method of handling, life, erasability, costs.

III. Input Equipment
A. Type of recording device and associated equipment.
B. Method of handling codes and documents - provision for parity, check.
C. Speed of transcription, ease of adjustment, and reduction ratio.
D. Equipment and recording costs.

IV. Search Equipment
A. Type of device and method of search.
B. Method of setting up question.
C. Form and speed of output.
D. Equipment and search costs.

V. Auxiliary Equipment
A. Type and purpose.
B. Costs. 
3. 1. 1 Rapid Selector $1 /$

Because of the direct line of descent from the Bush Microfilm Selector of 1938, it is appropriate to consider first the operational Rapid Selector as developed at the National Bureau of Standards for the Bureau of Ships, Department of the Navy. There are three of these systems presently in operation. The first is an NBS modification of a model developed at Yale University. The second is a subsequent model in use in the Publications Branch of the Bureau of Ships. The third is a later experimental model located at NBS. Still another preproduction model is now being constructed jointly by the Data Processing Systems Division of NBS and the Engineering Development Branch of the Bureau of the Census for the Bureau of Ships.

Much of the work on these machines has been of an experimental nature in that the Navy has been chiefly concerned with the study of indexing methods and encoding procedures, while NBS has been concerned with system development and flexibility. The Bureau of Ships Rapid Selector is used as part of a complete system for document generation, ranging from a sequenced card-camera system of text composition 2 to a facsimile-distribution system. $3 /$ The Selector System at NBS has been used mostly for engineering investigations such as film life studies and improving methods of logic control. Also, a variable reduction ratio camera and a new film transport are currently under construction.

Rapid Selector characteristics include first of all provision for selection either by a simple address or by descriptive terms and indexing entries. Combination searches can also be made in which both indexing terms and bibliographic data are used to select a desired item. In addition, there is a "predictive search" mode of operation in which counters record the number of items that meet the

1/ Refs. 13, 14, 17, 85, 110, 128, 129, 130, 131, 173, 177, 209, $234,257,258,261,262,263,285,305,307,308$ and 309 .

2/ Ball, H.R. "A photographic system for data retrieval and presentation", Ref. 30 .

Ball, H. R. "Information retrieval in the Bureau of Ships", Refs. 18 and 19.

3/ Ball, H.R. "Bureau of Ships high speed facsimile printout", Ref. 16. 
search requirements, so that the querist may broaden or narrow the scope of his search before actual copies of selected items are made.

The basic storage medium for the Rapid Selector is standard 35-mm unperforated microfilm. The document recording area is nominally 1. $15^{\prime \prime} \times 1.75^{\prime \prime}$, but the latter dimension may be extended to any desired length. This area is to accommodate documents that range in size from $3^{\prime \prime} \times 5^{\prime \prime}$ to $22^{\prime \prime} \times 34^{\prime \prime}$, covering abstract cards to engineering drawings. The selection code area is $1.20^{\prime \prime} \times 0.36^{\prime \prime}$ per 315-bit field. This provides 280 bits for document coding, which are divided into 70 hexadecimal characters, i.e., four bits per character. Any number of such fields are available for encoding.

The microfilm can be easily handled on 100 foot to 6,000 foot reels, representing 600 to 36,000 coded pages per reel. As many reels as desired may be used in the total system. Other packing density characteristics available in the system are (1) a resolution capability of up to 120 lines per $\mathrm{mm}$, and (2) six coded frames per foot. 2 The storage space requirement for a 6,000 foot reel is $2^{\prime \prime} \times 20.5^{\prime \prime}$, or 660 cubic inches. Under normal conditions film storage life is indefinite. However, for its handling, a controlled atmosphere is desirable.

The experimental input equipment for the Rapid Selectors is shown in Figure 4. It is a modified commercial microfilm camera for photographic recording of documents and other information items at a reduction ratio of 8 to 1 and a resolution of 60 lines per $\mathrm{mm}$ across the entire field. For recording of the selection criteria information, a modified IBM 407 card reader is used. This reader has been altered to read all hole positions simultaneously. Each hole position sensor is connected to an independent light source in the code matrix as seen under the table in Figure 4. These lights are photographed onto the film as the code pattern. To prepare the cards for recording, an IBM 026 card punch has been modified to punch hexadecimal characters in each column of the encoding cards. In addition to punching the characters in a specified 4 rows, it also punches the complement of the characters in 4 other rows of the same columns. This feature also makes the punch useful for

1/ McMurray, J.P. "The Bureau of Ships Rapid Selector System", Ref. 177 , p. 4.

2/ This may be increased to 36 frames per foot where a punched card is recorded as both document and code storage area, e.g., such as the case in which titles only are used. 


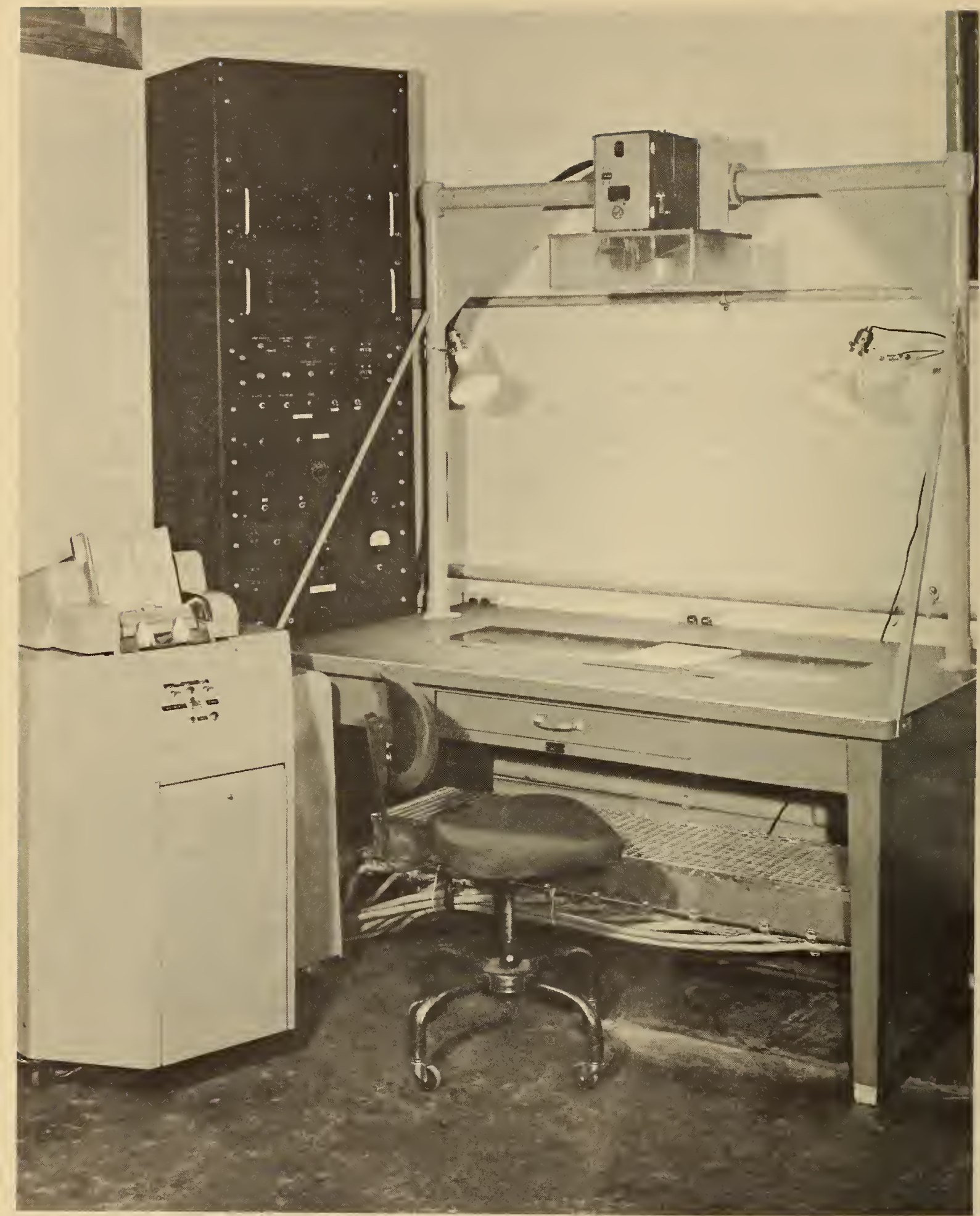

Figure 4. Rapid Selector Encoder 
preparing the question cards to be used for interrogating the Selector because the program logic requires the complement of the characters as well as the characters themselves.

The input operation thus consists of punching the selection code information into a card with a manual keyboard. Cards are fed semiautomatically from a hopper. The lights corresponding to the holes punched in the card come on, and a picture of the code pattern is taken by the camera. For each document, this is repeated for as many cards as are required for the document code. A switch is then thrown to turn on flood lights and refocus the camera onto the table top where the documents are placed. As many document pages can be photographed as are required. The original film is edited, spliced, and retained as a security file. From that copy, as many machine master copies are made as are needed.

The search equipment for the Rapid Selector consists basically of a high-speed mechanical film transport, a photocell-activated interrogator and comparator, and an on-the-fly output copy camera. Search prescription requirements are established by punching the codes with the desired selection criteria into a question card. "Yes", "no", and "don't care" conditions are available through use of the character complements mentioned above. This card, together with a patch panel, permits logical relationships between the prescribed criteria. Together they set the comparator-identification circuitry.

The search method is serial, i.e., item by item as recorded on the master film. The selection code area for each item is read 40 bits at a time for the purpose of matching the photocell comparatoridentification circuit to the search prescription. As previously noted, the present code field capacity is in groups of 280 bits. Each individual bit in the field may be checked for "yes, " "no, " and "don't care" conditions. Bits may also be combined logically within a group.

In operation, the master film is run continuously at a uniform velocity. The paths of the master and recopy films are shown schematically in Figure 5 and may also be traced in the photograph of the Rapid Selector search equipment (Figure 6). On this particular model both films are driven by a single drive drum. Whenever a searchpattern match identification is obtained on the master film, the copy film is brought up to proper speed by the clutch and a copy is made. A delay mechanism is required for the clutch control signals since the selected area on the master film does not reach the copy station until some time after it passes the code recognition station. The clutches used in two of the operational models will accelerate the copy film to the full velocity of $5^{\prime}$ per second in 3 milliseconds. The amount of film passing through the copy gate during this period is about equal to that required to get unexposed copy film to copying position. The distance between the selection gate and the copy gate is enough so that the acceleration time is of negligible concern. Good alignment of 
MASTER FILM TAKE UP REEL

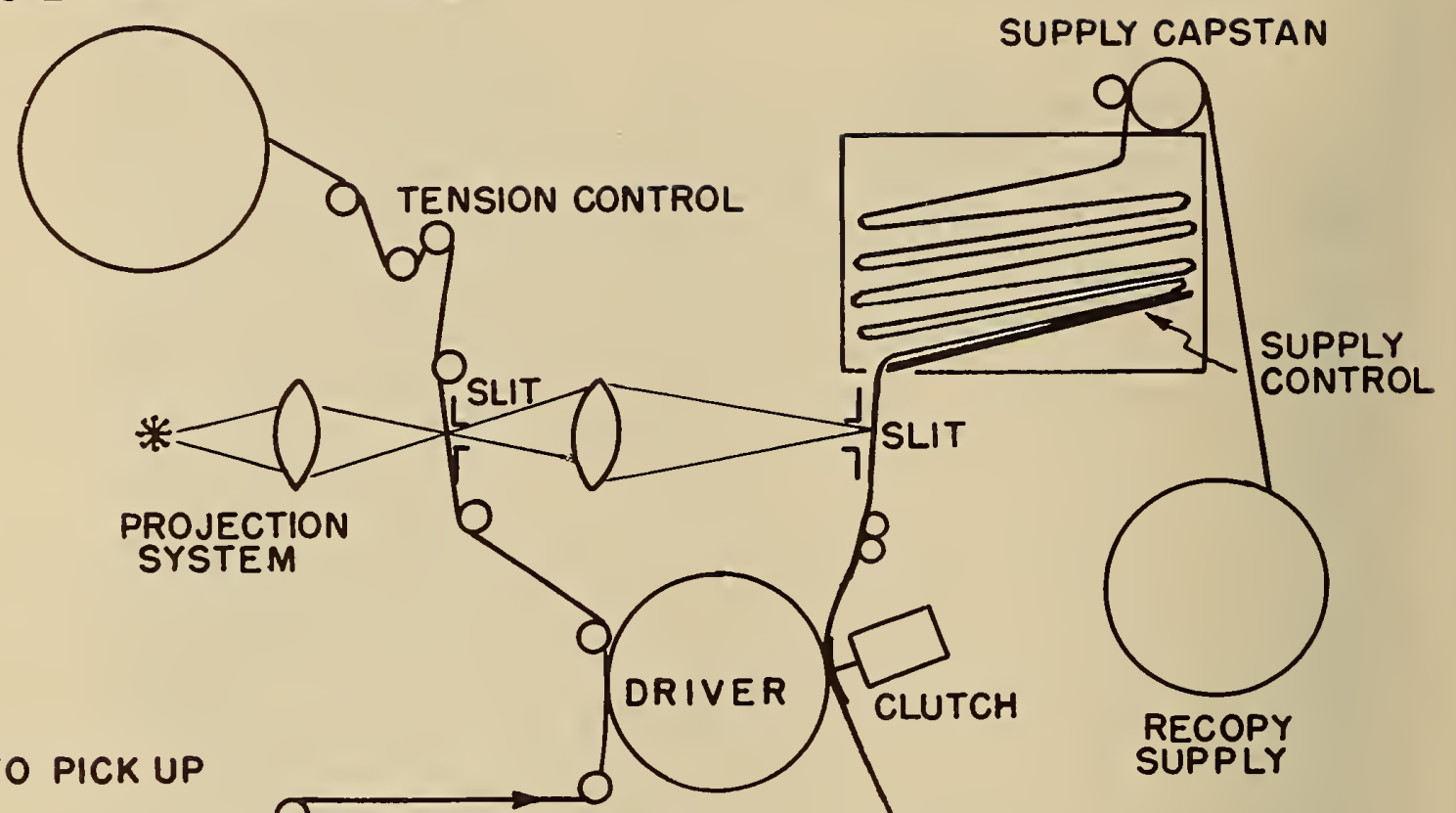

PHOTO PICK UP
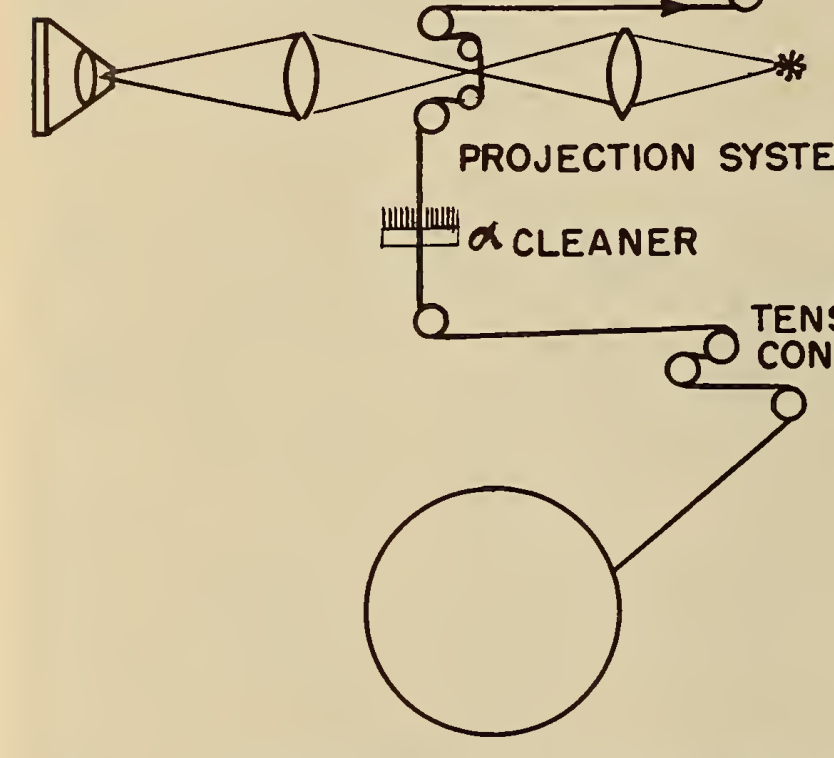

MASTER FILM SUPPLY REEL

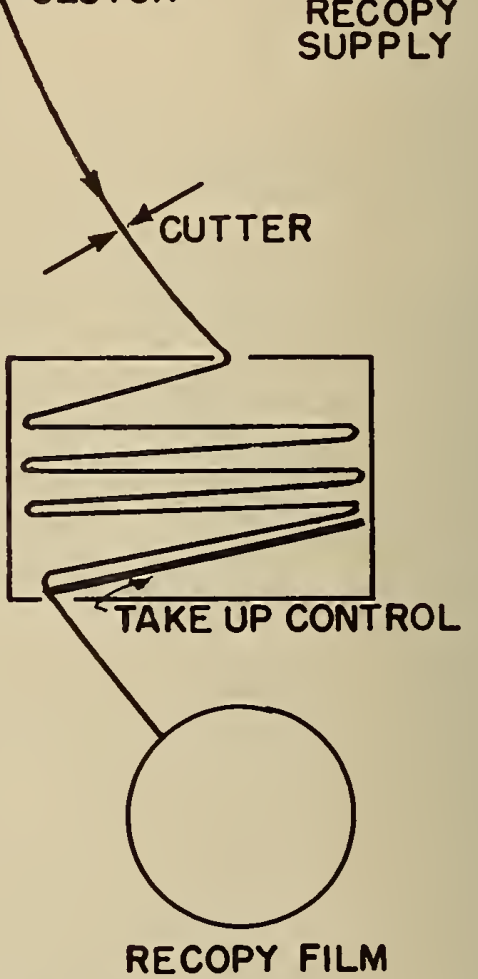

Figure 5. Rapid Selector Film Transport Schematic 


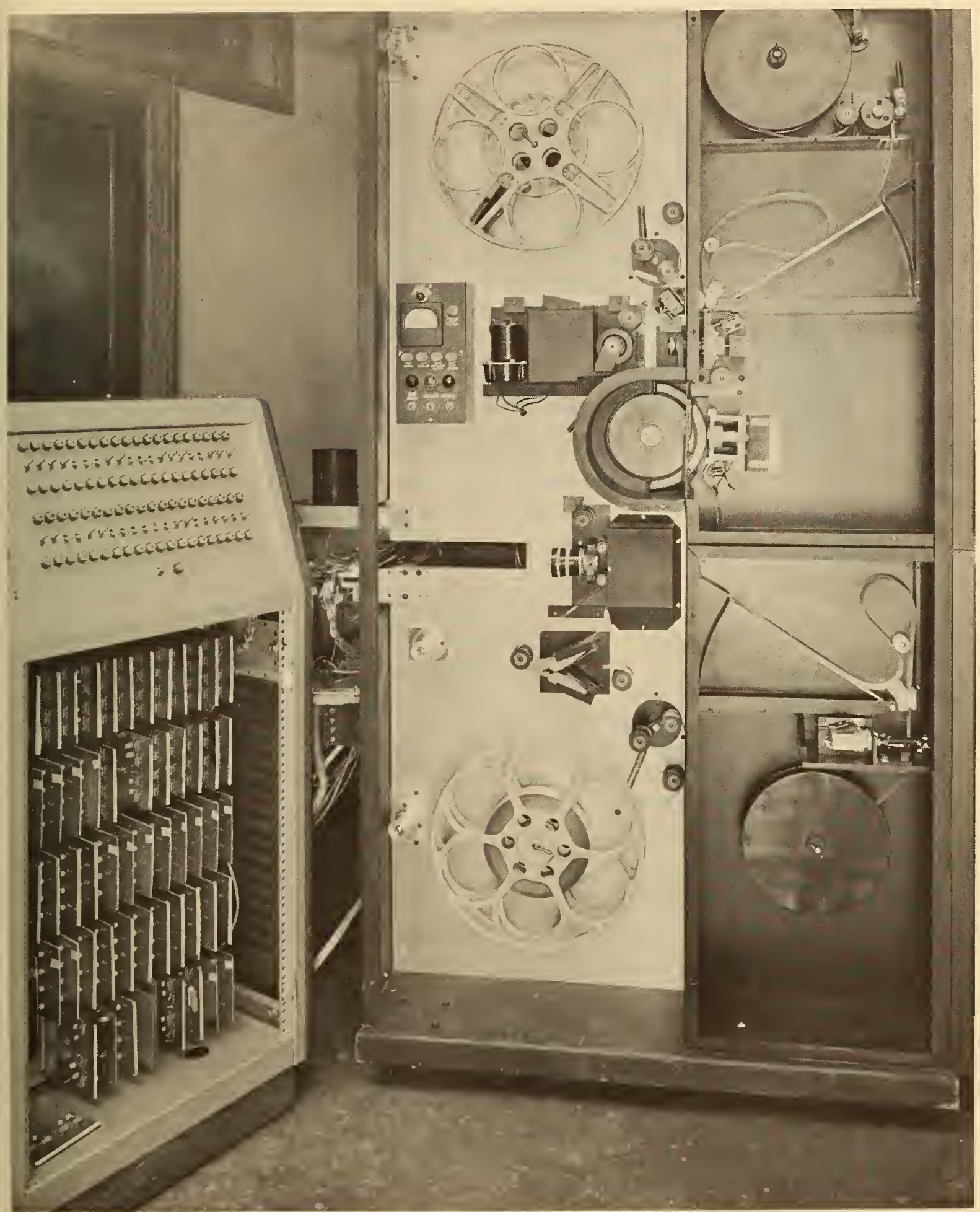

Figure 6. Rapid Selector 
the film in the selection gate is necessary for proper code recognition. Also, the films must be flat in the copying gates to give clear undistorted copy. In addition, there are interlocks in the film paths which automatically shut the machine down if either the master film or the copy film breaks, or if the copy film runs out.

The output of the Rapid Selector is a 1 -to-1 copy of the $35-\mathrm{mm}$ microfilm image of the original document or information item. In principle, the file items can range from titles, through abstracts, to full text. In the case of the Bureau of Ships Rapid Selector, this has included "textual material, engineering drawings, maps and halftones". 1/ The direct output can be viewed by system customers on various commercially available microfilm viewers. Alternately, an auxiliary enlargement-printing process may be employed to provide full-size replicas. With microfilm, gray-scale or tonal quality is relatively poor. However, the Rapid Selector system can be used effectively with other types of films that have good tonal range.

Performance of the Rapid Selector system, as with any document retrieval system, is of course related to the requirements of the specific application. In the case of the Bureau of Ships installation, an information specialist analyzes each document and chooses the terms to be used for identification and subsequent selection. At least one of these terms is chosen from the Navy-Marine Corps Standard: Subject Classification System Manual 2 /. This term, in effect, limits or controls the applicability of other descriptors. In addition, a screening descriptor, such as "research report" or "operating manual", is assigned to describe the type of item. Role indicators are also sometimes used to modify the application of an assigned subject descriptor.

Within the above analytical restraints, performance has been reported to involve an average time of 20 minutes per item for document analysis and indexing, with an assigned average of nine discrete subject entries and 12 bibliographic entries such as author, title, date, and journal or report.

For 1,200 retrievable items initially recorded in the Bureau of Ships system, the average number of searches per month has been 150. The average search handling time has been 12 minutes. Theoretically, the rate of search is 36 coded pages per second, or 1,960 coded pages per minute for a full reel. However, the time required to locate a particular item depends upon the length of the

\footnotetext{
1/ McMurray, J.P. "The Bureau of Ships Rapid Selector System", Ref. 177, p. 3.

2/ Ref. 286.
} 
master reel and amounts to an average of six minutes for a full 2,000-foot reel $(12,000$ coded items). 1/

There are a number of overall system features and operational and maintenance characteristics that are pertinent to system design considerations. The total cost of the input equipment is approximately $\$ 35,000.00$ on a one of a kind basis. The cost of auxiliary equipment (modified card punch, Kalvar microfilm copier, modified Fairchild self-threading processor) may amount to an additional $\$ 10,000.00$. Operator time, including manual placement of documents to be photographed, is estimated at 1 minute per complete item. A 100 foot reel of film costs $\$ 6.50$ including processing. The search equipment in the Rapid Selector system would probably cost approximately $\$ 40,000.00$ for a prototype model. In general, operational costs are estimated at $\$ 0.05$ per page for file input and $\$ 0.03$ for each page retrieved. Space required for the system includes storage space for as many reels as may be required, $3^{\prime} \times 5^{\prime} \times 8^{\prime}$ plus $3^{\prime} \times 3^{\prime} \times 4^{\prime}$ for the input equipment, and $4^{\prime} \times 2^{\prime} \times 6^{\prime}$ plus $2^{\prime} \times 2^{\prime} \times 4^{\prime}$ for the search equipment. Power requirements for the input and the search equipments are 10A, $115 \mathrm{~V}$ and $20 \mathrm{~A}, 115 \mathrm{~V}$ respectively.

The storage medium is neither erasable nor reversible. Therefore, insertion and deletion of items must be accomplished by a manual cut-and-splice process. Similar manual operations are required for both replacing damaged material and for rearranging the item order. For input, the only provision for manual insertion or code correction is that the code cara may be corrected and replaced. There is no provision for verifying and checking the coded information other than by visual inspection. However, file integrity is maintained, since the master film always remains in the file area.

Developments similar to those for the Rapid Selector are covered in several other systems discussed in this report. In addition, an apparently related development in the USSR is referred to as an "automatic catalog-handbook". In particular, this device has been reported as follows:

"On a film strip is printed on one side annotation of articles, on the other side an index to them. The index consists of black and white dots. Automatic devices with photo-electric cells scan such bibliographical cards up to $10^{\prime} \mathrm{s}$ of $1000^{\prime} \mathrm{s}$ per minute. In 6 or 7 minutes the automatic librarian is able to find any one of 70,000 references recorded on the strip. " $\underline{2}$

1/

McMurray, J. P. "The Bureau of Ships Rapid Selector System," Ref. 177, p. 1.

Private communication, Melville J. Ruggles, enclosing his translation and abstract notes on an article in a Soviet journal, by V. Pekelis, published in 1958. 
Also, a similar device is purportedly being developed by Marcel Loquin of Paris. In it, $16 \mathrm{~mm}$ film is used for storing the index code words adjacent to the documents. Search is performed by optically matching a projected image of the index word with a mask of the word. The original and masked code words are inscribed by a conventional typewriter (the same for both). When a match is made, the film is stopped and the document area projected onto a reading screen. This unit is called ECCETRON.

\section{1.2 FOSDIC II 1 /}

The second device in the continuous rolled film, search-type selection category is, interestingly enough, a retrieval system that uses the document information in its original form as the selection code. The FOSDIC II system is one of several different types of FOSDIC (Film Optical Scanning Device for Input to Computers) equipment. The FOSDIC systems have been designed at the National Bureau of Standards to meet the special needs of the Bureau of the Census and other Government agencies. FOSDIC II, developed for the U.S. Weather Bureau, represents a special case of the FOSDIC systems in which microfilm is used as the storage media, automatic selection is made of certain specific records on the basis of information contained in these records, and an exact replica is provided as output. 2 ]

The FOSDIC II system was specifically designed to meet the needs of the National Weather Records Center. At this Center, more than 450,000, 000 punched cards containing climatological information must be maintained on file, and the file grows at the rate of approximately 35,000,000 cards per year. For economy, compactness, permanence and security of storage, the original punched cards are microfilmed. FOSDIC II is used to search the microfilmed file and to select those items which meet specified requirements, e.g. , a given combination of wind velocity and barometric pressure in a certain geographical region.

The retrieval output from FOSDIC II is an exact duplicate of the original punched card. It is on card stock and is punched to carry all of the data originally recorded. A second FOSDIC II system, which will read twice as many items per minute. is being tested. This secönd model can generate magnetic tape recordings for direct input

1/ Refs. 96, 97, and 112 .

2/ That is, one in which the physical dimensions of the original, including card thickness, are at least as important as the graphic facsimile aspect. 
to computer, and thus can provide another output mode as well as the replica copy output feature.

The storage medium used in FOSDIC II is $16 \mathrm{~mm}$ unperforated microfilm. The document or item area is approximately $0.075^{\prime \prime} \times 0.307^{\prime \prime}$, recorded across the film at a density of 11 cards to the inch. The selection code area exactly coincides with the document area, since the indexing and selection information can be any of the data originally punched into the card. The reduction ratios used are 24: 1 across the film and 43.5:1 along the film; so that the original rectangular holes of an IBM card have a square image after microphotography. The handling units are 100 foot reels of film, with a capacity of 13,200 cards per reel. Reels must be hand-threaded. Cut-and-splice processes must be used to insert new items, to rearrange the order of items, or to replace damaged material. Handling, cleaning, and maintenance operations involve standard commercial processes, which for this purpose are under carefully controlled conditions to protect against dirt. Input recording is achieved by means of a modified standard microfilm camera at rates up to 800 cards per minute (Figure 7 ).

The FOSDIC II search equipment occupies five relay racks, as shown in Figure 8 , with overall dimensions of $12^{\prime} \times 6^{\prime} \times 2^{\prime}$. It combines electronic, mechanical and optical techniques. Search questions are set up by plugboard. The device can select from information recorded in any 10 columns of the 80 columns of the original card. Search is serial in terms of items, and for the 12 hole positions per card column. The search speed for the equipment now in use is at the rate of 4,000 cards per minute.

The FOSDIC II equipment thus uniquely combines integrity of the master files with the advantages both of a continuous storage medium (master cards cannot be lost or misfiled) and of the manipulation features generally available for discrete unit records. Costs of the system approximate $\$ 100,000$ for the search equipment, with standard costs for the microfilm medium, processing and duplication. The second model now under construction is designed for greater flexibility of the programmed logic, higher speed, and magnetic tape output.

\section{1.3 FileSearch $1 /$}

The File Search equipment, designed and produced by FMA, Inc. , is presently being put to operational use in the Central Records Section of the Navy Bureau of Ships. At the present time, document analysis, encoding, and machine file input is limited to a collection of Navy directives. These are being indexed for machine retrieval

$1 /$ Refs. 89, 93, 94, 99 and 269, p. 66. 


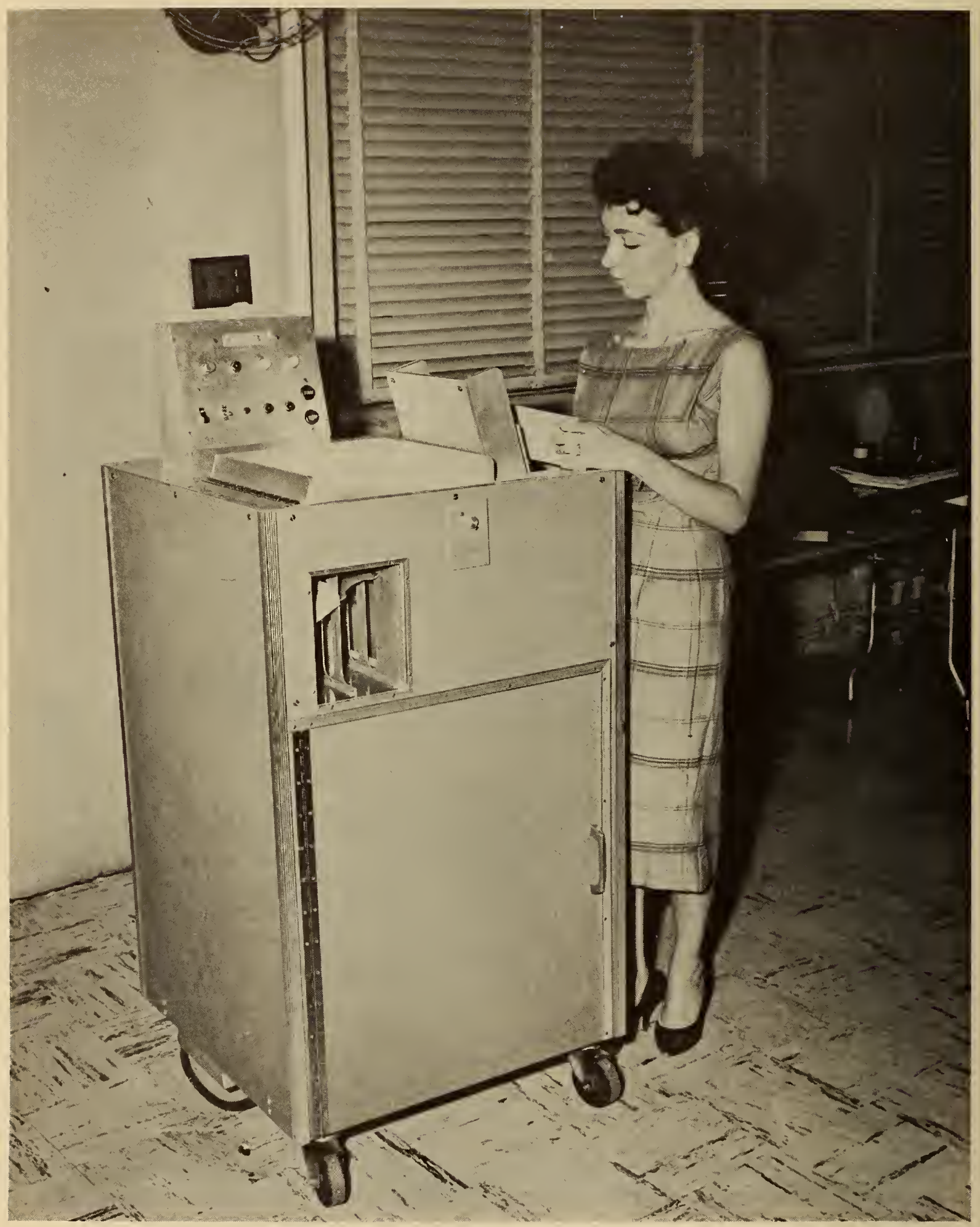

Figure 7. Fosdic II Camera 


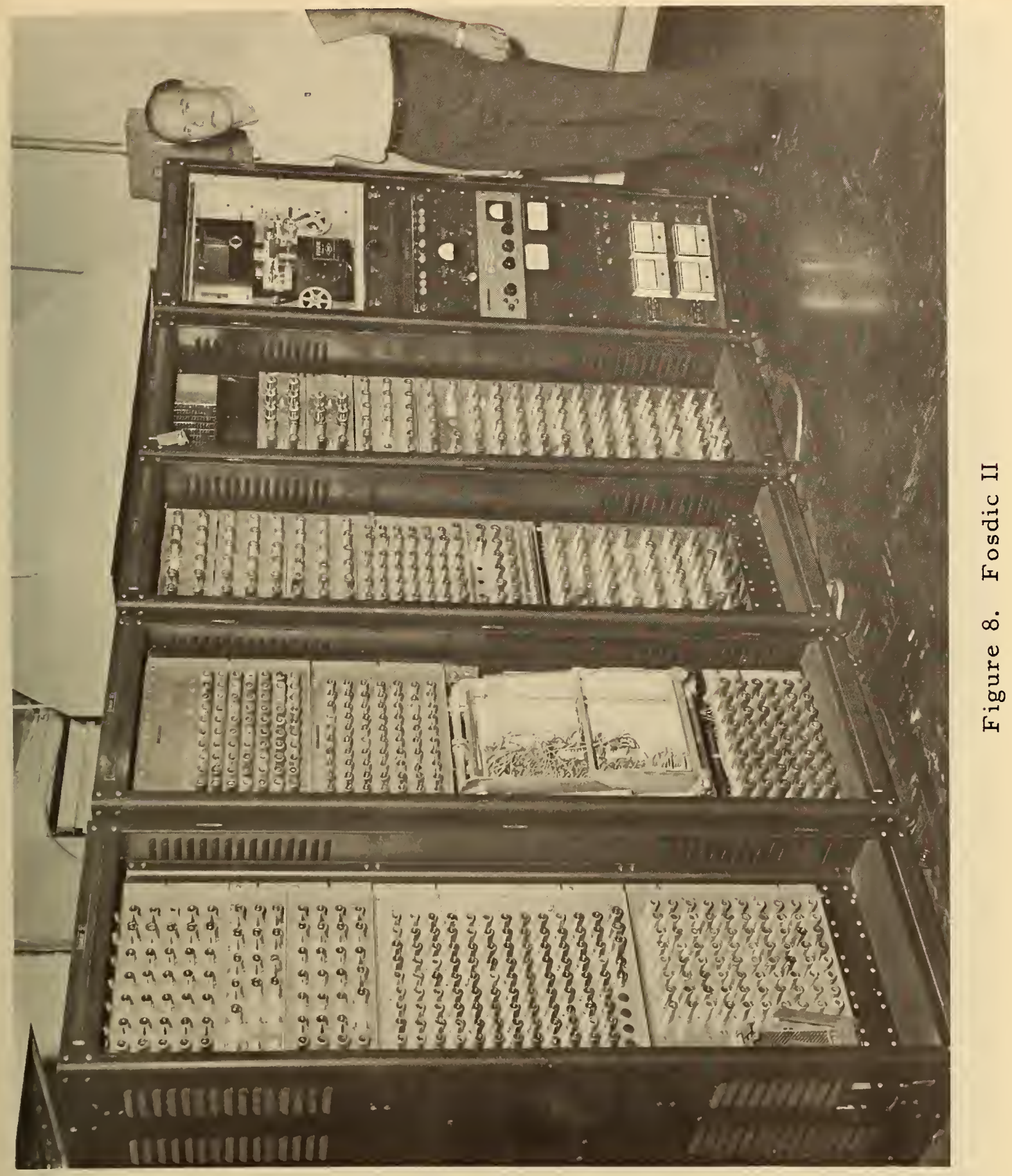


by the following selection criteria: document type, date, directive number, supplement or change number, originator, routing, and subject. The subject terms and codes used are derived from the Navy-Marine Corps Standard Subject Classification System Manual (SECNAVINST P5210.11). 1 Only one subject term is permitted per selection code card. If more than one subject term is required, a trailer card is used for the additional term. One selection code card is required for each document page. Normally, the trailer card is photographed with the second document page. Otherwise a blank document area must be left for each trailer card.

The Navy installation of FileSearch equipment consists of two Friden card punches for selection code inscription, a card cutter, two fixed-reduction cameras (both of which will take legal size document pages), and the search equipment. The camera set-up and the search equipment are shown in Figures 9 and 10, respectively. The camera requires a space of $3^{\prime} \times 4^{\prime} \times 7^{\prime}$ and the selector a space of $6^{\prime} \times 4.5^{\prime} \times 2.5^{\prime}$. Power requirements for the selector are $35 \mathrm{~A}, 115 \mathrm{~V}$.

The storage medium used in the FileSearch device is $35 \mathrm{~mm}$ perforated photographic film with a resolution of 200 lines per $\mathrm{mm}$ and a reduction ratio of $25: 1$ for page sizes up to $8.5^{\prime \prime} \times 14^{\prime \prime}$. The packing density is 32 pages per foot, or 32,000 coded pages per 1,000 -foot reel of film. The actual document area is $0.340^{\prime \prime} \times 0.560 "$ and that for the selection code is $0.280^{\prime \prime} \times 0.160^{\prime \prime}$. A maximum of 56 alphanumeric characters can be stored in this selection code area which is located beside the document page frame. Characters are in the form of 7-bit code, including a parity check bit. As in most other microfilm selector equipment, each bit is represented by a small area (0.010" $\times 0.010^{\prime \prime}$ in the FileSearch format) which can be either transparent or opaque.

The input operations require first that the selection codes be punched into Friden cards, 56 characters per card. Documents are placed by hand on a table. The card is inserted into the read slot of a special card reader having a fiber optics bundle which reorients the bits for photography into rows of 14 information bits each. When the camera is activated, it records the light array and the document simultaneously. Processing of complete items for file input proceeds at rates ranging from 180 to 400 finished frames of film per hour, depending on the experience of the operator.

For purposes of search, selection, and retrieval with this equipment, the 56 characters of the selection code area may be separated into six groups which may be used in several ways. All six groups can be logically combined to specify a single document or

Ref. 286. 


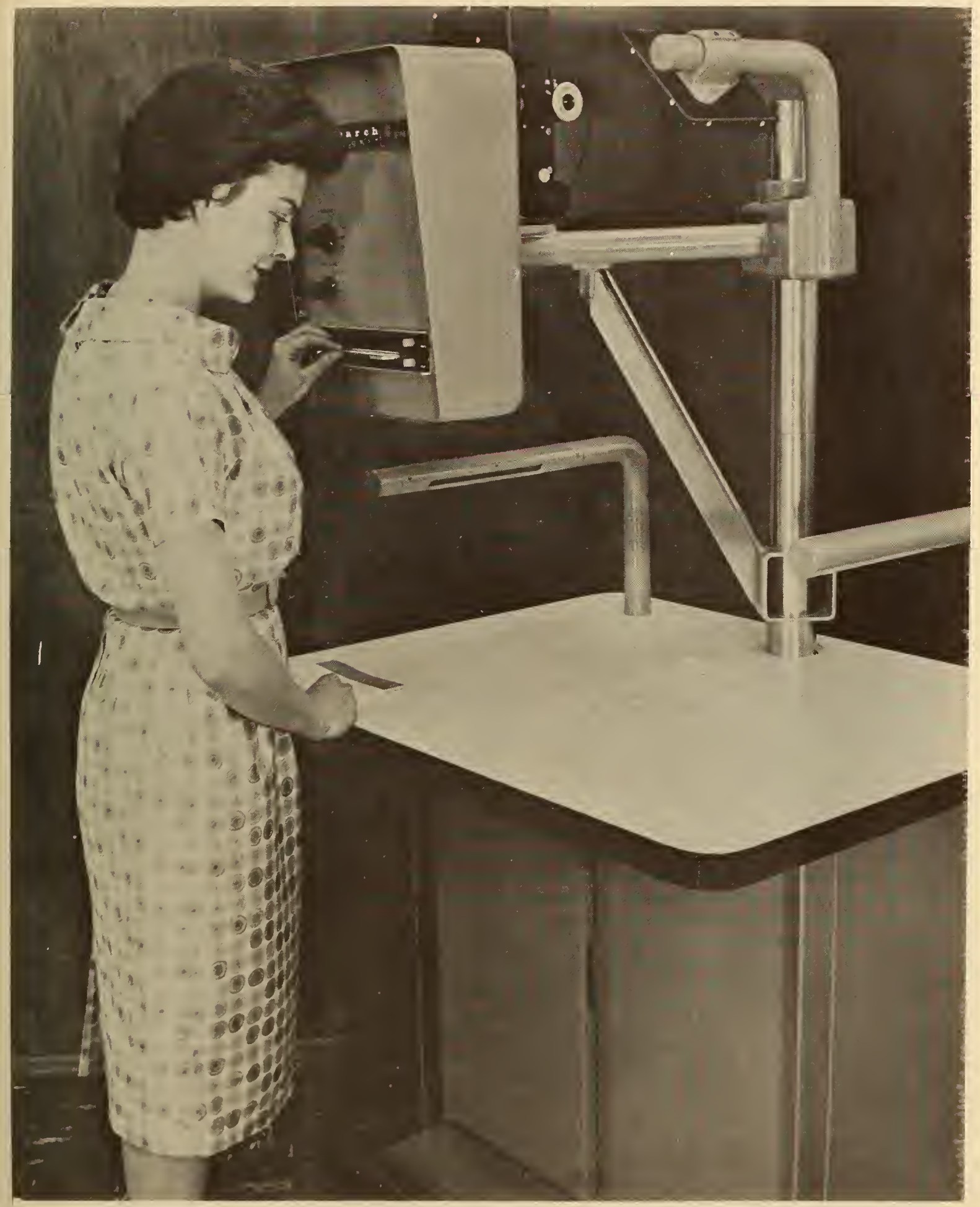

Figure 9. FileSearch Camera 


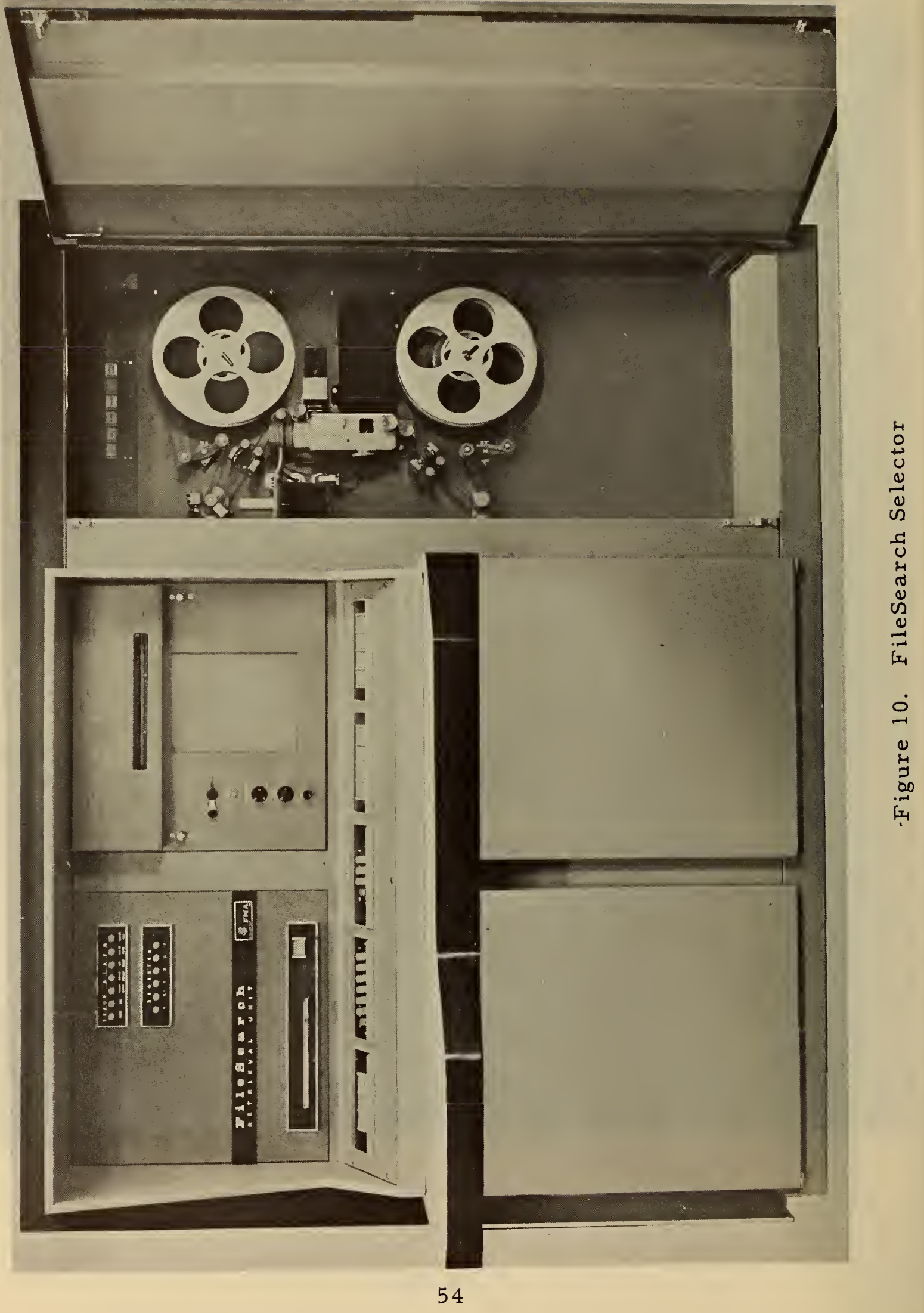


set of documents, or they may be used to describe with less specificity up to six separate sets of desired documents. In this case, the descriptor codes desired will be or-gated together, and therefore a hit will be recognized whenever any one of these codes appears for any document. Thus, a measure of multiple-search capability is provided, either for simple single-subject searches or for an addressselection mode in which the six-code groups prescribed can represent six different document numbers.

The search equipment consists essentially of a high-speed mechanical film transport, a flying spot scanner, and the recognition circuitry. When a frame is found with a code that matches the search prescription, the film is stopped and backed to viewing or copying position by means of a sprocket hole counter. The search method is serial. The logic provided includes "and", "or", "greater than", and "less than" conditions which may be combined. The output of the search can be either the projection of the image of the document for direct inspection, a 35-mm perforated microfilm copy, or a hard copy print ( $3 \mathrm{M})$, i.e., Minnesota Mining and Manufacturing Co.

The search rate can be as high as 6,400 coded items per minute provided that there are no stops, i.e., no hits. In considering search time it should be remembered that the film in the FileSearch machine is brought to a full stop for viewing or copying each time a frame is selected. "The speed of a complete search of the file is therefore a function of the number of items selected. Stopping the master film for each hit permits the searcher to make an immediate inspection of the selected item. He also can take advantage of this feature to provide a limited degree of browsing ability, since manual controls allow him to view adjacent frames as well as a selected item.

Because copying is done with the master film held stationary, greater reduction ratios can be used than is the case for systems in which copies are made dynamically, viz., on the-fly.

A controlled atmosphere is desirable for processing, handling, and cleaning. Reels are hand-threaded onto the search equipment. Cut-and-splice operations are required to replace or rearrange recorded items. The master film is never removed from the file. However, unlike the Rapid Selector, FOSDIC II, and other systems, a security film does not now, exist for this system. Therefore, loss of or damage to the master film requires the preparation of a new master film.

Costs of the equipment are approximately $\$ 15,000$ for each input camera, $\$ 88,000$ for the search unit, and $\$ 11,500$ for auxiliary equipment, such as the 1:1 file expansion camera and card cutters. 


\section{1. 4 Automatic Image Retrieval System $1 /$}

The Automatic Image Retrieval System consists of equipment under development by the Recordak Corporation, a subsidiary of Eastman Kodak. Up to the present time only the cameras for this system are being used outside the company laboratories. Three cameras are now in operation at the Defense Logistics Service Center and four selection units are scheduled to be delivered to that agency in May, 1962.

The first model uses $16 \mathrm{~mm}$ unperforated dyeback microfilm with a selection code area at present effectively limited to 24 bits or six 4-bit characters. The microfilm is packaged in 100-foot selfthreading magazines. On this film, the documents are recorded by a modified Recordak MRD2a camera in an area $0.510^{\prime \prime} \times 1.8$ ", at a reduction of 23 to 1 , and with a resolution capability of 120 lines per $\mathrm{mm}$. There are 2,000 coded pages per magazine at a packing density of 25 coded pages per foot. As for the systems previously discussed, the total system storage capacity is as many magazines as are needed for a specific collection. Each magazine has dimensions of $4.5^{\prime \prime} \times 4.5^{\prime \prime} \times 1.2^{\prime \prime}$. Thirty such magazines $(60,000$ coded pages) can be filed in a tray measuring $4.5^{\prime \prime} \times 4.5^{\prime \prime} \times 36.0^{\prime \prime}$. The Defense Logistics Services Center has a modified Diebold, Inc. tub file which contains approximately 1, 600 magazines.

Stored items are to be searched serially within each magazine. The magazines are inserted in a holder so that film is automatically threaded for search. Cutting and splicing will be necessary for rearrangement, insertions, and deletions. However, since each item in the current file is searched by a number, all replacement items bearing the same number as the original are added at the leading end of the film. While making a search then, the most recent acquisitions are found first. Searching further down the film is done only if data on a previous item bearing the same number are required.

The input operations involve the punching of the selection code entries into cards which are hand-fed into the reader while the corresponding document is manually positioned on a photographic copying table. The codes are punched in standard IBM cards. The reader modifies the Hollerith code format to binary-coded-decimal (BCD) for recording. The resultant code-light-raster and the document itself are photographed simultaneously.

The search equipment is a high-speed mechanical film transport based on the Lodestar Reader in which the master film is stopped for viewing or copying. The search requirements are set on a

1/ "Recordak Automatic Image Retrieval System",Ref. 239, and Senate Document 113, Ref. 284, p. 256. 
six-column keyboard for the desired code pattern. The initial film speed is 10 feet per second. When a hit is encountered, the film is stopped and is reversed to correct for over-shoot. A maximum of two seconds may elapse from time of match-recognition to exact location of the item. Upon the incidence of a selection-hit, the film is stopped for viewing or copying. Theoretically, the search speed would approach 250 coded pages per second or 2, 500 items per 100-foot reel in 10 seconds provided there were no hits or stops. The output is projected for inspection or printed as hard copy.

Operational requirements are similar to those noted for other systems, particularly in that a controlled atmosphere is desired. Other features noted are that broken film can be spotted by visual inspection and that initial record-processing, handling, cleaning, and duplication costs are commensurate with standard commercial microfilm practice (processing at $\$ 4.18 / 100$ feet, duplicating at $\$ 3.90 / 100$ feet, and cleaning at $\$ 2.50 / 1,000$ feet). Costs are $\$ 24,000$ for the input camera and $\$ 18,000$ for the selector equipment.

\section{1.5 Photo-magnetic System}

This proposed system was suggested by Peter James in a paper which appeared in the October, 1959 is sue of American Documentation and also appeared as an IBM research report dated March 1, 1958. If A dual storage recording system is proposed in which selection code information in the form of binary-encoded data on magnetic tape would be run simultaneously with microfilm containing the compressed images of documents to which the encoded entries refer.

The purpose of the proposed Photo-magnetic system is to provide selection and retrieval operations similar to those of the original Microfilm Rapid Selector except that the selection code identifiers would be recorded magnetically rather than photographically. Such a change from optical to magnetic recording would provide advantages of erasability, reversibility, up-dating, and revision for the selection criteria not readily available with photographic storage of the selection code entries. However, precisely these possibilities had been considered by the Bush Committee, as reported in 1954, as follows:

"Developmental work is also in progress on ways and means to combine varied data storage media for special purposes. Combinations of punched cards and microfilm are commercially available. Combinations of magnetic tape strips and punched cards are being explored.

"In the Yale version of the Rapid Selector, a magnetic recording track stripe on the film has been suggested to carry

Ref. 142 . 
pulses produced by recognition signals from the Interrogator Unit in order to activate the recopy camera. These pulses would be retained on the film after the interrogation run. This technique would make possible the transfer of the film to a separate printer so that rearrangements of selected frames could be made up into separate reels as desired. It is certainly possible that the portion of film now reserved for optically sensed recording of coded index entries could be replaced by an area for magnetic recording of similar, selection information that could be more readily revised." 1 /

The James system would provide for the combination of microfilm with magnetic tape recording for document and selection code areas, respectively. Resolutions of 120 lines per $\mathrm{mm}$ and 400 bits per inch should be easily achieved. Either a fixed or variable code field format could be used. Keyboard input might be utilized both for initial selection code inscription and for subsequent presentation of the search prescription requirements. Search would be serial and the output might be either a 1:1 replica or an enlarged hard copy print. In terms of maintenance and operating conditions, these would be similar to those encountered in the purely photographic retrieval devices. However, while document images in storage would require cutting, splicing, or recopying for new additions or arrangements, the selection code areas could be readily rewritten and revised as required.

\section{1 .6 Video File $2 /$}

The Video File system exists to date as tested components that have been built by RCA and, as such, has been suggested for several different applications. For each specific proposed application there have been variations in the number of information reading stations suggested, this number being a function of file size, speed of output desired, and estimated costs.

In one such proposed application where speed was not of major importance, a single video tape reading head station was envisaged which would handle 10 reels of 60,000 coded pages each. Under these circumstances, the average time to provide hard copy printout

1/ "Report to the Secretary of Commerce by the Advisory Committee on Application of Machines to Patent Office Operations", Ref. 46, pp. 61-62. In addition, the Morse patent previously cited (Ref. 190) also suggested magnetic recording.

2/ RCA Brochures, Ref. 231 and 232 . 
of a selected item was estimated to be six minutes. About half of this time would be necessary in order to locate the proper reel on which the selected item would be found. Although various components of the Video File system have been constructed, no complete system has as yet been assembled.

The storage medium in the RCA proposal would be high-quality 2 -inch video magnetic tape, of the general type used for television recording and playback. The document page area would occupy a space of $1.70^{\prime \prime} \times 2.25^{\prime \prime}$, with a $0.15^{\prime \prime}$ gap between each recorded page. The selection code area would provide a $0.070^{\prime \prime} \times 2.25^{\prime \prime}$ space for the information-carrying codes together with index timing and control track signals. At a resolution equal to five lines per $\mathrm{mm}$ and a reduction ratio of 5:1, six pages could be recorded per foot. The basic handling units would be 10,000-foot reels containing 60,000 pages each.

With respect to storage life, it should be noted that while the search process itself would cause little or no deterioration, the action of the read-out head would wear the information tracks to the point of uselessness within approximately 100 readings of a given area. However, the location of lost or seriously deteriorated information could usually be detected both by checks on parity bits incorporated in the codes and by visual inspection of the quality of the output.

Input operations with respect to documents to be filed would be carried out by means of a flying spot scanner which would generate video signals for recording on magnetic tape. Input of selection code inscriptions could be by manual keyboard or by various card or tape input devices. Search prescription requirements could also be set up by manual keyboard or by card or by tape input. The search process is designed to be parallel with respect to direction to the proper tape unit, but serial with respect to items recorded along each reel. Search speed per reel is estimated at 300 inches per second.

The proposed output of the Video File system would be either a projected image for human viewing or hard copy produced by Electrofax printing. A 16-channel high-speed conventional tape buffer unit would store the selected information for reproduction by the printer. Because of the buffering, multiple copies of the same selected information item can be made on programmable command.

The physical space requirements for this equipment would be $4^{\prime} \times 4^{\prime} \times 3^{\prime}$ for the input scanner, $3^{\prime} \times 6^{\prime} \times 2^{\prime}$ for the various tape units, $6^{\prime} \times 6^{\prime} \times 2^{\prime}$ for the control unit, $3^{\prime} \times 6^{\prime} \times 2^{\prime}$ for the tape buffer, $3^{\prime} \times 5^{\prime} \times 3^{\prime}$ for the viewer, and $3^{\prime} \times 5^{\prime} \times 5^{\prime}$ for the Electrofax printer. Costs are not generally available and would obviously vary with the number of component units desired in a particular installation. For several of the proposed applications, the Video File system is apparently 
intended primarily as an address-type selection and retrieval system. In principle, however, with at least 400 bits available for selection criteria coding, there is no reason why the system cannot also be used for search and selection on the basis of other descriptive and subject indexing terms.

\section{1. $770 \mathrm{~mm}$ Selector}

A prototype model for a $70 \mathrm{~mm}$ Selector-type system is currently under construction for the U.S. Naval Photographic Interpretation Center by Photo Devices, Inc. The storage medium is continuousroll $70 \mathrm{~mm}$ unperforated microfilm. The document area is $2-3 / 8^{\prime \prime} \times 21 / 2^{\prime \prime}$ and can accommodate document sizes ranging from $9^{\prime \prime} \times 9^{\prime \prime}$ to $31^{\prime \prime} \times 31^{\prime \prime}$ at reduction ratios varying between 4 to 1 and 14 to 1 . The commercially available Photo Devices Model $1088 \mathrm{~B}$ camera is being modified for this purpose. The code information will be derived from standard punched cards using the Hollerith code. The code area will therefore contain space for 960 bits in a $1^{\prime \prime} \times 21 / 4^{\prime \prime}$ area. For this model, a Richardson static card reader will be used to activate a light raster which will produce an optical image of the punched hole pattern. The raster is to be an integral part of the camera and will have its own optical system so that the variable reduction features of the camera can be used for recording the documents.

The search equipment will use a. standard punched card for control. Some logical manipulation of the codes will be provided. The search will be serial on 1000-foot reels at approximately 100 feet per minute. The output will be a $6 \mathrm{X}$ enlarged view of the search film or a $3 \mathrm{M}$ hard copy. The search electronics was built by the Computer Control Company, Inc. The input equipment will be about $5^{\prime} \times 8^{\prime} \times 10^{\prime}$ in size. The dimensions of the selection and retrieval portion of the system will be $4^{\prime} \times 3^{\prime} \times 4.5^{\prime}$ and $4^{\prime} \times 2^{\prime} \times 6^{\prime}$. Other details, including costs, are not as yet available. However, total cost of the prototype model is expected to be about $\$ 75,000$.

\section{2 Chip or Discrete Unit Record Systems}

The second category of document retrieval devices producing replica copies as listed in Table $I$ is that of operating systems or proposed machines which store information items on small pieces or chips of film, together with their appropriate selection codes. These are frequently referred to as discrete unit record systems.

3.2.1 Micro Research System $1 /$

The first of the systems utilizing chips as discrete unit records for which there is an early report of actual operational use is that of

1/ Refs. 53, 215, 216, 217 , and 218 . 
the Micro Research Cards of the Petroleum Research Corporation, previously mentioned in the section covering the historical background $1 /$ of such devices. Manipulation and selection in this system are by semi-automatic means, but are carried out on the basis of integrally recorded indexing information. The output product is the photographic replica of the original item.

As previously noted, the system for the Rocky Mountains Geology Library was in actual operation at least as early as 1956. It is understood that, at present, over 100 Micro Research Card units are in use for various applications. Storage medium characteristics, input and search procedures, and operational details other than those previously mentioned are therefore discussed below.

The cards are of $5^{\prime \prime} \times 8^{\prime \prime}$ dimensions, produced on contrast process ortho film. The document area $\left(5^{\prime \prime} \times 4.5^{\prime \prime}\right)$ can accommodate images of one large item or of up to 56 letter-sized pages as required. The reduction ratios used are either 18:1 or 20:1. The original documents are first photographed with $16 \mathrm{~mm}$ or $35 \mathrm{~mm}$ cameras. These photographic images are then transferred to the cards by a step and repeat camera. The selection code area is a space $5^{\prime \prime} \times 3^{\prime \prime}$ at the bottom of the photographic card. There are 26 holes across the card in each of eight rows. These holes are either elongated or not to provide the required binary code pattern. Thus there is a total selection code capacity of 208 bits. A sample card is shown in Figure 11 .

Input of code information is performed by marking a paper master with an electrographic pencil. This master, mounted on a drum device, is matched to the photographic card, and a special machine is activated to cut slots for the appropriate holes. Subsequent search is carried out by the proper placing of needles in a mechanical sorting box which may hold up to 400 cards for each sorting pass. Search is therefore parallel for the items held in the sorting box at one time. Selected cards are removed from the deck by hand for viewing, contact printing, or printing of hard copies at $24 \mathrm{x}$ enlargement.

The principal equipment costs in a Micro Research System installation would be incurred in a combination camera and automatic high-speed slotter, which for a first model might cost as much as $\$ 25,000$. Manual or less automatic means for slotting, however, cost considerably less, e.g., about $\$ 5,000$ including a suitable camera. The cost of sorting boxes and needles is about $\$ 50$ per unit. System costs in actual operation for the recording of individual items,

See pp. 30 and 31 of this report. 


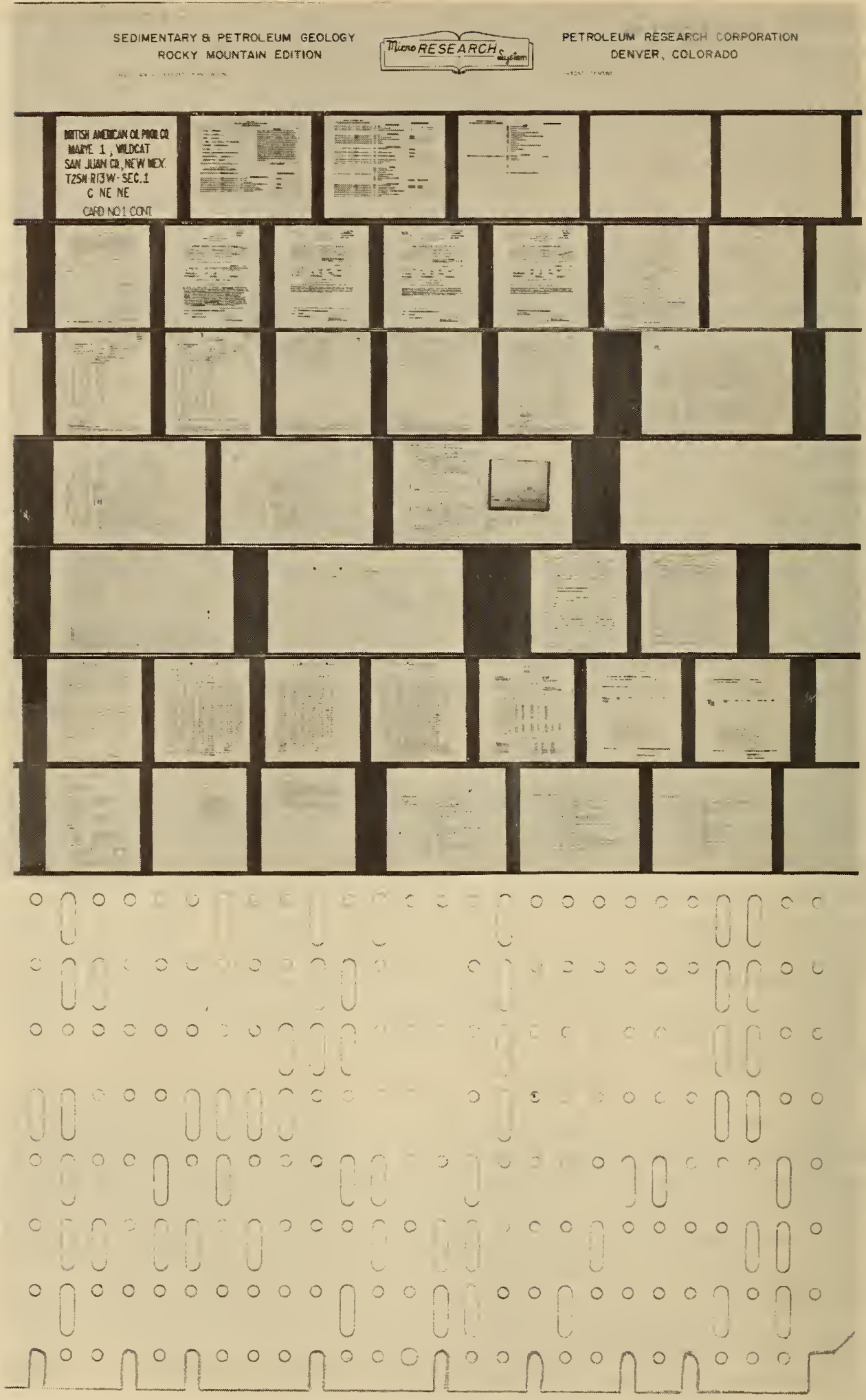

Figure 11. Micro Research Card 
including indexing as well as encoding, are reported to average $\$ 15$ per item. Duplicate cards cost $\$ 0.26$ each, and hard copy prints cost approximately $\$ 0.08$. Since search speed is less than three minutes for each group or batch of 200 to 400 items, each of which may be a multi-page document, the Micro Research System would appear to offer certain advantages, especially for relatively small collections.

\section{2 .2 Filmorex $1 /$}

The Filmorex systems were developed in the period of the early and middle $1950^{\prime} \mathrm{s}$ by Jacques Samain in France. They utilize film chips with space for recording two 8. 5" $\times 11^{\prime \prime}$ document pages plus a selection code area of 400 bits capacity, arranged in 20 rows of 20 bits or five hexadecimal characters each, as shown in Figure 12. By special codes and fixed field format combinations, a number of indexing criteria may be recorded. Several 2 of these systems are in use today, notably an installation at the Centre National de Recherche Scientifique de France and an installation at the Rome Air Development Center, Griffis Air Force Base, New York.

The film chips used as the basic storage medium are transparent microfilm cards, $35 \mathrm{~mm} \times 60 \mathrm{~mm}$, of $8 \mathrm{mil}$ thickness to provide necessary sturdiness in both manual and machine manipulation. These cards are usually cut from coated "Orthochromatic de Securite Microfile" film made by the Eastman Kodak plant in Paris, which was especially designed for use with Filmorex equipment. A film card size of $45 \mathrm{~mm} \times 72 \mathrm{~mm}$ has also been reported for Filmorex systems, and the RADC installation is investigating the possibilities of using Du Pont "chronar" film, which is a polyester base film 5 mils thick. The thickness of the storage medium assumes considerable importance in the Filmorex system because the chips are manipulated mechanically by an oscillating hammer in the Selector unit.

The Filmorex camera and input unit are shown in Figure 13. In the input operations, the operator first places the document itself in position. He draws from a file of vocabulary code cards those cards which correspond to the codes indicated as appropriate for that document by the subject matter analyst. These are stacked one on top of the other so that, for all except the top card, only the blackwhite representation of the code pattern shows. The top card, however, carries the name or code of the descriptor or indexing term in humanly legible form. Document and selection code are then

\footnotetext{
1/ Refs. 90, 248, 249 and 250 .
}

2/ Twelve in France, two in England, one in USSR, and one in USA. 


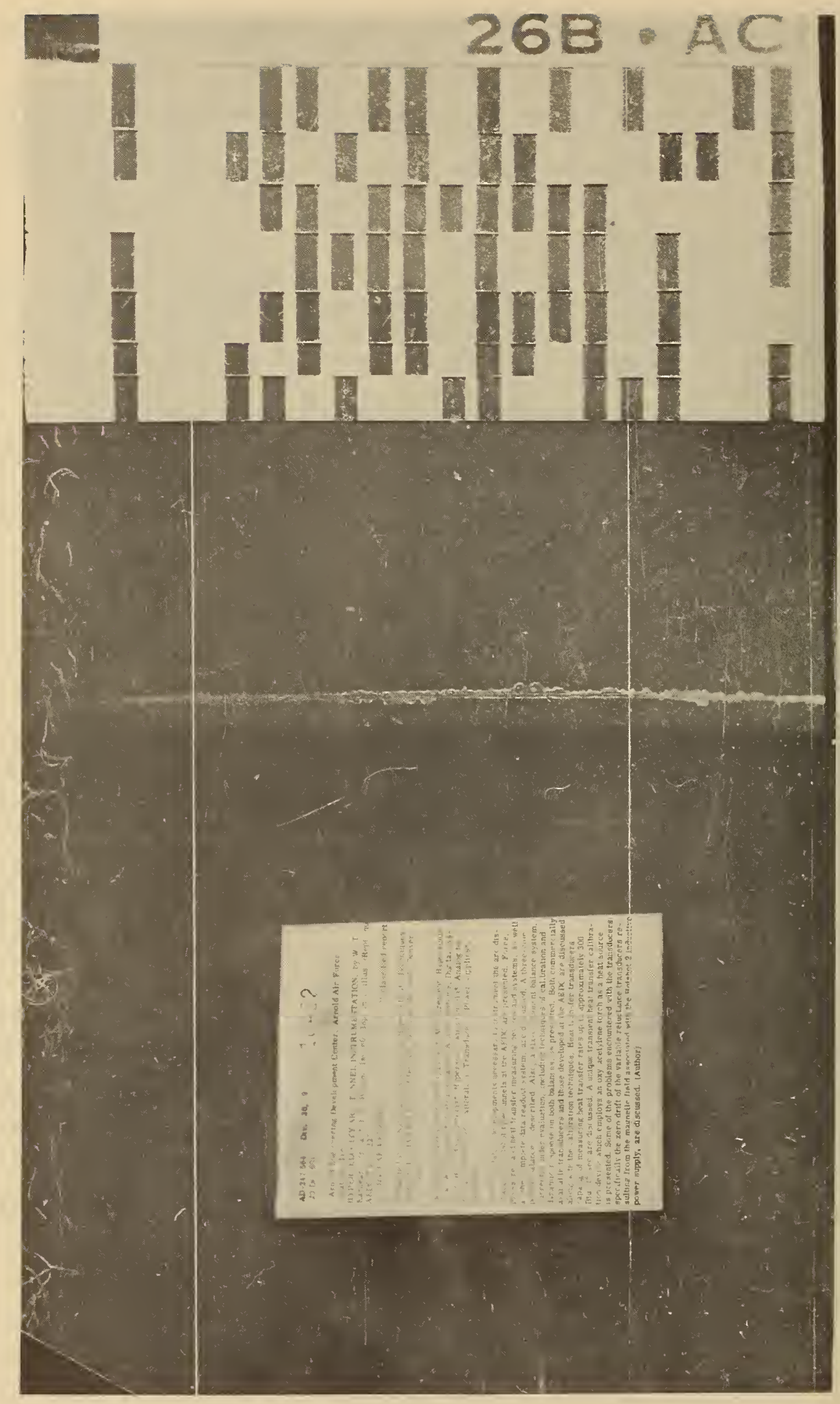

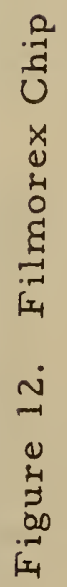




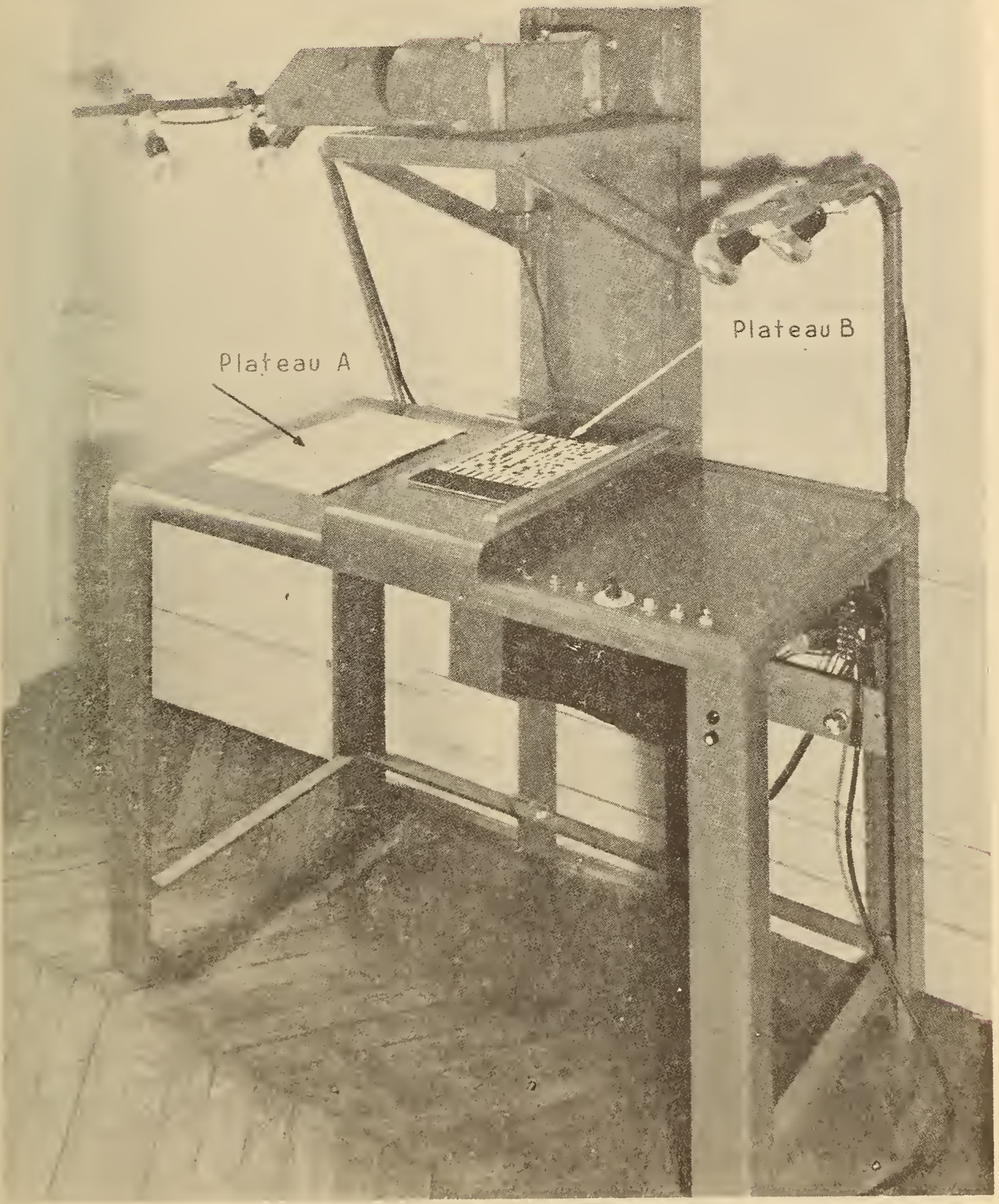

Figure 13. Filmorex Camera 
photographed simultaneously. If desired for compartmentalized multiple filing, the operator may permute the order of code cards in the stack. He then rephotographs the document and the stack so that each additional chip will have a different key descriptor which can be read directly for purposes of manual sorting, filing, and selection.

The Selector unit of the Filmorex systems is shown in Figure 14 . It has dimensions of $2^{\prime} \times 2^{\prime} \times 4^{\prime}$. Photocell scanning and electronic selection circuitry are employed. A perforated aluminum mask, called a search key or scanning grid, is prepared by key punch to give the complement of the code patterns desired. Logical manipulations desired, including "yes", "no", "and", and "or", are set by switches. However, in any given search pass the search prescription can not be allowed to call for more than three patterns. This limitation relates to the desirability of multiple filing so that there are separate decks of chips filed under various leading descriptors. Then, the operator may manually select a deck corresponding to one of the search criteria prior to machine sorting and further selection.

The search rate per pass is 600 to 700 chips per minute. Output is the selected chip itself which can be read in a suitable viewer or which can be projected to prepare either a microfilm copy or an enlarged print through a viewer-reproducer. Where the threepattern limitation on the search prescription prevents completion of a search on one pass, the stacks of tentatively selected items are reinserted for further sorting. These searches are serial by chip, but parallel with respect to the code patterns.

At the RADC installation, operational experience indicates that up to 300 items can be recorded per day. These are presently items from a library of ASTIA abstracts, and those ASTIA descriptors which are starred are used for the selection criteria. The Filmorex equipment was originally chosen because of its simplicity and low cost (i.e., for the equipment, a quoted price of $\$ 7,500$ FOB Paris, France). It was installed in January, 1960.

Difficulties have arisen both with respect to the equipment (film curl and film chip feed jamming) and with respect to the intellectual problems of analysis and adequacy of the indexing system. The equipment problems can apparently be solved. Basic questions as to the adequacy of the ASTIA descriptors for RADC purposes and the possibilities for a "customized" RADC indexing scheme remain under investigation. Nevertheless, a number of advantages are claimed by RADC personnel for their Filmorex system. These specifically include high storage capacity, the inclusion of both graphic and digital material in the same discrete unit record, and the re-sorting, re-arranging, compartmentalized processing advantages of punched card-type manipulation. The low cost of the system is emphasized. 


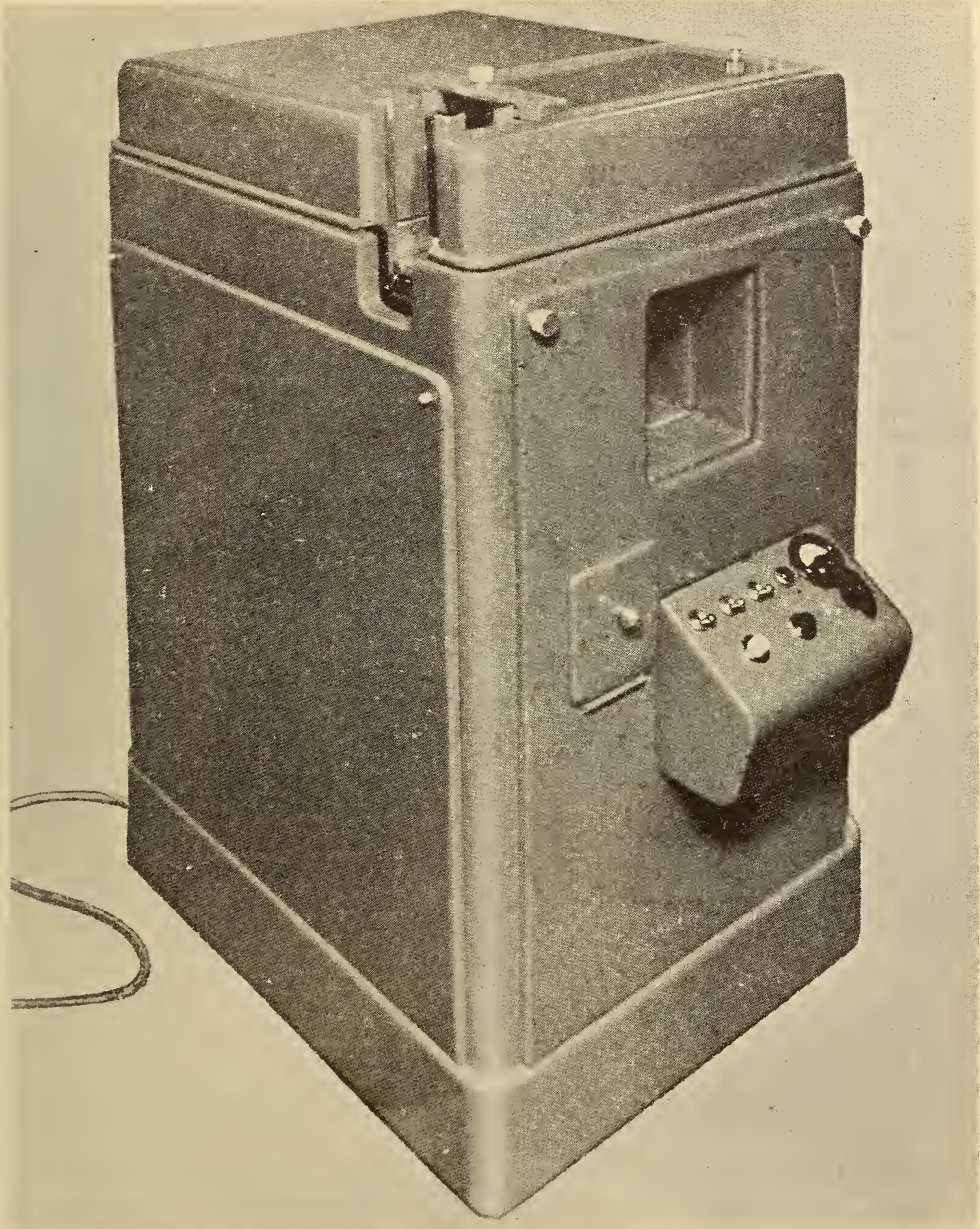

Figure 14. Filmorex Selector 


\subsection{Minicard $1 /$}

The Minicard systems of today derive, as we have previously noted, from early work (i.e., late 1930's or before) at Eastman Kodak in the combination of microfilm storage with mechanical manipulation and selection techniques. In 1953, a proposal to exploit the Minicard development was made to the U.S. Air Force. In 1954, a contract was entered into between Eastman Kodak and the Rome Air Development Center for a Minicard information and intelligence data handling system. This work, described by A. W. Tyler, W. L. Myers, and J.W. Kuipers, 2 ultimately resulted in the first operational system, which had its first public demonstration November 17-21, 1958, on the occasion of the International Conference on Scientific Information held in Washingtón, D. C. $3 /$ There are now at least four Minicard systems in use, three in agencies of the U.S. Government and one at the Eastman Kodak Company itself. 4

Camera, selector-sorter, computer-duplicator, and processor for a typical Minicard system are shown, respectively, in Figures 15-18. In addition, film processors, film cutters, viewers, enlargers, print processors, filing sorters, and other auxiliary devices are typically required. It is noted that this is a very-large-scale system.

Costs of a complete system will, of course, vary with the number of constituent units required for a particular application, but in general the costs for Minicard systems are likely to be comparable to those for large general-purpose computer installations. It should be remarked, however, that the initial application of Minicards was for a very large collection, including, for example, a subcollection of several million aerial photographs. Moreover, multiple-copy filing, high speed selection and processing, and highquality reproduction of replica copies were included in these initial operating requirements.

Refs. 75, 77, 78, 81, 154, 181, 189, 192 and 282 .

2/ Ref. 282 .

3/ "Eastman Kodak demonstrates high-speed Minicard system", Ref. 78 .

4/ A Minicard system designated AN/GSQ-26, also for the Rome Air Development Center, uses a computer-type selector system that operates on magnetic tape item recordings rather than Minicards as the main store or file, and thus lacks the true graphic replica feature. 


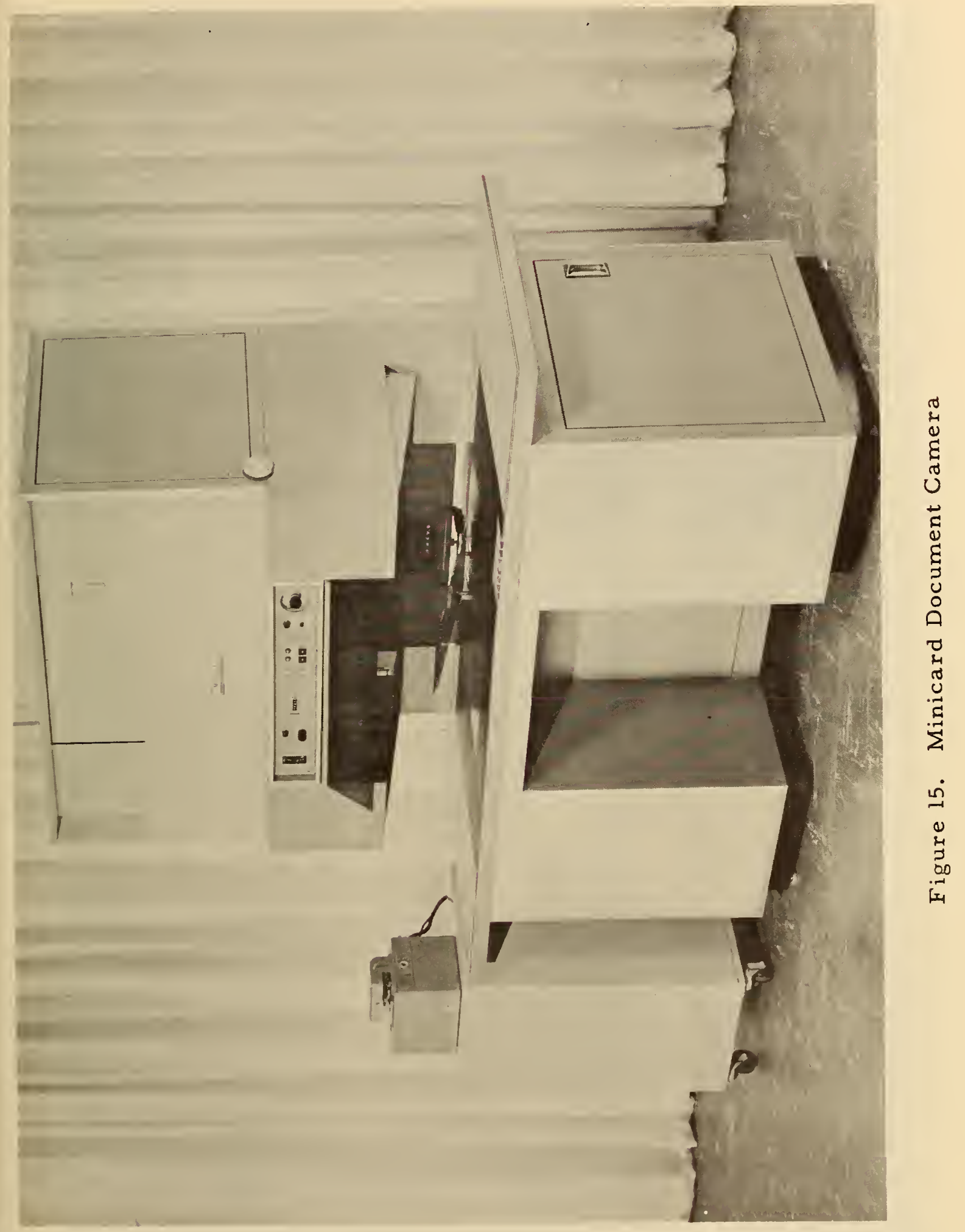




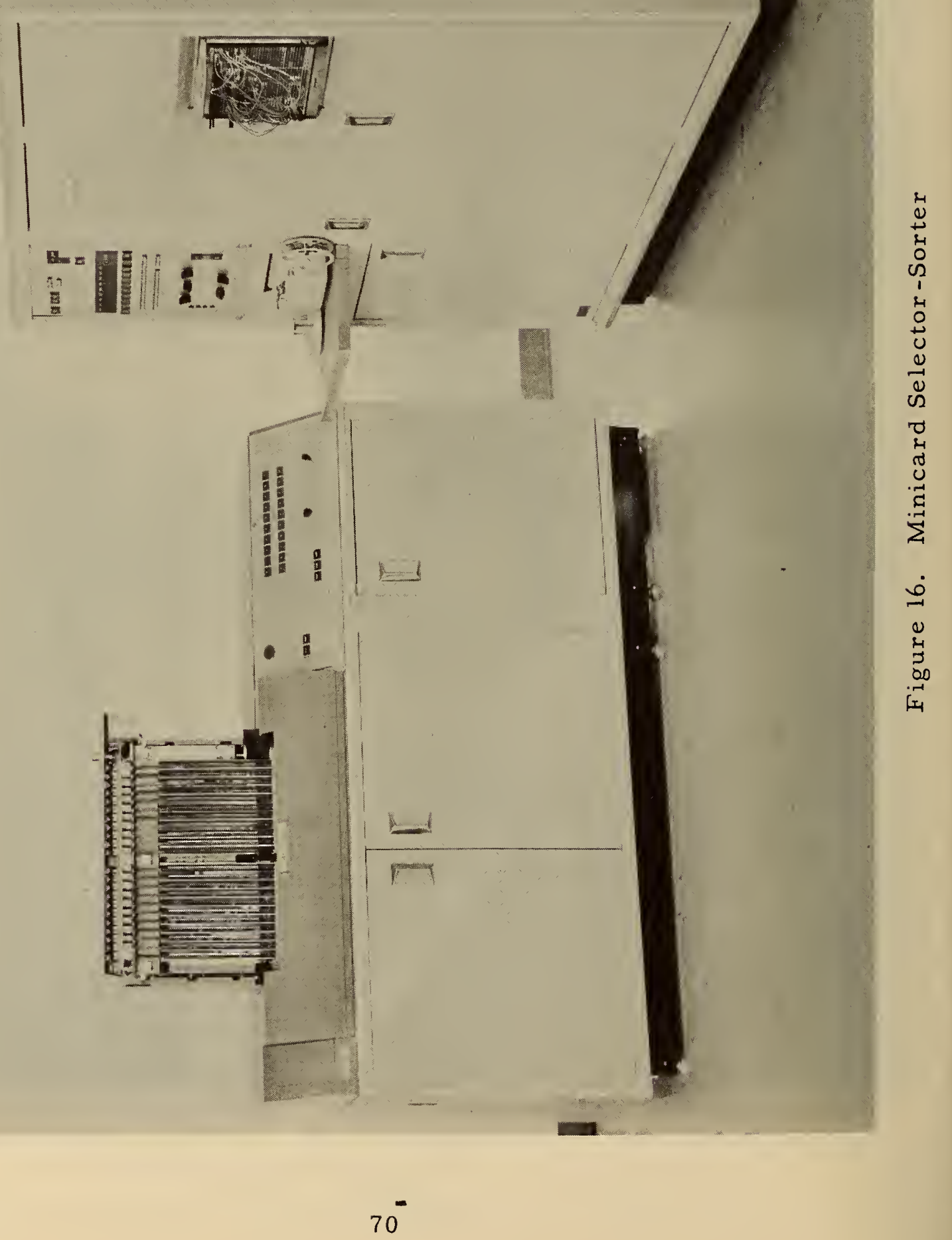




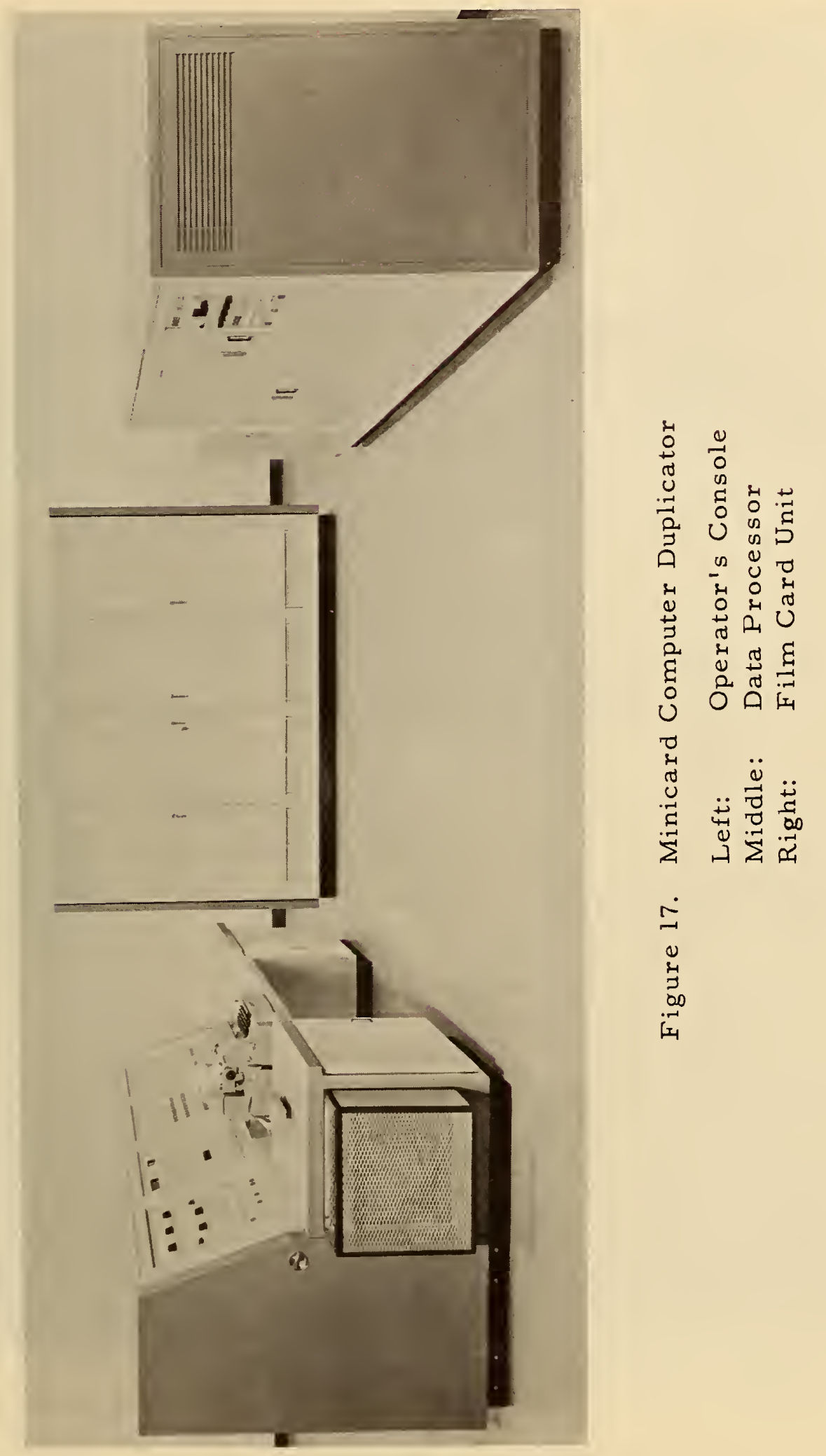




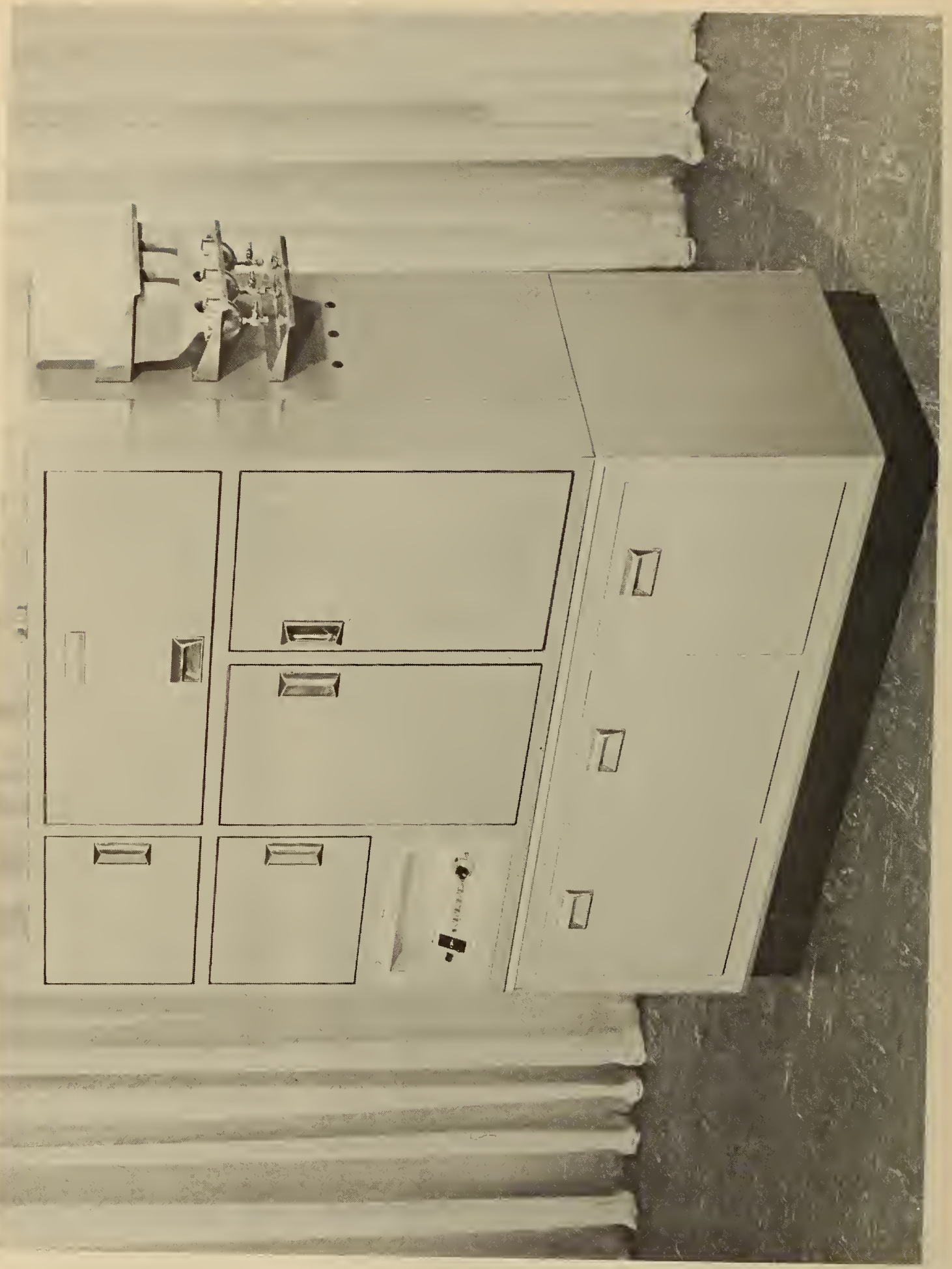

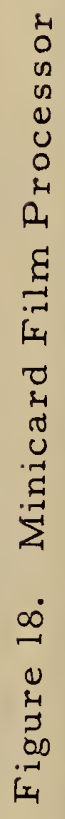


The basic storage medium for Minicard systems is a special fine-grain emulsion film on triacetate base $16 \times 32 \mathrm{~mm}$ in area when cut to the Minicard chip size. Under certain conditions, resolution may be as high as 1,500 lines per $\mathrm{mm}$, and the reduction ratio most commonly used is 60 to 1 . I On each chip, the document and selection code areas can be assigned several variable formats, ranging from, effectively, almost all code area up to 12 letter-sized page images plus a minimum code space for sorting and control purposes (i.e., limitation to address-type selections). The variable selection code format reserves two 42 -bit columns for the sorting field (these two columns remain blank in the master card for each item, but code patterns may be added to replica cards in duplicating operations). Several columns are also reserved as a control field containing such information as document number and the number of Minicards to be treated as a group for filing and processing. The remaining columns, up to a maximum of 7042 -bit columns in all, are used as an openended selection code field. Figure 19 shows the size and format of a Minicard. It should be noted that the Minicard contains a slot used for handling.

In the input operations, code is entered either by means of punched paper tape or by direct keyboard operation at the camera station. A master film of negative Minicards is first prepared. From this, positive copies are made and cut to the chip size for use as required, whether in a single file or in various working files. In some applications, Minicard duplicates, one for each significant code inscribed on the master chip, are made for each item. These duplicates are filed in separate sections of the file which are reserved for a particular selection criterion or a particular combination of such criteria. Minicard duplicators may be so used as to provide mechanically for the re-ordering of code patterns and the identification of a "filing-key" code, such as is achieved manually by the operator rearranging the code card stacks in the Filmorex systems. In particular, selected code patterns for filing purposes may be automatically recorded by the duplicator equipment into the sorting field columns left blank on the Minicard Master.

There are both advantages and disadvantages to this approach. Thus, as Eastman Kodak investigators have reported:

"Multiple entries for a file item expand the size of the file and require more duplicating and more infiling. The whole purpose, however, of a multiple-entry approach is to achieve an organization of the file so that all duplicates

1/ Other reduction ratios for large maps, drawings, and photographs may also be achieved by use of specially designed cameras. 


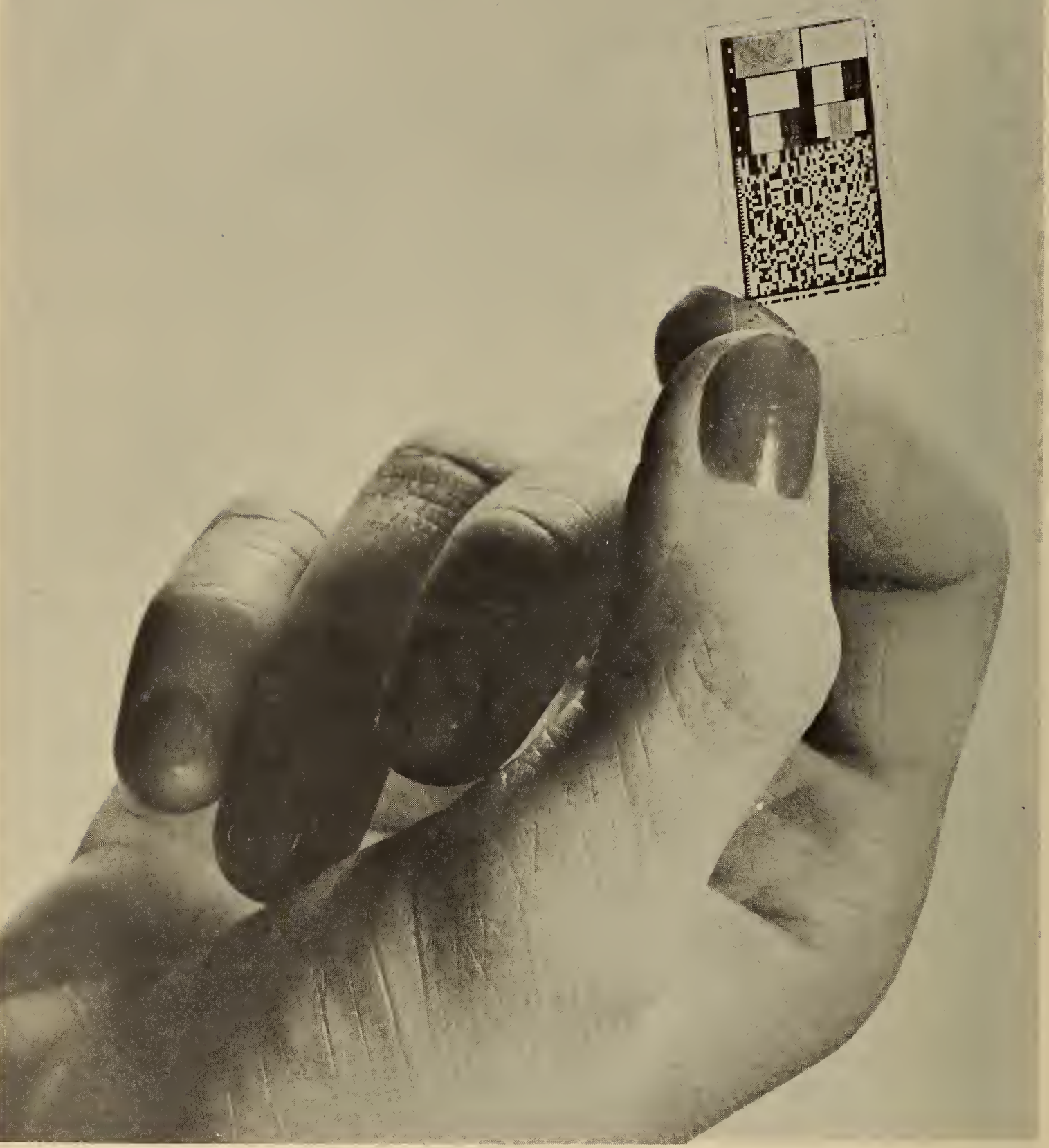

Figure 19. A Minicard 
having to do with a particular code category may be brought together in one section of the file, then, when a search must be made, only a portion of the total file need be presented for the searching operation. " 1 /

In a particular operational application of Minicard equipment, two file sets are used in parallel. In response to a specific inquiry, a search is made against the appropriate section of the positives file. Within 10 minutes at most, the querist will have delivered to him the actual selected item or items from this file. An identical search is then made against the file of masters (negatives), and duplicate copies of the responding items are made to replenish the positives file. These are re-filed automatically by machine techniques.

In general, because most Minicard systems are intended for large-scale high-activity applications, as much of the handling, filing, infiling, and processing as possible is done by mechanical means. Individual Minicards are mounted through the slots in the chips on sticks in "shishkabob" fashion, with a packing density of 2, 000 chips per stick. Sticks in turn are grouped into file "blocks" or magazines, which are combined into file units of, say, 10 or 50 magazines each. For example, a three-drawer file cabinet of dimensions $15^{\prime \prime} \times 30^{\prime \prime} \times 50$ " can accommodate several 50-magazine file units with a total capacity of 900,000 Minicard chips representing up to $11,000,000$ coded document pages in microform. However, 2,000-item groups of these records can be removed manually by means of the skewer-sticks.

With regard to the selector-sorter equipment, searches are set up by punching tape and by setting up the appropriate logical relationships desired -- "and", "or", "greater than", "less than" -- on a program logic plugboard. Searches are serial with respect to chips read in sequential order and with respect to the rows of the selection code area, but parallel with respect to each row. The output of a search may be a duplicate Minicard, an enlarged hard-copy print, or a microfilm copy mounted in a $3^{\prime \prime} \times 5^{\prime \prime}$ aperture card. When the duplicates of selected items have been made, they are combined with the non-selected records and returned to the file as soon as possible.

Operating rates that have been reported for various phases of Minicard system processing include the following:

(1) Input recording rates for average documents (i.e., 200 characters of selection code, six document pages) -40 to 90 per hour, per camera station.

(2) Minicard duplication rate -- 120 Minicards per minute.

Kuipers, J.W., A.W. Tyler and W. L. Myers.

"A Minicard system for documentary information, " Ref. 154, p. 258. 
Aperture card output mounting -- 10 Minicard records per minute.

Enlarger-print processor rate -- 480 prints per hour.

Search rate in the 1959 model Selector-Sorter -- 1, 200 Minicards per minute.

It is to be noted that search time in a Minicard system depends on the extent of compartmentalization and subject or selection category segregation that can be achieved in the organization of the files. Thus, for very large files a search of the entire file might take a matter of hours, whereas the same collection maintained as a group of multiple-entry working files might require a search time of a few minutes. The maximum time to locate an individual item on a particular Minicard stick is 1.5 minutes. Under operating conditions, an average time of 10 minutes per search, from input of the search prescription to delivery of selected chips for viewing or reproduction, is not uncommon.

\section{2.4 Magnavue $1 /$}

The Magnavue System, which uses a magnetic aperture card for document and code storage, is currently under development by the Magnavox Corporation for the Rome Air Development Center. A laboratory model of this system has been tested using a prototype model of the Magnacard 2 equipment, to which a visual image display device has been added. This model is currently undergoing modifications.

There are two different storage media used in Magnavue. For the documents, DuPont 4696 photographic film on mylar base is used to record a single page image in a $7 / 8^{\prime \prime} \times 7 / 8$ " area. Reduction ratios used vary from $10: 1$ to $30: 1$. Selection code information is recorded magnetically on a mylar base card. The two parts are bonded together by a lamination process, with the photographic emulsion and the magnetic surfaces placed face to face so that

1/ Refs. 42, 101, 117, 121, 167, 200, 202, 278 and 299.

2/ A Magnacard System is a storage and retrieval device also developed by Magnavox. It has not been separately included in the present report because it does not provide graphic facsimile copies as output. It is similar in principle of operation to standard EAM equipment. However, the cards are coated with a magnetic material on which information to be stored and selected is recorded in digital form. 
the bases form a protective coating for both recording surfaces. The selection code area is $1^{\prime \prime} \times 2^{\prime \prime}$, providing space for 2,700 bits of code information.

Packing density for the magnetic recording of code is 100 bits to the inch, recorded in 20 channels, two of which are used for timing signals and parity checking. The code format uses 18 of these channels to give three 6-bit, alphanumeric characters per column. There are 150 such columns per card. Because the storage medium used for the selection code is erasable and reuseable, additions, changes, and corrections can readily be made. The storage life of the magnetic card is estimated to be 20, 000 machine passes.

Handling units, equipment, and procedures for the Magnavue System are substantially those found in the Magnacard System, except that visual display and replica copying of the selected items is provided. This system therefore includes a Magnascriber keyboard input device, a means for completely automatic handling of the chips after they have been cut to proper size and stacked into magazines, a card transport system consisting of pneumatic vacuum drums, a feed-stack station, a transfer assembly, a data processor, a datacontrol unit and a new image display station.

Magazines of the Magnavue System hold 3,000 chips. A vertical file block holds 10 magazines at a time, or 30,000 chips. A file unit consists of 15 such file blocks (150 magazines or 450,000 chips) and a mechanism to insert or extract any magazine from a vertical file block into the card transport feed-stack station. The average time required to remove a file block from the station and locate and insert a new block is approximately 15 seconds. The average access time to a particular magazine in the block is an additional 3.84 seconds.

The magnetic card transport unit uses vacuum drums as the basic means of moving and manipulating cards. With each drum there is associated a feed-stack station which releases cards onto the drum or carries out the re-stacking of cards picked up from the drum at a rate of 90 cards per second. The transfer assembly enables the transfer of cards from one drum to another. The release of cards onto or from a particular drum, as well as reading, writing, and selective transfer, is accomplished by the data control unit. The data that are written or read are stored in the data processor as are the instructions for the data control unit and the search requirements.

The proposed operations of Magnavue Systems, when used for document retrieval, begin with photographing the document image page by page. It is assumed that each document has an accession or other identifying number assigned. For the completed magazine of cards with document pages recorded, each card is visually displayed to a Magnascriber keyboard operator who copies the document number into the selection code area. These cards are then restacked. The selection codes assigned to the document are also 
recorded by Magnascriber onto separate Magnacards. The two sets of cards are then read together on the card transport unit and collated by document number to produce a new, complete, coded item card.

Selection and retrieval operations may be either of the addresstype on document number or of the search-type. In the latter case, logical manipulations can be provided by the data processor that is associated with the system. Provision is also made for a two-step process on which the actual search would be made on Magnacards (without images) to give document numbers, followed directly by an internal look-up operation to select the corresponding image-carrying cards. Output may either be a visual display or a hard-copy print. A 10-sided drum is proposed for either on-line or off-line stationary viewing or printing. An image superimposition device, shown in Figure 20, has also been proposed. This would involve a display wheel carrying 10 different image cards which belong to the same "family" or set of, for example, maps or charts. These images would then be partially or wholly superimposed so that the user might detect changes or trends.

\subsection{5 "Is There a Doctor in the House?" 1/}

Ideas for a parallel search system for document retrieval, using film for document image storage and interrogating all items simultaneously, were advanced in a report of the Benson 7 Lehner Corporation prepared for the Office of Naval Research.- 7 This proposal can best be described by an analog to situations in which the question "Is there a doctor in the house?" is asked, and any doctor who is present immediately responds. The proposal suggests the incorporation of two features into large file systems usually found only in small files. These features would (1) permit entering the file for simultaneous search in parallel and (2) enable the operator to monitor the selections being made to the extent that he may continuously inspect the number of documents found to satisfy each part of the question.

The system would operate in a similar fashion to that found in edge-notched card systems using needle sorting boxes except that coded light pipes would be used. Visible indications of recognitions made would be presented to the user. As the user enters various selection terms into a keyboard, the effects of each selection criterion and of the criteria combinations are immediately available.

1/ "A study of the fundamentals of information storage and retrieval", Ref. 303; also Taube, M. and H. Wooster, "Information Storage and Retrieval", Ref. 273, pp. 188-189.

2/ Ref. 303 . 


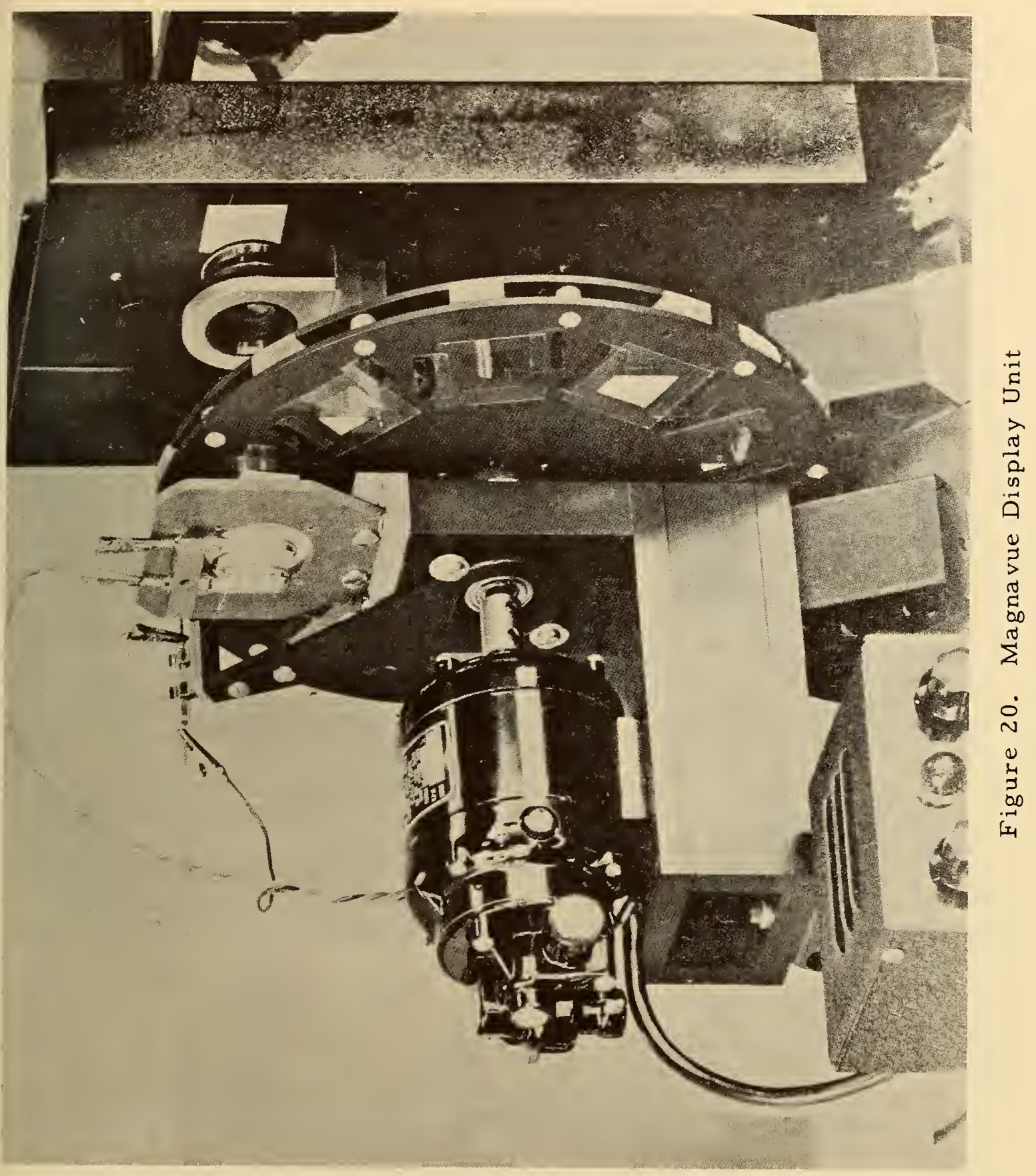


It is assumed that approximately 20 searches per hour could be made per file rack, each of which store 75,000 items.

It is further assumed that each item will have a format such as that shown in Figure 21, where the terminology "the reference" is equivalent to what we have termed the "selection code" and where the individual elements of this reference, called "cells", may take on several possible states. A possible implementation for this scheme is described in part as follows:

"... Each document remains in position during the interrogation process. ...

"... the question must be brought to the item, wherever it may be in the store. ... One method appears to best meet our requirements. This is a piped-light communication channel using plastic or glass plates, the outside of which would be opaqued and made reflective so that light travels certain paths without dispersion. ...

"Under appropriate circumstances, light could leave the communication channel and travel towards a cell. The cell, having several functional stages... must as the first stage have some means of receiving the light. Then facilities must exist so that the light can trigger a series of other functions within the item which as an integration make the item respond. ...

"If from the total spectrum of light we select only two different colors, for instance yellow and blue, we can produce band-pass filters for those wavelengths... . ... If we provide the cell with either a yellow or blue band-pass filter we can consider the cell having either of two states $S_{1}$ or $S_{2}$ respectively. ... The (corresponding) questions ... are now:

\section{a. Are you $S_{1}$ ? \\ b. Are you $S_{2}$ ?}

A cell can answer to these questions by letting light pass or not pass. ...

"... We require the following from the circuit: $1 /$ It must be a conductive line leading to some kind of visual indicator. It must connect a number of 'switch-like elements'. It must contain an energy source or equivalent. The circuit and its elements may not have greater bulk than the item itself. ...

I. e., internal circuitry associated with each item. 


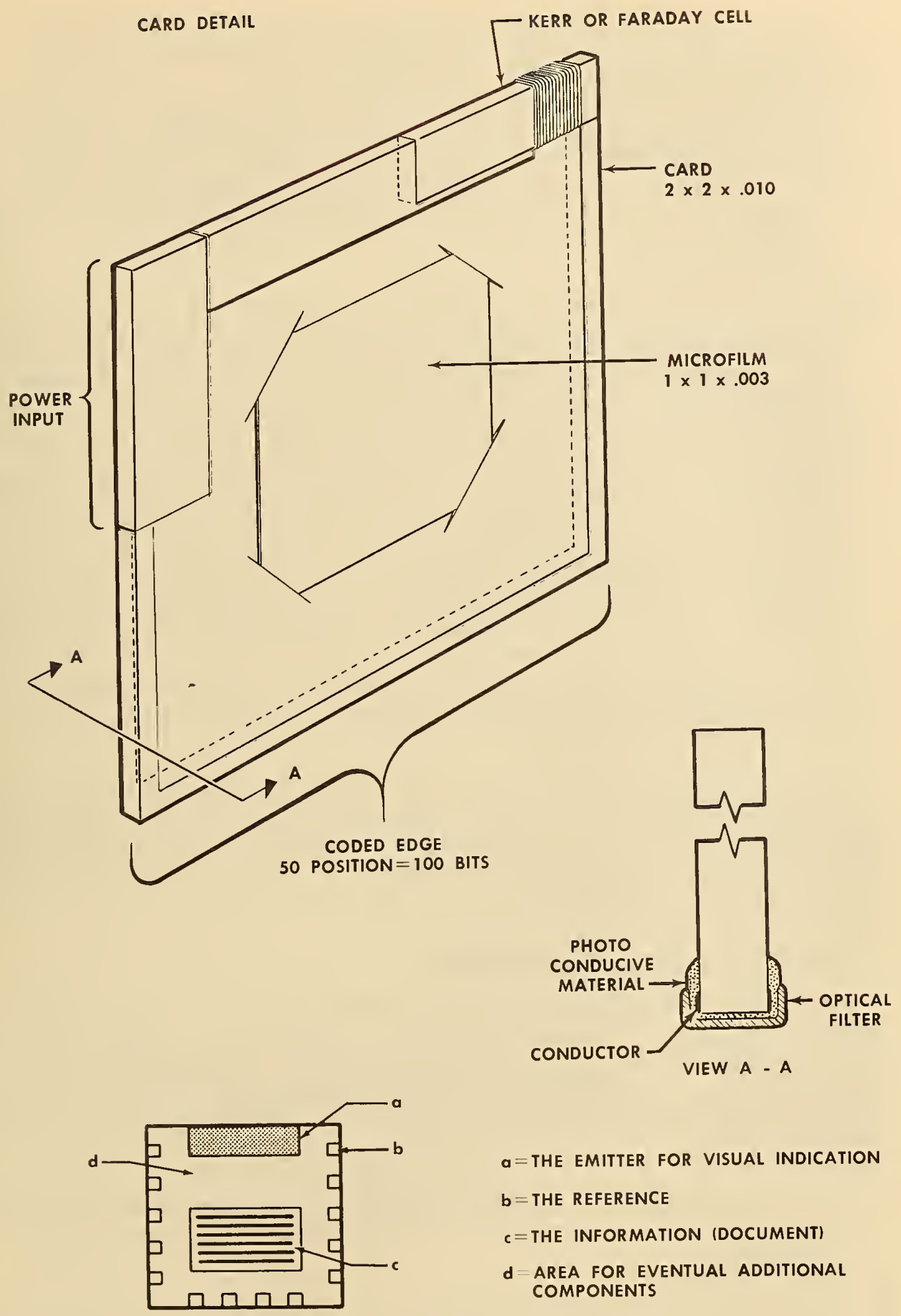

Figure 21. Data Bank Format 
"Considering a circuit with a number of parallel resistors, we can make the resistors photosensitive and place one such resistor behind each filter. ... then any light passing the filters will lower the resistance (effectively causing a short-circuit) and produce a flow of energy through the circuit. This is the case if ANY filter (cell) transmits light. Since a reference matches if all cells match, 17 but mismatches if only ONE cell mismatches, this system would result in a mismatch indication which would require a complementary coding. ...

"The following solution was found for a visual indication. A Kerr cell (using the Kerr magneto-optical effect) has been developed for evaporated circuitry, where a layer of appropriate material is evaporated onto a surface. This system is surrounded by evaporated wires forming a coil. If current flows through this coil, its magnetic field will change the properties of the layer so that plane polarized monochromatic reflected light will suffer a rotation. If such a cell is offered monochromatic polarized light, it will then in its working state rotate the plane of polarization further so that through an analyzer this cell and the item to which it is attached appear darker. A permanent magnetic foil behind the Kerr cell acts as a bias returning the cell to a normal state on the removal of the current. Since all mismatching items are darker the visual indication for matches would be a reflection, that is, a bright appearing item. ..." 2 /

Possibilities for storing the items incassettes, (and these in turn are stored in $4^{\prime} \times 4^{\prime} \times 4^{\prime}$ racks containing 75,000 items, all of which could thus be searched simultaneously), and for using superimposed coding for coordinate indexing in the interrogation process have also been considered.

\section{3 Sheet or Strip Film Systems}

In addition to the continuous roll film and film chip systems as used for document retrieval devices, various compromises have been suggested. These are usually intended to gain some of the advantages and eliminate some of the disadvantages of the other two systems. For such purposes, sheets or strips of film have been

1/ I. e., if the selection code pattern for the item exactly matches the code pattern required to be satisfied by the search prescription.

2/ Weber, E. "A retrieval solution", appearing in Benson and Lehner on "A study of the fundamentals of information storage and retrieval, "Ref. 303. See also Weber, E. "A suggestion for an improved data banking system", AD 225 097, Ref. 293. 
proposed for use with automatic selection mechanisms. With the exception of Microcite, those that are in actual operation today are primarily address-type selection systems. However, in some cases, current system design planning is directly concerned with possibilities for search-type modifications. Of these systems, those which are representative of address-type systems potentially adaptable for subject searches will also be discussed.

\subsubsection{Microcite $1 /$}

The Microcite equipment, developed and operated experimentally at the National Bureau of Standards, $j$ s shown in Figure 22. It is an extension of a Peek-a-boo system. 2 / Peek-a-boo systems utilize Batten, term entry, aspect, or index term cards to index literature for later search; i.e., internal hole locations that identify document numbers are punched into just those index term cards that represent index terms specifically applicable to the document. In searching, the cards corresponding to the terms of the search prescription are located and visually compared for hole coincidence. Whenever this occurs; the document indicated meets the index parameters of the search. The output of such systems is a list of document numbers, and a separate retrieval operation is required to select the corresponding documents from file.

Microcite provides a Peek-a-boo system with a means for visual display and output copy of the selected document's abstract. These abstracts are stored as micro-images on a film matrix. "In operation, the film matrix appropriate to the peek-a-boo set being searched is selected and mounted on a drum. The operator places selected peek-a-boo cards on an illuminated area of the machine and manipulates two control wheels to position a set of cursors on each illuminated, that is, unobscured hole in turn. Turning the control wheels causes rotation and translation of the drum carrying the film matrix, so that as the cursor is set on each hole, a full-size focused image of the citation and abstract corresponding to that hole is projected on a screen in front of the searcher." $3 /$

Posting document numbers in a Peek-a-boo system involves locating, in a rectangular array on an index term card, the coordinates corresponding to the document number and punching a hole at that point on each card applicable to the document. A search will then let light pass through the same hole locations in several stacked

$1 /$

2/

3/

A new Microcite machine", Ref. 206

"Microcite, an aid to more effective referencing", Ref. 179

"A new Microcite machine", Ref. 206. 


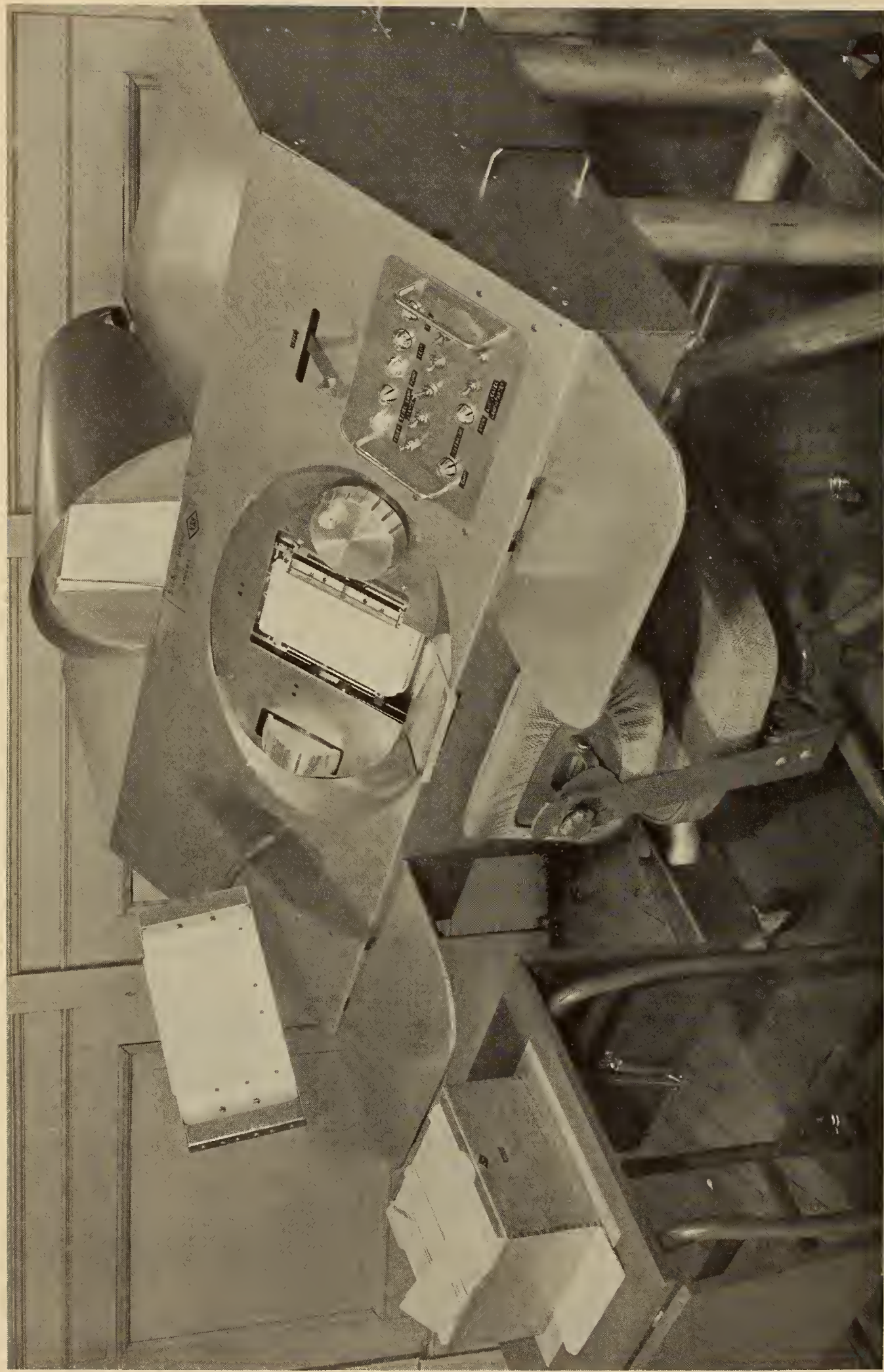


cards indicating, with the aid of a grid, the numbers of those documents indexed by all the terms selected for the search, hence the documents to be retrieved.

In Microcite, the location of each indicated hit is used as a reference point from which to position and project a microfilm image of the abstract of the document for visual inspection by the querist. The searcher may then (1) determine the probable relevance of the projected items to his question prior to retrieval of the full document text, (2) selectively sample the results to ascertain whether his search prescription is too broad or narrow, and, with collateral use of an indexing term dictionary (coding vocabulary), (3) either reformulate his search prescription in the former case or add and/or remove index term cards in the latter. These operations are possible because the searcher becomes part of a closed feedback loop when he is conducting a machine search; that is he receives indications of the machine output from which he may modify the input. In terms of "directed browsing" (where the user poses a deliberately broad question), a convenient tool is thus provided. A degree of "free" browsability is potentially available if consecutive document numbers are assigned for items of similar subject content. The searcher can operate the cursor controls (handwheels) so as to project the abstracts of non-indicated items that are physically adjacent to a selected item.

Microcite is therefore a special-case system involving searchtype selection that not only retrieves address locators, but is coupled with a direct facsimile output of document abstracts (projected image and, if desired, hard copy). It thus uses two basic storage media: viz., film on which the abstracts are copied and Peek-a-boo cards for storage of the document codes. $1 /$ The process for storing the abstracts involves first filming onto $16-\mathrm{mm}$ double perforated microfilm strips and then contact printing to produce the proper array on "ortho A litho", polyester-based film sheets. At a reduction ratio of 37.75 to 1 , there are $18,0003^{\prime \prime} \times 5^{\prime \prime}$ abstracted items recorded in a $15^{\prime \prime} \times 15^{\prime \prime}$ area on a $20^{\prime \prime} \times 24^{\prime \prime} \mathrm{film}$ sheet. Since the Microcite equipment has so far been used only for experimental operations, no data are available as to time and costs for filming abstracts on a production basis.

The Peek-a-boo or search cards presently used are of $5^{\prime \prime} \times 8^{\prime \prime} \mathrm{x}$ $.007-.010^{\prime \prime}$ polyvinyl stock, of which there is one each for every primary index term used in an 18,000 document (sub) collection. For input of selection code information, index term cards applicable to a, specific document are individually and manually drawn and punched 2 /

\footnotetext{
1/ Ref. 179. A third level of storage, whether printed paper or photographic copies, is obviously required for the documents themselves.
}

Ibid. A commercial version of the punch is available. 
for each term assigned to the document. The hole is made at the proper coordinates for the document number. The rate of the selection code inscription operation is approximately that by which 33 documents per hour may be coded.

For a search, the appropriate term records (Peek-a-boo cards) are manually selected and stacked together on a back-illuminated search easel. In manual search, a grid overlay indicates the numbers of selected documents. With Microcite, the cards are placed under cursors over a similar easel in the face of the machine. Then, movement of the handwheels so as to position the cursor intersection over the holes of visual coincidence will simultaneously position a full-size enlargement of the abstract microimage onto a screen so that the indicated item may be readily inspected. Output may be limited to this display or a hard copy may be obtained on Polaroid Land film or oscillograph paper, or the querist may himself record document numbers or other bibliographic citation data in order to retrieve the original documents.

Search is made in parallel for each group of 18,000 documents and for larger collections would be repeated for each such group or file section. 1 In one search, approximately a minute would typically elapse between physically locating the index term cards and projection of the first abstract. About three seconds is required for each succeeding item. However, total search time is obviously affected by the user's inspection or scanning time.

The size of the search and projection equipment is that of a small desk or about $4^{\prime} \times 3^{\prime} \times 4^{\prime}$. Costs are estimated at $\$ 1,100$ for the manual system, which includes the Peek-a-boo card punch and illuminated readout stand, and $\$ 10,000$ to $\$ 15,000$ (using Government machine shop costing) for an exact copy of the present prototype model of the Microcite search-projection equipment.

\subsubsection{Large-Capacity Document Storage and Retrieval} System (IBM-Walnut) Zf

The Advanced Systems Development Division of IBM Research Laboratories, San Jose, California, has developed a large-capacity document storage and retrieval system. This system uses the IBM 1410 computer, a large random-access digital magnetic storage device and a special image file.

1/ Ref. 179. Note that a separate film sheet must be placed in the machine for each set of Peek-a-boo cards. It is planned that this operation may become automatic in a future model.

2/ See Refs. 138 and 223. 
The index or search terms and a brief abstract of the documents are stored in the digital magnetic memory associated with the 1410 . The complete text of each document is stored in the image file. A search is made by feeding into the 1410 the desired search terms and their logical relationship. The computer translates these terms into machine language, makes the search, and delivers cards which are punched with document address codes and onto which are printed the index terms and abstract of the document. The searcher reviews these cards and returns to the system those cards which represent the texts he desires to see in full. The image file then automatically locates the desired documents and copies them onto aperture cards which are delivered to the requestor.

The document images are stored in the image file on Kalvar film strips $0.9^{\prime \prime}$ wide by $15.5^{\prime \prime}$ long. The image area for each $81 / 2^{\prime \prime} \times 14^{\prime \prime}$ document is $0.242^{\prime \prime} \times 0.400^{\prime \prime}$ at 35 to 1 reduction. Under these conditions 99 document pages are recorded per strip. The strips are grouped into "cells", each containing 50 strips, and the cells handled in groups of 200 . Such a group is called a module and contains 990, 000 document pages.

The input operations for storing documents involve, first, a recording onto $35-\mathrm{mm}$ perforated microfilm at reduction ratio of about 16 to 1 and, second, the transfer of these images onto the Kalvar strip at a further reduction so that the total reduction ratio is 35 to 1 .

At the time of the final recording an address is assigned each item. This is also punched into cards containing the corresponding index terms and abstracts. The data from these cards are then stored in the large digital magnetic memory for search and retrieval use.

The Kalvar film used for the image file has an emulsion of 500 lines per $\mathrm{mm}$ on a mylar base. The transfer of the microfilmed images to the image file strips requires about two hours per cell, which is 4,900 document frames. The transferring and address assigning device is shown in Figure 23. Figure 24 shows the image file which is currently undergoing operational tests.

Search time averages about five seconds of computer time per item, including the printout of address data and abstract. Image search and printout time is 5 to 10 seconds for the selected texts delivered to user as aperture cards. Information as to probable cost of the total system is not yet available. 


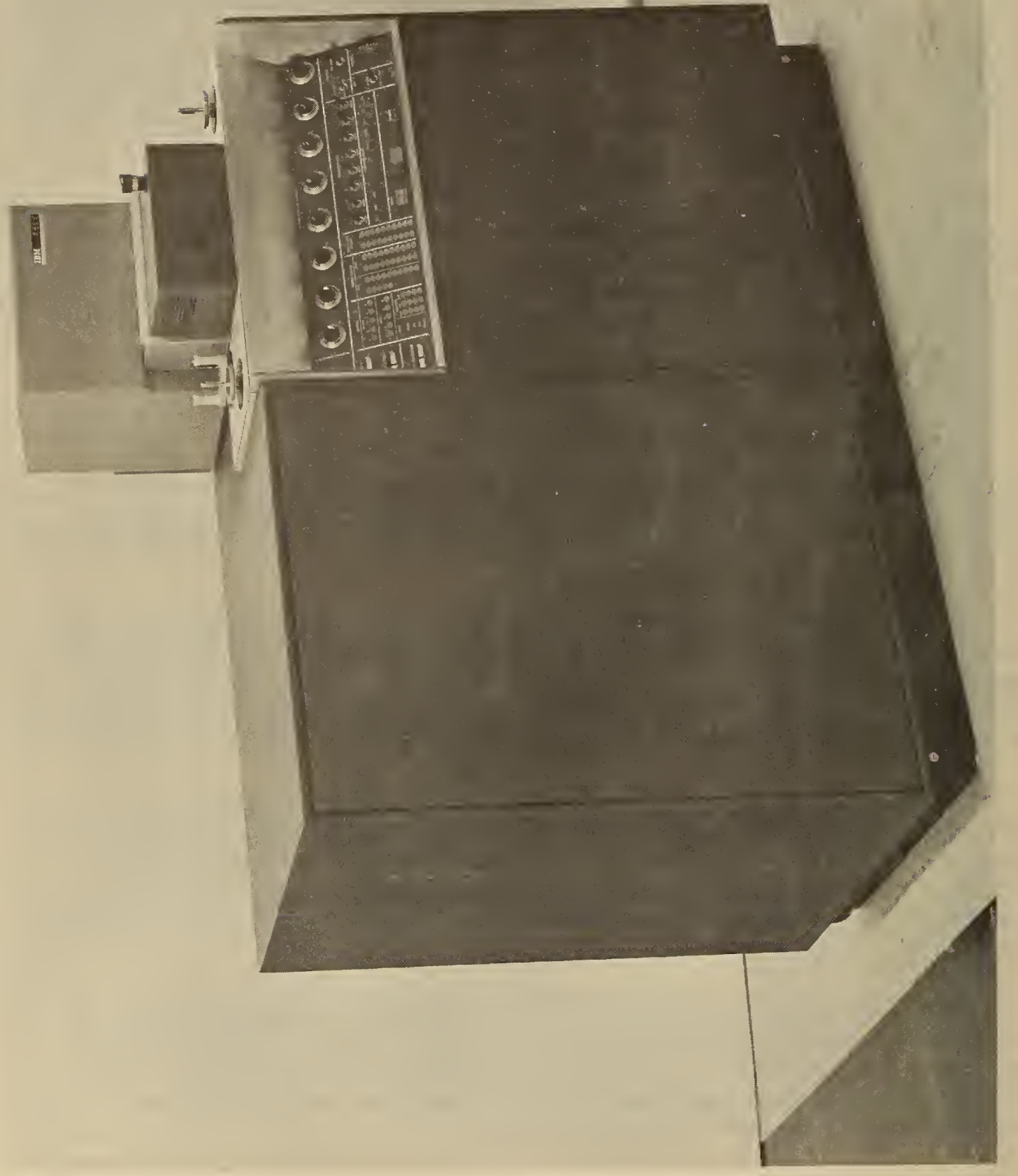

D 


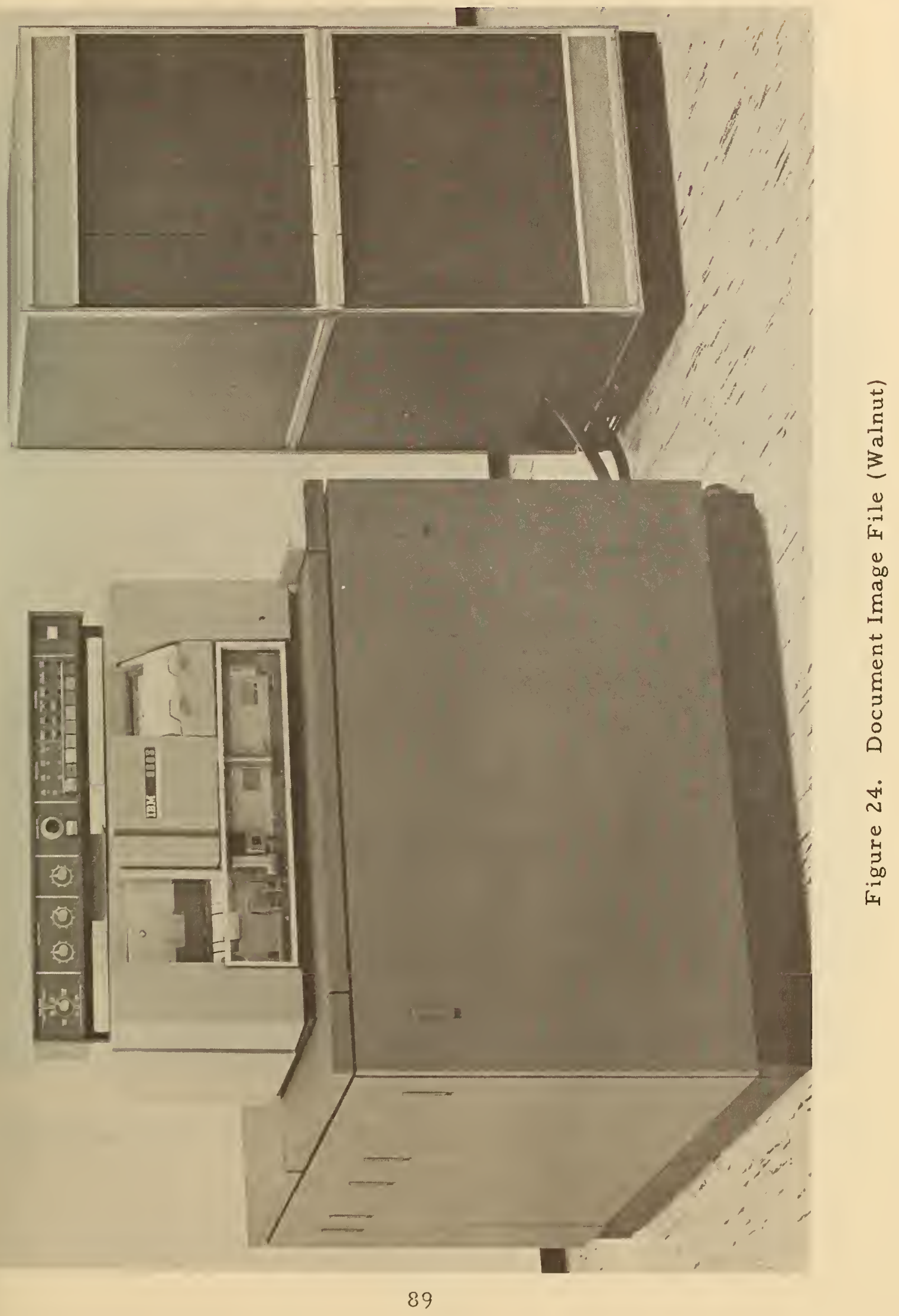




\subsubsection{Documentary Storage and Retrieval System--Staats $1 /$}

The Documentary Storage and Retrieval System exists primarily as a proposal, but a small model of the search machine has been built, as shown in Figure 25. $2 /$ Proposed and developed by Henry $N$. Staats, this system would use electrostatic printing plates (zinc oxide) as the basic storage medium. On each $5^{\prime \prime} \times 8^{\prime \prime}$ plate, up to 180 letter-size pages may be stored as micro-positive images. These images may easily be copied, erased, or replaced.

For input recording a special camera and coding equipment will be required. A reduction ratio of $20: 1$ and a resolution of 10 lines per $\mathrm{mm}$ is expected. The selection code recording is achieved by removing insulation at any of 128 specifiable locations on the upper edge of the image-storage plates. This provides electrical contacts or sensing points for the selection mechanism. Either fixed or variable field coding may be used. Selection is accomplished by activating the appropriate contact wires and passing or "rippling" the storage plates against them. When the activated wires make contact with the image plates matching the search request, these plates are ejected into a rack for viewing or copying. A search speed of 32 plates per second is anticipated. The output product would be microimage prints.

\section{4 Microfilm Aperture Card Systems}

The idea of combining microfilm document image storage with the advantages of card manipulation was, as mentioned in the historical background, an early suggestion for improving literature search techniques. By 1951, in the first edition of the book "Punched Cards, their application to science and industry, "3/ several contributors had seriously considered the use of this technique for scientific information selection and retrieval systems. In 1958 , however, when a second edition of this book was published, only a few examples of operational use were included. The example of Eastman Kodak was cited as follows with respect to possibilities for storing and retrieving photographic negatives:

$1 /$ Staats, H.N. "Description of the Staats documentary storage and retrieval system," Ref. 266.

2/ Glass, rather than metal, plates are used in this representation to illustrate the principles of the system.

$3 /$ Ref. 49, p. 212, Casey, R.S. and Perry, J. W. "Punched Cards". 


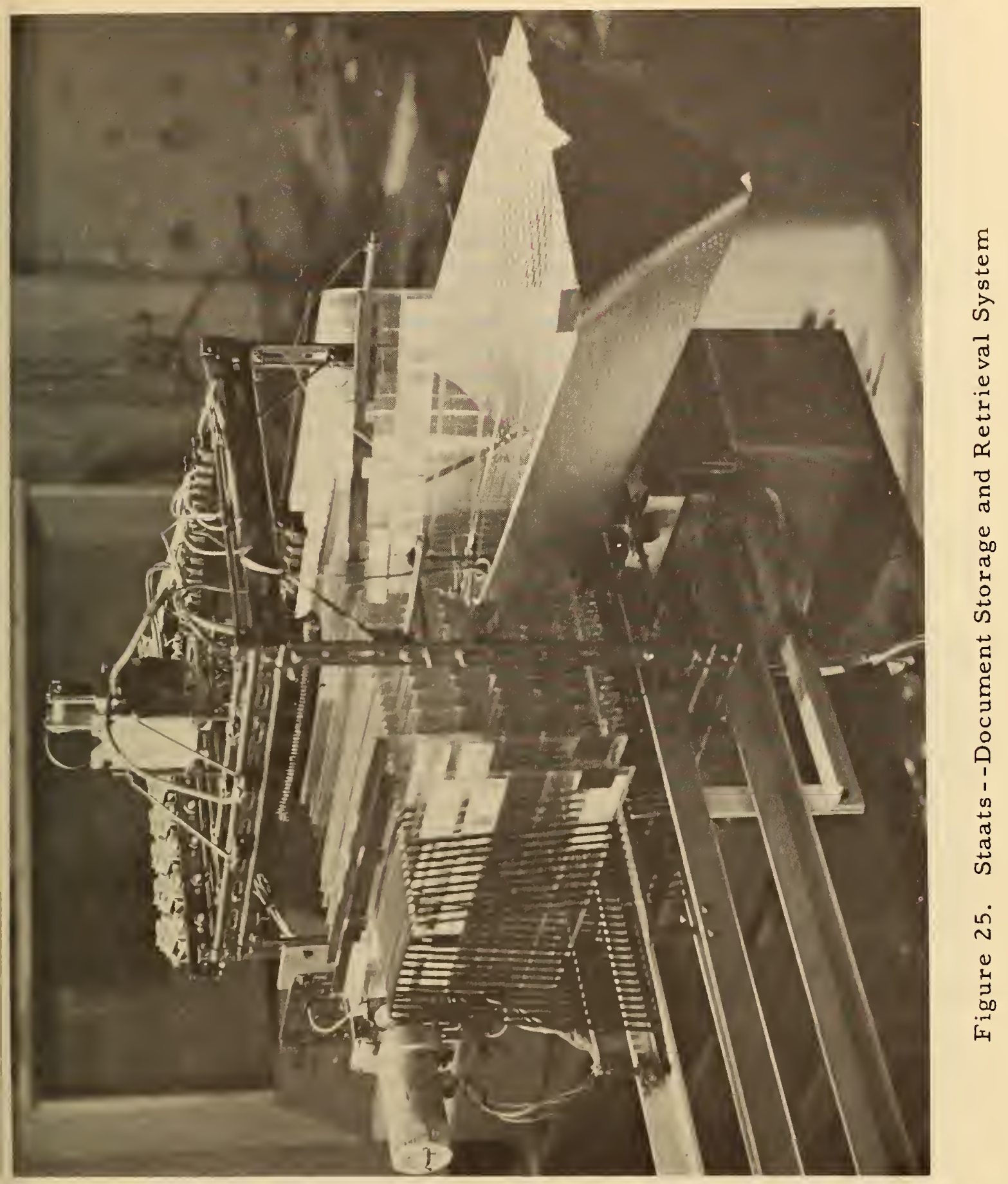


"Punched cards have also come into use in the filing of photographic negatives. E-Z file cards with Filmsort positive transparencies of the print have been used by Eastman Kodak for this purpose. Different classifications are necessary to suit the type of work being done by different photographic organizations. For the commercial photographer, the name of the subject or client serves as the best filing key. This is true also for such files used for police identification, and by industrial, personnel, passport and medical photographers. Commercial illustrators and industrial photographers, on the other hand, are more concerned with jobs or products and for them filing by product name, company or department, job name or job number is more desirable. Coded punching around the edge of the card carries the key to the filing classification for the photograph covered by each card and allows rapid mechanical selection of the desired cards. Print and data sheet transparencies may be examined directly from the card with a viewer without removing the negative from the file. This system fulfills the two most important requisities for filing negatives: prompt location and minimum handling to avoid scratching the surface and damage from dust particles. "I/

Mention was also made of the use of punched cards with microfilm inserts for the compact storage of blueprints and engineering drawings. These systems are of the address-type. The most commonly used method of retrieval is by manual extraction from the file. This method of record storage has found wide use in industrial and Government organizations, 2 including aboard ship. One example of an application may be found at the Wright-Patterson Air Force Base, where the Engineering Drawings Branch has over 6,000,000 aperture cards containing aircraft drawings. Another is at the Meat Inspection Branch of the U.S. Department of Agriculture, which has over 180,000 cards containing colored film inserts that illustrate meats, their markings, and their brands.

Another example discussed in the 1958 punched card survey was that of a market research application at the Magnaflux Corporation,

\section{1/ Ref. 50, p. 312 .}

2/ As early as 1955, military specifications for microfilming engineering drawings included a requirement for mounting the film in standard punched cards and punching into the cards selection information such as Federal Supply Code Classification and drawing number. Specification MIL-M18872 (Aer), June 13, 1955, is cited in Ref. 50, p. 338. 
which included a photographic illustration of the $5^{\prime \prime} \times 8^{\prime \prime}$ edge-notched cards containing microfilm copies of all correspondence with a particular customer. These were filmed on adhesive-backed film and mounted on the card. $1 /$

In addition, three specific examples were given of either microfilm insert or photographic card application to literature search problems. These were for (1) chemical literature, (2) reduced image versions of articles published in The Journal of the Society for Nondestructive Testing, $2 /$ and (3) geological data. 3 The chemical literafure instance follows the suggestions of $\mathrm{T}$. J. Williams and A. Rose. 4/ In this proposal, a standard 3" $\times 5^{\prime \prime}$ Microcard would be mounted on standard punched cards so that 20 columns would be left for a subject-matter classification code and 10 columns could be used for 4-digit random numbers for additional indexing and selection information.

The present state of the art with respect to the application of discrete record systems using microfilm inserted into aperture cards or mounted on other cards, which can be manipulated by sorting needles or by machines, continues to be marked by many storage applications that do not use mechanical selection. In most cases, it appears that difficulties in handling and machine manipulation of punched cards with these inserts preclude extensive use of searchtype selection methods. Also, when filing is by address, manual retrieval from a large address file is faster than any existing mechanical retrieval system. On the other hand, the carrier card provides ease of manual handling and protects the film insert itself. More significantly, the microfilm aperture cards lend themselves to appreciable economies of storage and effective use of machine manipulation techniques in initial sorting and file merging.

A representative example of a very large collection maintained in the form of microfilm inserts in IBM punched cards is the Intellofax system at the Central Intelligence Agency. The Intellofax System has been in use since 1953. It contains 2, 750, 000 aperture cards and grows at the rate of 250,000 cards per year. Searches are

1/ Ref. 50, p. 318. See also Cannon, W.A., Jr. "A punched card system for technical liaison, sales analysis, and file reductions," Ref. 47.

2/ Ibid, pp. 323, 339, and 319, respectively. See also p. 31 of this report.

3/ Ibid. See pp. 329 thru 333. See also Section 3.2. 1 of this report.

4/ Ibid, p. 323, citing Williams and Rose, "A solution to the problem of storage and availability of chemical literature",

Ref. 297. 
made manually on index and cross-reference cards which have a brief abstract of the document typed onto them. These cards list the document numbers that are used as addresses to enter a file of 16-mm type $E$ aperture cards. The aperture cards contain microimages of the original documents. Hard copies are made as they are required.

Two difficulties have been observed with this system. First, the use of a single card both for punched and for typed information limits the amount of either kind that can be recorded. The actual abstract, in particular, contains much less information than was available in the cover sheet prepared during analysis of the original document. Second, the time and cost involved in typing all of the cards required for cross-referencing and multiple-search-access purposes are high. For these reasons, a proposal by the Bell and Howell Company which would provide the means for a film chip replica retrieval system is currently being tried.

The Bell and Howell proposal originally suggested a copying machine (Document Abstract Retrieval Equipment, DARE) to produce on punched cards reduced size images of the full text of the cover sheets prepared by the document analysts. The techniques have been described, in part, as follows:

"In this system it is assumed that the analyst, when preparing the cover sheet for a document, has decided what indexing headings will be appropriate for that document in the master index file. The total number of headings is then indicated by a punched-hole code system at the bottom of the document cover sheet...

"Under the proposed system, the cover sheet.... will be placed in the feed hopper of the machine, and from then on processing will be automatic. As the machine takes the top cover sheet from the hopper and feeds it into the recording section, the number of duplicate IBM cards to be prepared from that cover sheet is sensed by the machine, and that particular cover sheet will be retained.... until the necessary number of image cards has been made....

"The image of the cover sheet, reduced approximately 3. 33X, would be focussed through a folded optical system onto a rotating selenium-coated Xerographic drum. This drum, which moves in synchronism with the document drum, is first sensitized by passing it under a corona charging station, then exposed in motion while it passes the exposure slit. The exposed area of the drum then rotates past the image-development section.... Following development, the image area rotates to the image-transfer station where an IBM card is fed against the powder image at the proper time. Electrostatic transfer will be used. The drum then rotates 
through a cleaning station where the residual powder image is removed, through a light-flooding station where the residual electrostatic image is removed, and then back to the charging station. The image-bearing card, meanwhile, passes through an image-fixing station and then into a cardreceiving hopper..." $1 /$

In practice, for the equipment now being tested, the reduction ratio is approximately $3: 1$ for cover sheets up to $81 / 2^{\prime \prime} \times 14^{\prime \prime}$ in size. It is anticipated that the input processing operations will proceed at the rate of 2,000 cards per hour when the machine is in operation. This rate accommodates 1,000 cover sheet recordings per day, assuming a 6-hour production period, and an average of 12 selectioncoded cards per document. The selection and retrieval operations in the DARE system will then proceed by conventional punched card processing means. These cards and their handling equipment possess characteristics similar to other systems described in this report. However, the cards are frequently retrieved manually by an alphabetical keyword address.

\section{5 Address-Type Selection Systems}

In addition to the systems and proposed devices previously described, a number of devices meeting the definitions of compressed storage, mechanical manipulation, and replica copy output have been and are being developed. In most cases, however, these additional systems incorporate integral indexing only to the limited extent of keys, marks, or codes corresponding to address-locations and are used only for address-type or file reference applications.

Table I (page 35) listed a number of these address-type machines. Most of them, as previously mentioned, will not be described in this report. However, there are several that appear to have the storage capacity and mechanical agility to be coupled as output devices with conventional computers. These computers could search a "deeply" indexed tape file and retrieve the desired document on the basis of an address location instruction to the storage device.

One of the earliest devices of this nature is the NBS micro-image locator $2 /$ developed in 1955. This uses a punched-paper-tape input as an instruction to print out selected frames of information. The retrieval time is less than two seconds per item for 10, 000 information items stored on a drum-mounted stabilized film sheet.

1/ "Proposal to Central Intelligence Agency for development of a device to prepare reduced-size images of analysts'cover sheets on IBM cards", Ref. 23.

2/ "An automatic micro-image file", Ref. 4. 
Another device, called Verac $903,1 /$ is being developed by the Avco Corporation. Laboratory models of the input camera, "direct access file" (selector), and the print-out components have been completed with some financial aid 2 / from the Council on Library Resources. This system uses a number of film or "memory" planes, each of which contains 10,000 document images. Address selection is accomplished by specifying the coordinates (column, row, and plane) of the item location. The camera is a precision step and repeat device. It photographs up to legal-size documents at either 70 to 1 or 140 to 1 reduction. Two film planes are made simultaneously, one for use and the other for a security file. The current rate of image recording is about four per minute. With automatic document handling equipment, Avco anticipates that this would increase to one document per second.

The storage medium is a high resolution, Lippman emulsion (EK $649 \mathrm{GH}$ ) coated on film strips. This emulsion has a resolution capability of 1,500 lines per $\mathrm{mm}$. A memory plane measures 7. 6" $\times 7.8^{\prime \prime}$ and is composed of film strips that may be individually replaced for updating purposes. At a reduction of 140 to $1,10,000$ images can be stored in ma ximum areas of 0.128 " $\mathrm{x} 0.192^{\prime \prime}$ for each image. The "direct access file" will store up to 100 image planes, or a total of 1,000,000 items, in a carriage. Upon instruction, the carriage moves the required image plane to the reading station. The plane is then slid from the carriage until the appropriate column is aligned with a reading head. While the plane is being positioned, this reading head moves vertically to the proper row level. Accurately placed index marks on the edges of the plane activate a monitoring servo system to precisely position the image. The output from a selected image can be either an optical enlargement transferred to $35-\mathrm{mm}$ microfilm or a full-size enlargement produced by video techniques. The latter is viewed on a cathode-ray tube or printed out from this by an electrostatic tube-printer (Print-a-Pix). The CRT viewing and/or recording may also be accomplished at remote points through utilization of commercial-type video signal transmission systems.

A third device, based upon the earlier Amfis system, $3 /$ has recently been developed by the Information Retrieval Corporation, 4 /

1/

"Technical investigation of elements of a mechanized library system", Ref. 32 .

2/ Council on Library Resources Third Annual Report, Ref. 58, p. 49 , "Information storage and retrieval equipment, "Ref. 7.

3/ Avakian, E.A. "AMFIS", Ref. 6.

4/ Information Retrieval Corp. "The command retrieval information system," Ref. 140 . 
a subsidiary of Information for Industry, Inc. The new system is called CRIS (Command Retrieval Information System). A prototype model of CRIS is expected to be completed in March 1962, to be followed by production models scheduled for delivery in June 1962 .

The storage medium used in the CRIS system is a film scroll of microfilmed images $17^{\prime \prime} \times 400^{\prime}$ containing slightly over 500,000 $81 / 2 " \times 11^{\prime \prime}$ page images. The reduction ratio for this packing density is 30 to 1 . Lesser reduction ratios or larger-sized items, such as drawings, may also be accommodated with a corresponding decrease in the number of items per scroll. Format may be either 11 35-mm frames per column across the film scroll, or these same 11 frames may be divided into nine subareas each. Selection and retrieval is by 7 -digit keyboard entry $1 /$ giving the address plus an indication of whether the whole frame or a subarea is desired. This permits the control of magnification for viewing as well as providing the necessary micropositioning. Average access time is approximately 20 seconds for a given item on a particular scroll. Scrolls are handled in cartridges which can be changed either manually or automatically. Output is by viewer-display with a microcopy print if desired. The price of the image locator and viewer will be approximately $\$ 26,000$.

There are a number of address-type systems which use film chips in one form or another. The Magnavox Company has developed the MEDIA 2/ system which uses a 16-mm film chip. Each chip has an image of two legal sized pages and a 17-digit code. This code is recorded in a human readable form for the manual in-filing and is separately encoded in a 4-bit binary form for machine search use. The chips are stored in groups of 200 in capsules. The retrieval machine searches through a manually loaded capsule on the two least significant code digits for the desired chips within that capsule. An enlarged hard copy of the selected item is produced by Xerographic techniques.

Houston-Fearless is building an "Automatic Image Retriever", which consists of a central control station and modular storage units. Each storage unit stores 5,120 cassettes containing photographic film chips $21 / 2^{\prime \prime} \times 31 / 2 "$ in size. Retrieval is accomplished by setting a keyboard or by using punched paper tape. Maximum retrieval time for any cassette is 7.7 seconds.

1/ At a later stage, it is envisioned that punched paper tape or computer control will be used for entry.

2/ The Magnavox Company, "MEDIA, Magnavox electronic dataimage apparatus", Ref. 168.

Tate, V.D. "Microreproduction", Ref. 270. 
In addition, there are film chip devices such as General Precision's "Telecard" system, which uses aperture cards and transmits the image by television; the Hughes Model D aperture card system; Ramo-Wooldridge's Metricard; 1 and U.S. Army Engineers' Target Map Coordinate Locator by Fairchild.

Systems employing continuous rolled film, or its equivalent, for address-type selection and retrieval include another Magnavox development, the Film Data-Recorder and Reader of 1956, 2 / and the FLIP (Film Library Instanteous Presentation) system developed by Benson-Lehner in 1957. 3/ More recent developments are represented by hardware, prototype models, or proposals such as the Lodestar (Recordak) with either Kodamatic or counter, 4 / Auto Tutor, $\underline{5}$ / the viewer reproducer model C \& E equipment (Hughes) $6 /$ and an MIT proposal in 1961 for a Reader-Finder. 77 Ferranti 87 has in use a number of Rapid Access Projectors which are similar to the Rapid Selector and the FileSearch, except that they use $16-\mathrm{mm}$ film in a continuous loop, have a limited code field (18 bits), and have only a direct viewing output. This equipment is used for locating price data at high speed (approximately one second per item). In addition, there are a number of "teaching machines" or aids available that use a simple address-type serial selection.

\section{SYSTEM DESIGN CONSIDERATIONS}

In the design of a specific system for information storage, selection, and retrieval, we have previously noted two major factors. These are the needs of the particular application and the means available for effective man-machine communication. There are, in many cases, many degrees of interaction between these factors and the

1/ Ref. 243 .

2/ Refs. 169 and 178 .

$3 / \operatorname{Refs} .26$ and 27.

4/ Refs. 116, 236, 237, 246 and 277.

5/ Ref. 63 .

6/ Refs. 132, 133 and 134 .

7/ Ref. 62 .

8/ Ref. 88 . 
characteristics of storage media, selection equipment, and output techniques that might be combined in a given system.

The results of human analysis, which are used to identify, index, or classify the items that are to be stored, comprise a set of one or more search entries, file access points, or selection criteria. Preparatory operations such as transcription from handwritten records to machine-usable language are usually required. These inscriptions then may be affixed as labels to the items they represent, or they may be recorded on separate carriers which in turn are filed or stored. For the devices considered in the present report, $1 /$ integral indexing is used, so that selection criteria are attached to or inscribed on the same storage medium as the item images.

The selection criteria may or may not be encoded before they are recorded. Whether or not they are encoded, the format and capacity of the space in the storage medium is a condition limiting certain aspects of system design. For example, the total space available for storing the coded data for each item may restrict the number of selection criteria that can be assigned per item. Limitations of this selection code space may also dictate the use of special coding schemes such as superimposed coding. Choice of inscription methods and storage media usually reflects observed and predicted requirements for future use--the relative permanence of the inscribed marks, erasability of the storage medium, and ease with which new information can be inserted--as well as considerations of cost, convenience, and availability.

Other system design considerations reflecting the importance of effective man-machine considerations occur with respect to the formulation of specific search prescriptions. The subsequent process of search will involve one or more operations of inspecting stored items (or, more precisely, the records of the selection criteria assigned to the items) to select those which match one or more specifications of desired characteristics which an item should have if it is to be retrieved. A prerequisite for the conduct of a search, consequently, is a precise statement of the requirements upon which the search would be based.

The search prescription is typically composed of statements of what the selection criteria are and of relationships that should be found to obtain between several such criteria. As appropriate, the search prescription may also include statements limiting the desired scope of the search or setting other restrictive conditions. Examples of possible search prescriptions are thus: "A, B, or C, and D, but not $E ; "$ "A, B, and $C$, if published after 1960"; and "A, B, and $C$

Microcite is an exception, see Section 3.3.1. 
except Russian language items." Here again we find that machine characteristics, such as the extent of logical manipulation permitted in the search process, will affect the choice of equipment for a particular system.

The primary considerations and the most critical problems have to do with the human user. The search prescription as proposed by the user may or may not reflect accurately what he needs or even what he wants. What he does ask for will be determined by one or more of the following factors:

1. Detailed conscious awareness of the specific characteris tics which items must have in order to be relevant to the query;

2. Understanding of the current state of knowledge in a particular field and in other fields that may be related;

3. Understanding of the terminology in current use in that field or fields;

4. Preconceived notions as to what is apt to be found;

5. Willingness to undertake additional work in further screening and selection from the items that may be delivered as the result of the search; and

6. Prior experience with the performance of the search system.

The search prescriptions thus reflect with varying precision a real or assumed user need. For most mechanized systems using currently available techniques, however, there are restrictions imposed by the machine. Terms used for the selection criteria and the grammar or format used to specify interrelationships between them must be suitable for machine processing. Often the search prescription must be framed in terms of a relatively limited and fixed vocabulary. The selection logic of a particular device may require a one-to-one correspondence between the terminology and format of the selection criteria comprising the search prescription and the terminology and format of the search-entry-record for a stored item.

The user himself may be required to frame his prescription in this way. On the other hand, special tools are often made available. These range from the provision of access guides or search notes that aid in defining the search terms used in the system to the services of a reference librarian or information specialist who 'negotiates' the search prescription in collaboration with the system customer. 
Machine techniques may ultimately serve as aids in these operations as well as in subsequent renegotiations of search prescriptions to improve the results of search, but such techniques are not as yet generally available. Machine techniques are available for some of the auxiliary operations of encoding and preparing the search prescription for automatic processing, including the possibility of translating a given natural language statement into the required machine language. It is to be noted that mechanized systems typically tend to restrict the ability of the user to alter the

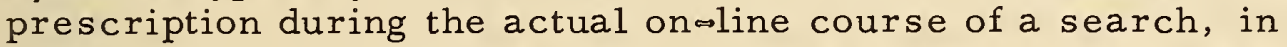
accordance with tentative results, and also tend to eliminate the possibility of "browsing". 1$]$

The actual conduct of search proceeds on the basis of the search prescription. Desired scope or limiting conditions are incorporated so far as possible into search strategy. That is, for example, if stored items are compartmentalized (e.g., in or on separate shelves, bins, drawers, tape reels, etc.) by language, date of issue, and the like, only those reels or bins which meet the limiting conditions are selected for actual processing. A number of different search prescriptions may be processed in parallel in some systems. If so, the conduct of actual searches may require scheduling, consolidation of several search prescriptions into a new master prescription, and provision for tagging the various selections so that they may be sorted out for the different customers. The usefulness of the output product will be determined not only by the relevance of the selected items to the user's question but also by the physical appearance and legibility of the items delivered to him and the ease with which he can use them directly.

The critical requirements in systems design fall into the areas of input preparation, input processing, file organization, search and selection, retrieval and output, and provision for making changes in the system such as updating, reclassification, and expansion. With respect to input preparation, we find the basic problems of item identification, item categorization, and other accessioning, cataloguing, and indexing requirements. The input processing operations necessary to store retrievable information items involve means for handling text and graphic information, and for recording selection criteria assigned. File organization involves not only storage, handling, and security considerations but also the question of the extent to which compartmentalization by subject matter is or is not feasible.

1/ Note, however, possibilities for free browsing due to preorganization of the file in the FileSearch and Microcite systems. 
In the subsequent search and selection requirements, there is first and foremost the crucial problem of adequate formulation of search prescriptions, together with questions of selection logic, search strategies, and possibilities for multiplexing or making many searches in parallel. Under typical output requirements, there are questions of the kind, quality, and relevance to the user of the products of the search; provision for rapid retrieval or on-line display; and compatibility with dissemination or communication systems. System considerations relate to changes in file organization, the required selection logic, and the possibilities for revising and updating the contents of either items or selection criteria records.

More specific considerations in the design of a particular information selection and retrieval system typically involve such problems as (1) storage economy, (2) effective capacity of the total system, (3) adequate selectivity in search, (4) speed of search and retrieval, and (5) compatibility of the output product with other operating requirements. For example, the limited storage space available for the necessary engineering drawings aboard a nuclearpowered submarine dictates a medium capable of greatly compressed storage; thus, microfilm aperture cards are now being used for this purpose. Output requirements may be for direct input to a facsimile transmission system $1 /$ or for copy of a quality likely to meet
customer acceptance, as in a sales or order fulfillment application. $2 /$

\section{THE STATE OF THE ART AND PROSPECTS FOR FURTHER DEVELOPMENTS}

System design considerations and comparative equipment characteristics meet together to provide a picture of the state of the art with respect to present or potential applications of document retrieval devices such as those discussed in this report. Some or all of the advantages of compressed storage, integral indexing, mechanical search, and facsimile replica output may suggest the choice of any one of these devices for use in a particular system.

1/

Ba!l, H.R. "Bureau of Ships High Speed Facsimile Printout", Ref. 16.

McMurray, J. P."The Bureau of Ships Rapid Selector System", Ref. 177.

2/ See, for example, Vander Wolk, L. J. "Teletype et Microfiche Transperente dans les relations entre bibliotheques", Ref. 287 which discusses possibilities for combinations of teletype communication with transparent microcards in connection with interlibrary loans and exchanges. 
The state of the art with respect to any given type of equipment for application in information selection and retrieval systems has at least three major aspects. The first, and most important, is the extent to which the system design meets real user needs and permits effective man-machine communications. The second involves the extent to which the characteristics of hardware, selection code capacity, programmable logic, and the like limit or constrain the system designer's choices in setting up a system intended to meet as effectively as possible the system objectives. The third factor is the extent to which systems have actually been used, so that the results of practical operating experience in one or more specific situations can be extrapolated to other possible applications.

We shall consider the present state of the art first, in terms of available storage media and equipment; second, with respect to applications; and third, with reference to implications for further developments. A summary table of the characteristics of devices considered is provided in Appendix B to this report.

\section{1 Storage Media and Equipment}

The microphotographic and related techniques for compressed data storage in the devices we have described provide for significant economics of storage, ranging up to $10^{6}$ coded pages stored in a space of approximately one cubic foot. Most of the devices considered presently use standard microfilm, either $16-\mathrm{mm}, 35-\mathrm{mm}$, or $70-\mathrm{mm}$, in continuous rolled film, film chip, or sheet or strip form. The medium used is related to quality of output, in that good tonal quality for closely packed graphic information is not achieved with standard microfilm, although the quality is adequate for most purposes. However, high-resolution film and other media possessing good tonal quality are available for use in most systems when required. A research study is being made, under a grant from the Council on Library Resources, to investigate high-reduction microphotography at resolution capabilities up to 10 times greater than that available with conventional microfilm. 1

The advantages of compressed data storage are obvious. The principal disadvantages associated with greatly reduced image storage include the following:

1. As storage becomes more compact, the requirements for mechanical selection and handling become more critical.

2. Quality of the original information may deteriorate in the viewing or reproduction process.

$1 /$

Council on Library Resources 5th Annual Report, Ref. 59, pp. 23-25, and Recent Developments No. 53, Ref。ól. 
3. Auxiliary display and viewing equipment or a separate printing process must be used in order to make the output product $1 /$ visible to the eye of the customer.

Charles Bishop, discussing the use of the microcard or microfiche as such, lists requirement-questions which a re equally applicable to automatic selection and retrieval systems using microforms, as follows:

"1. Is the microform material to be used mainly for storage and preservation of the originals or will the microfilm collection be constantly consulted and manipulated?

2. If the microform collection is being used continually, are the documents actually being read extensively or are they only used for verification of a few details?

3. Are the microforms being removed from the collection so they can be regrouped for some new purpose?

4. Must there be extensive coding on each microform so the collection can be sorted to drop out documents with specific attributes?

5. Is it necessary to be able to make microcopies of the documents in the collection? Can these copies be supplied quite flexibily or must large segments of the collection be duplicated, giving wanted and unwanted material together?" 2 /

The respective advantages of a continuous storage medium, serially searched, and of discrete unit record processing have been separately exploited in various different devices covered in the present survey. In general, the continuous roll or serial medium provides protection against loss, misplacement, or temporary absence of individual items from the file. It does require an item-byitem serial search, at least of each storage unit (such as a reel of tape or microfilm) or of the entire unit. Insertion of new items into an ordered arrangement, deletion, and change of item content usually

1/ I. e., the sheet film card.

2/ Bishop, C. "Problems in the production and utilization of microfiche", Ref. 28, pp. 53-54. 
requires an extensive series of cut-and-splice operations, increasing the problems of file maintenance. On the other hand, compartmentalized working files, with duplication of items meeting a specific grouping requirement, can be readily produced as required.

Conversely, the discrete unit record or film-chip approach emphasizes the importance of file compartmentalization to pinpoint-and thus to shorten -- the search, selection, and retrieval processes. Duplicates may easily be made at the time of input to provide multiple cross-reference copies. In addition, comparative ease of insertion, deletion, or revision of stored items is claimed. Care in handling, whether manual or mechanical, is an obvious operational requirement. An additional factor, at least for some applications, is that the integrity of the file is not necessarily maintained. Thus, individual chips of items may be physically withdrawn from the main file or from sections of working files for viewing or copying. The latter disadvantage may be eliminated by provision of an inviolate master or security file, but only at the expense of added costs and of multiple-search processing of both the working and the master files to assure integrity of search.

The film sheet, strip, and equivalent approaches attempt to minimize the disadvantages and to capitalize on the advantages of both the serial and the discrete unit storage media. In particular, they are highly adaptable to subject matter and other search-grouping compartmentalizations of the file. Thus, for example, 18,000 copies of abstracts are provided on a single film sheet for selective viewing in the Microcite system; 1,000, 000 images per plane are recorded in the Verac 903 input process; and up to 2,000,000 coded pages may be provided per WALNUT file module.

The question of file organization with respect to subject-matter compartmentalization may or may not be of particular importance. The importance of this factor will generally depend upon the size and diversity of the collection even more than upon the possibilities for multiple or parallel search, or upon differences in search processing time. The question of access is also important. There has been considerable confusion in the literature between such terms as "random access", "randomly organized files", and "rapid direct access".

First, it is noted that search-type selection systems all imply some type of random access. That is, the addresses of items which will meet the search prescription requirements are not usually known in advance. Therefore, items are randomly located in the file and will be selected during the search process with an access time that is conditioned by the number of items stored per search unit and the speed with which such units may be searched. This is random access, but the search process is total, systematic, and usually serial for serially-arranged storage media. 
Conversely, in address-type selection systems, the location of a desired item in file must always be known in advance. Access may be serial, as in an operation checking the address-code-key of each item in turn until the desired one is found. In addition, many socalled "random access" devices may be employed for this purpose. What this means is that rapid direct access is provided to any specific item, wherever it may be located in the file, provided that the location is known. Amfis, CRIS, Verac and the NBS Microimage Locator System are among the examples that have been cited.

Randomly organized files provide that stored items may be located anywhere in the file as opposed to situations in which items must be inserted, and replaced, in a specified order. There are obvious advantages in file maintenance operations if no ordered arrangement for re-insertion, addition, or deletion of items need be followed. Normally, the randomly organized file implies a searchtype selection and retrieval system. Either address-type or searchtype selection methods may be applied to ordered files in contrast to randomly organized files. Ordered files when used in systems employing continuous roll or other large-capacity serially arranged storage media typically require cut, splice, and recopy procedures in order to insert new items, to delete items, or to revise contents or arrangements. On the other hand, discrete unit record systems which require an ordered organization to permit efficient address type selection and retrieval also require extensive file maintenance operations.

Limitations on the number of selection criteria allowed per recorded item, per search prescription, and per search run of the file are evident with most of the document retrieval devices considered in this report. The information-storing capacity of selection code areas per retrievable item ranges from only those necessary to give the file address, for example, the Verac 903, up to 2,500 to 3,000 bits worth of selection code field capacity, as in Minicard and Magnavue. The choice of any of these devices may therefore require specially designed coding, such as the use of superimposed random coding, 19 to maintain adequate selectivity. On the other hand, these media generally provide equivalent selectivity in any system where the indexing and selection information can be accommodated on a single punched card.

This state-of-the-art survey of storage and retrieval equipment reveals unresolved difficulties in all reported systems, regardless of whether they use chip, roll, or sheet media. In the Filmorex System, for example, problems of film chip curl have been noted under operating conditions. These difficulties may be minimized by

1/ Mooers, C.N. "Putting probability to work in coding punched cards",Ref. 185, Ref. 50, pp. 340-356. 
maintaining an air-conditioned environment, by choice of a film medium with sufficient sturdiness and thickness, and by proper film processing. In continuous roll systems, such as the Rapid Selector, the problem of reliability of a high speed clutch is encountered if on-the-fly copying is to be used. On the other hand, when the film medium which is moving at high speed is brought to a sudden stop for copying, special problems of wear and damage to the film may arise.

Notwithstanding difficulties of selection code capacity and engineering problems that are still arising in prototype models, the present state of the art offers a variety of choices to the system designer. Equipment and installation prices range from under $\$ 10,000$ to over $\$ 1,000,000$ depending on the needs of a particular proposed application. Assuming that the critical aspect of selection criteria assignment(based primarily on human analysis and indexing of document content) has been resolved, a user may then take advantage of the selection code and selection logic capabilities available in most of the document retrieval devices considered in this report.

In addition, we note that the possibility of the use of several level systems clearly exists. For example, as an indexing and coding system becomes more elaborate, i.e., includes genericspecific relationships, role indicators, and interfixing, the selection logic tends to grow to at least those proportions that require a small general-purpose computer. In this respect, it is technically feasible today to use computer-type selection logic in direct conjunction with storage-retrieval equipment that provides graphic replica copies as output.

Further, it is possible that the use of document retrieval devices of this type may be so planned that simple or routine selections can be executed by a directly-attached electronic assembly. This should be quite modest in size and cost. More sophisticated selections, dependent upon more complicated logic-relationship discriminations, would require separate operations using a more complicated system. Output might be in the form of "locator" codes for direct automatic retrieval. Alternatively, the output might consist of abstracts from which the querist would make a further personal selection prior to full-text retrieval, much as in the previously described DARE and Microcite Systems.

Moreover, advantage may be taken of technological possibilities available today without requiring the design of a completely mechanized selection and retrieval system. This is true both for the address-type selection systems using various media and for the microfilm aperture cards. The modified punched card containing an inserted photographic image has, as pointed out earlier, been offered as an effective medium for storage and retrieval of graphic data such as photographs, drawings, and maps. There are many 
millions of these aperture cards in use today. Few of these collections currently are being searched by mechanical means. The probable reasons are as follows:

1. The small number of machine-usable characters available for selection code use limits the amount of information that can be used for search-type retrieval.

2. The items contained in the cards are usually describable by an identifying label or number and are filed sequentially by the label or number; under these circumstances, manual retrieval from organized files is as fast or faster than machine processing rates.

3. Manual retrieval operations result in less wear and damage to the photographic material inserted in the cards.

4. There are some continuing mechanical problems with respect to the satisfactory adhesion of the photographic items to the card stock, etc.

Despite the difficulties, just listed, aperture cards can serve and are serving a very useful and valuable role in the storage of engineering drawings and similar items.

Likewise, the Rapid Selector type equipment (roll-film) can be used for information retrieval in the same way as a conventional punched card system (viz., to select and retrieve a single-line title and citation, plus address-location clues). When so used, gains in the order of 40 to 1 are possible not only in compactness of storage but also in processing time.

\subsection{Applications}

The state of the art with respect to the actual use of information selection systems providing replica copies is marked primarily by technological potentialities which have not as yet been widely applied. With the exception of Minicard, Filmorex, Petroleum Research, FOSDIC, and the Bureau of Ships Rapid Selector, the document retrieval devices considered in this survey have seen little or no practical use in search prescription applications. Even in the case of the exceptions, experience to date has been too limited or specialized to provide for comparative evaluation of total systems. Generally lacking are case histories that would provide important information as to the interaction between technological capabilities and the latitude available to the user in choosing means to meet his requirements.

In the period 1957-1958, the National Science Foundation undertook a survey of "non-conventional" (including mechanized) systems for the selection and retrieval of scientific information. 1 -

I/ Ref. 197. 
Of 30 systems reported at that time, all except two involved mechanized or machinable selection techniques, including those which use manual needle sorting or edge-notched cards. Some degree of integral indexing was involved in seven cases, including two nonmachine systems which required manual search of indexed folders or jackets. Only one of the systems reported involved the use of microform storage. This was at the Callery Chemical Company, and involved essentially a two-level system for the literature on boron compounds. Originally, abstracts were prepared on McBee edge-notched cards. As the file grew, it was decided to convert to a mechanized system, described in part as follows:

"The IBM card used has a microfilm insert on which appears the abstract for the reference coded on the card. The original McBee files were photographed for this purpose. When an abstract is microfilmed now, a positive copy of it is made for each IBM card needed, and rolls of the negatives are kept on file in serial-number order. The number of boron compounds mentioned in an article or research idea determines the number of IBM cards to be prepared, one card for each compound....

"The code system emphasizes data on individual compounds-their properties, reactions, uses, etc. Compounds are classified by structure rather than by name. The cards contain either the coded original reference or number of report .... code and subclass designation; compound code number... and general subjects..." 1 y

As of January, 1958, trial searches were being made on a sample file of 400 cards.

In Septemter 1959, a second NSF study reported no further data on the Callery experiments and added only one further example of a system combining microform storage, integral indexing, and mechanical selection--that of the Filmorex System at the Centre National deRecherche Scientifique in Paris, France. $\frac{2}{\text { This }}$ system also was designed to retrieve abstracts, and an average of eight indexing terms has been used to index these abstracts. A supplement to the second NSF report adds the examples of an addresstype selection system for a microfilm aperture card collection of

1/ Ibid, pp. 6-7; see also Maginnity, P. M. and J.J. Allison, "Use of machine-sorted punched cards in literature searching", Ref. 166.

2/ Ref. 198. 
engineering drawings at Bell Telephone Laboratories and of the Petroleum Research Corporation Systems previously discussed in this report. $1 /$

Thus, with respect to the actual use of information selection systems producing replica copies as output, the present state of the art may be summarized as follows:

An effective means for total system evaluation is currently lacking. This is primarily because the relatively few systems of this type now in operation are, quite properly, geared to particular needs. No single collection, subjected to the same depth of analysis and indexing, has as yet been studied in terms of comparative or parallel operation using various possible techniques. With respect to intercomparisons between several different systems, information as to practical operating conditions, costs, and benefits is generally lacking due either to the experimental status of the installation, the short period of time during which it has been in operation, or the classified nature of the application. The potential advantages of technological developments now available have thus not been fully tested to date.

Nevertheless, certain potential advantages of the selection and retrieval devices combining microform storage, integral indexing, mechanized search, and graphic replica output can be stated in general terms. If large collections are considered, it is obvious that the larger the file becomes the more difficult it is to maintain multiple access by different users. Thus, the integrity or completeness of the files becomes increasingly important. By this, we mean that if several different users require the same material at approximately the same time, no one misses or overlooks pertinent material simply because it is not in the file at the time of search. This requirement is readily met by document retrieval devices where the master file is never broken and where copies, not originals, are is sued to the user.

The use of photographic images or other microform versions for document storage permits the inclusion in the collection of a large variety of information items such as full text, maps, drawings, aerial photographs, laboratory notebook pages and other hand-written records, charts, and the like. By virtue of the high resolution of microform storage media, the physical volume of the file is greatly reduced over that required by the original items. In addition, the replica copy output feature provides that the querist may retain selected items for personal use, that copies may be transmitted over facsimile communication links to users in remote locations, and that

Ref. 199. 
copies of rare and one-of-a-kind originals may be made readily available to depositories and other collections elsewhere.

With few exceptions, there have been almost no publicly available studies or evaluations of systems as used in specific applications. Users and suppliers have claimed important advantages of continuous or chip film replica retrieval systems. The following advantages have been claimed for chip systems in particular:

"..... Microfilm has been widely used for certain types of information handling and has been effective especially where the high information storage capacity could be used to advantage. Microfilm in continuous roll form, however, has certain limitations where information must be manipulated, rearranged, and sorted, or where information must be added to or removed from a file. Punched cards, on the other hand, have desirable characteristics for manipulating, rearranging, and sorting information in a system. They can be removed from or added to a file readily. However, the information storage capacity of punched cards is of a low order, and this is a disadvantage for many information-system requirements.

"The Kodak Minicard combines the desirable characteristics of microfilm and punched cards. The Minicard has the high storage capacity of microfilm, has the abilities for manipulation which are characteristic of the punched card, and handles digital and graphic information in a single record medium.

"..... The Minicard System not only can serve in the areas now occupied by punched cards and microfilm, ... but the system offers new capabilities in the region which has no satisfactory medium at present, that is, where there is a requirement for high record, activity and high quantity of information per record." $1 /$

Advantages claimed by users of the experimental Bureau of Ships Rapid Selector system have been reported as follows:

"1. High-speed multiple-access search. Minimum search speed is approximately 5, 000 images per minute. 2 /

$1 /$

Users of Filmorex at RADC have paraphrased these stated claims, substituting only the word "Filmorex" wherever "Minicards" appears in the original. See also Ref. 282, pp. 20-21.

2/ This rate is presumably based upon estimates of a search speed of 20 inches per second as presently planned for later models. 
A capability of approximately 40 accesses to each document image is available.

2. Simultaneous search, selection, and copying.

3. Search and selection as an integrated operation. No artificial address, such as a document number or accession number, is ever used or required as a transition between search and selection. The search is conducted directly in terms of bibliographic data and/or in terms of subject-matter.

4. An inviolate file. The films used in Rapid Selector searches never leave the room where the machine search is made." I/

A further advantage of this system application in the Navy is the compatibility of the photographic copy output with the high-speed facsimile transmission system developed for and used by the Bureau of Ships.

In general, however, the respective advantages of continuous roll film, chip or sheet film systems, as well as the overall advantages of storage, selection and replica retrieval offered by these devices, must be appraised in the light of specific user requirements and system design objectives. Thus, as L. B. Heilprin has said:

"We are constantly asking: Is it more efficient to store on roll film than on flat.film? What is the optimal length of a roll of microfilm for rapid access in searching its content? What is the optimal width of a roll of microfilm? Is it $70 \mathrm{~mm}$, $35 \mathrm{~mm}, 16 \mathrm{~mm}$, or some intermediate width such as 21 to 22 $\mathrm{mm}$ ? Is it better to use a single frame, such as an insert, or a short linear' 'microfiche' of several such frames? All such questions are based on efficiency in achieving a given objective. And, of course, no general answer can be given. We must know the specific objectives." 2

\section{3 Implications for Further Development}

The advantages mentioned above, plus other potential benefits of the document selection and retrieval devices that provide graphic facsimile copies as output indicate that further experimentation and

1/

2/

Ref. 177.

Heilprin, L. B. "Communication engineering approach to microforms," Ref. 122, p. 214. 
test for various practical applications should be encouraged. Comparative tests to determine operational processing times and costs for different storage media and equipment, given the same depth of analysis and the same user queries, should be undertaken. Practical demonstrations may go far to overcome user inertia, user dis satisfaction with the product, and lack of familiarity with what these systems can potentially do.

Systematic efforts to determine realistic overall usage requirements will go far to speed the exploitation not only of systems of this type but of other nonconventional documentation techniques and new equipment developments as well. In this process, a distinction should be made between possible answers to the question "What can we live with or make short-term gains by means of?" and potential answers to the question "What do we really need?"

Determining what is really needed is the first and crucial step for effective system design. As we have previously noted, the choices available to the potential user in relating equipment possibilities to his needs are most critical with respect to input preparation and organization of the files for subsequent search. It is here that the interplay between depth of subject content analysis, choice of coding system, capacity for selection code representation, and the ability to provide an open-ended system to permit updating and reclassification on the basis of usage is most important.

There are, in addition, certain prospects for further technological developments that are implicit in the present state of the art. These include increasing use of erasable and rewritable media for selection code recording and storage. 1/ Further development and use of high resolution media which would be capable of higher quality output may be required to increase user acceptance of the search product. Further research on micro-image viewing and display equipment is also indicated. 2 /mprovement of engineering design to increase ease of operation, to provide for checking and error correction, to expand (and in some cases open-end) selection code

1/ As in several previously discussed systems using magnetic media for selection code recording. Also see Section 3 of this report.

2/ Such as work at Battelle Memorial Institute under a grant from the Council on Library Resources, for the development of improved micro-image reading techniques, Ref. 59, p. 50 , and Ref. 60. 
field capacity, and to increase selection-logic flexibility is already under way. I Improved engineering design for systems embracing a hierarchy of mechanized selection and retrieval devices will also be required for the large-scale systems that must meet many different requirements and accommodate many searches involving varying degrees of search complexity.

Nevertheless, the fundamental problems remaining are those of communication, analysis, and relevance to needs and end-use. These are primarily matters of human intellectual endeavor and are the heart of the overall information selection and retrieval problem.

1/ Certain industrial organizations other than those who have announced systems as covered in this report are actively engaged in research and development efforts in these and related areas. A representative example is that of the Itek Corporation, where a current program objective has been described as follows:

'Itek Laboratories' current graphic information handling research and development activities are primarily concerned with identifying and selecting from a file information that a user needs rather than some broad subject in which he is interested.

"The principal novelty lies in the dissemination capability which will be provided by a new type of distribution system. Simple and flexible methods have been conceived for controlling the physical handling of graphic information in accordance either with routing instructions or with file organization data, or with the nature of the information content of a unit record.

"The new techniques for guidance, propulsion and route selection offer a promising and highly flexible approach to the problems of maintaining and operating a file of graphic unit records, particularly in situations in which information has to be selectively distributed from a central file to a large number of users with differing specialized requirements." W. S. Dempsey, private communication, dated December 13, 1961. 
This point, which is also the key to the present state of the art with respect to selection systems producing replica copies, was cogently formulated as follows in the First Annual Report of the Council on Library Resources:

"We know how to stimulate the production of books to provide information in better form than do existing books; how to create and organize additional indexing and other bibliographic services; how to develop guides to library resources so as to assure access to materials even though unavailable locally; how to assemble through micro-copying or publishing projects the resources for research now scattered through the libraries and archives of the world so that almost any library might supply in compact form the materials which would otherwise take lifetimes of travel and sifting to discover...

"..... (but) even if cures were limitlessly applied in terms of present knowledge, the result would probably only, be a further glutting of the channels of communications. "I/

\section{ACKNOWLEDGMENTS}

Support for this survey of information selection systems providing graphic replica copies has been provided primarily by Grant CLR - 147 from the Council on Library Resources to the National Bureau of Standards. This is in connection with a continuing program to establish and maintain the Research Information Center and Advisory Service on Information Processing, which has been jointly supported by the National Science Foundation, the National Bureau of Standards, and the Council on Library Resources. A major function of this Research Information Center is to provide, from time to time, state-of-the-art reports on topics of interest to research workers and others concerned with improved utilization of scientific information.

Grateful acknowledgment is made of the contributions of many individuals and organizations active in the area of either development or application of document retrieval devices covered in this report. Their cooperation and assistance has been invaluable.

Permission to reproduce various photographs of equipment, coded item formats, and related drawings is also gratefully acknowledged. Especially, acknowledgement is made to Mrs. Eileen Bowers of the Carnegie Institution of Washington for Figure 1. ; Julius Bosen of the U.S. Weather Bureau for Figures 7 and 8; 
FMA, Inc., for Figures 9 and 10; Mr. George Lear of Eastman Kodak for Figures 15 through 19; and Mr. Richard Furman of IBM for Figures 23 and 24. In particular, the permission of Dr. Vannevar Bush to examine the original literature on the Microfilm Rapid Selector and the access to the archival records of Science Service provided through the courtesy of Messrs. Watson Davis and Calvin N. Mooers are greatly appreciated.

With reference to the preparation of this report, grateful acknowledgment is made to F.C. Rose for editorial revisions and for the design and compilation of Appendix B and the index, and to Miss Josephine L. Walkowicz for the preparation, checking, and editing of the bibliography. Acknowledgment is also made of the able typing, clerical, and processing assistance of Mrs. Anna E. Upperman, Mrs. Helen B. Grantham, and Mrs. Anna K. Smilow. 


\section{APPENDIX A: BIBLIOGR APHY}

Sumnaries of current work in the field of document retrieval including pertinent devices are provided on a periodic basis in the National Science Foundation's Publications "Current Research and Development in Scientific Docunentation," and "iTon-conventional Technical Information Systens in Current Use."

Tramples of surveys which cover some of the same or similar devices will be found in reperence numbers 21, 24, 30, 31, 46, 52, $70,123,139,184,191,229,255,258,260,273,284$. 
1. "Automated Machine Trains Men," Electronics, 33:17 (April 22, 1960) 39.

2. "An Automatic Micro-image File," Computers and Automation, 5:11 (November 1956) 26-27, 50.

3. "An Automatic Micro-image File," Journal of the Franklin Institute, 262:5 (November 1956) 396-398.

4. "An Automatic Micro-image File," National Bureau of Standards Technical News Bulletin, 40:7 (July 1956) 89-90.

5. AVAKTAN, EMIK A., Yonkers, N. Y. "Stored Function Calculator," U. S. Patent 2,610,791; patented September 16, 1952; filed April 18, 1950. Class 235-61.9.

6. AVAKIAN, EMIK A. and EUGENE GARFIEID. "AMFIS - The Automatic Microfilm Infornation System," Special Libraries, 48:4 (April 1957) 145-148.

7. AVCO CORPORATION, Cincinnati, Ohio. "Information Storage and Retrieval Equipment: A Proposal to the National Library of Medicine," March 22, 1961, 14 p., File No. E-3.4.987-261.

8. AVCO CORPORATION, Cincinnati, Ohio. "VERAC 903; a System for Data Indexing, Searching, Retrieving and Reproducing," undated.

9. AZARI, FRIC, ALFRED E. GRAY, HFRMAN J. MALIN, and HAROLD B. THOMPSON, assignors to The Magnavox Company, Los Angeles, Calif. "Card Feeding Apparatus," U. S. Patent 2,947,538; patented August 2, 1960; filed February 24, 1958. Class 271-11.

10. AZARI, ERIC and ALFRED M. NELSON, assignors to The Magnavox Company, Los Angeles, Calif. "Card Processing Apparatus," U. S. Patent 2,954,226; patented september 27, 1960; filed May 5, 1958. Class $271-5$.

11. BADGLEY, ROBERT F., WILLIFM A. WILKEN, and CHESTER TIETIG. "Automatic Conparator for Records," U. S. Patent 2,580,270; patented Decenber 25, 1951; filed October 4, 1947.

12. BAGG, THOMAS C. "RCA's Proposal for a Document Retrieval Device," Washington, D. C., National Bureau of Standards, September 13, 1960. (Mernorandum to the File). 
13. BAGG, THOMAS C. "Status of Rapid Selector Development at NBS," presented at the American Documentation Institute Annual Meeting, Lehigh University, Bethleher, Pa., October 22-24, 1959.

14. BAGG, THOMAS C. and SIDNEY GREIINALD. "The Rapid Selector Development Program at the National Bureau of Standards," Vashington, D. C., National Bureau of Standards, November 1958. (Unpublished report).

15. BAGLEY, PHILIP R. and JAMES W. PERRY. "Applicability of Newer Electronic Techniques to Information Searching," presented at the Symposium on Machine Techniques for Information Selection, Massachusetts Institute of Technology, Cambridge, Mass., June 10-11, 1952. Based on Bagley's M.S. thesis, "Electronic Digital Machines for High-speed Information Searching," Department of Electrical Engineering preprint, May 31, 1952, 12 p.

16. BALL, HOFARD R. "Bureau of Ships High Speed Facsimile Printout," Washington, D. C., U. S. Navy Department, Bureau of Ships, undated, $3 \mathrm{p}$.

17. BALL, HOWARD R. "Bureau of Ships Rapid Selector," Bureau of Ships Journal, 10:11'(Novernber 1961) 6-7.

18. BALL, HOAARD R. "Information Retrieval Systern, Bureau of Ships," Navy Management Review, 4:I (January 1959) 7-8.

19. BALL, HOIARD R. "Infornation Retrieval in the Bureau of Ships," Bureau of Ships Journal, 8:1 (May 1959) 5-8.

20. BALL, HOWARD R. "A Photographic System for Data Retrieval and Presentation." Washington, D. C., U. S. Navy Department, Bureau of Ships CP 57-863. Presented at the American Institute of Electrical Engineers Summer General Meeting, June 24-28, 1957.

21. BEDFORD, GNINDOLYN M. "Review of Current Machine Systerns for Handling Inforination." Paris, Advisory Group for Aeronautical Research and Development, February 1956, 17 p., Report No. AGARD 46. ASTIA doc. no. AD-138 063 .

22. BELL \& HONELL COMPANY, Rochester Film Division, Rochester, N. Y. "BelI \& Howell Microfilms," undated, 22p. Descriptive brochure.

23. BELI \& HOMLLI COMPANY, Chicago, Ill. "Proposal to Central Intelligence Arency for Development of' a Device to Prepare Reducedsize Inages of Analysts' Cover Sheets on IBri Cards," undated, $14 \mathrm{p}$. 
24. BELLO, F. "How to Cope with Information," Fortune, 62:3 (September 1960) $162-167,180,182,187,189,192$.

25. BENDICK, MARC. "Equipment for Automated Teaching," Santa Monica, Calif., System Development Corporation, March 28, 1961, 19 p., SP-272.

26. BENSON-LEHNER CORPORATION. "The FLIP." Los Angeles, Calif., December 1, 1957. Descriptive brochure.

27. BEITSON-LFHIIR CORPORATION. "Introduction to the FLIP (Film Library Instantaneous Presentation)," American Documentation, 8:4 (October 1957) $330-331$.

28. BISHOP, CHARLES. "Problems in the Production and Utilization of Microfiche," American Documentation, 12:1 (January 1961) 53-55.

29. BOHNIRT, LFA M. "Machine Searching -- Fact and Fiction," presented at the 6th Meeting of the Interagency Records Adrinistration Conference, Washington, D. C., April 15, 1960.

30. BOURNE, CHARLES B. "Bibliography on the Mechanization of Information Retrieval." Menlo Park, Calif., Stanford Research Institute, 1958, 22 p.; Supplement I, February 1959, 25 p.; Supplement II, February 1960, 14 p.; Supplement III, February 1961, 27 p.

31. BOURNE, CHARLES P. "The Historical Development and Present Stateof-the-Art of Mechanized Information Retrieval Systems," American Documentation, 12:2 (April 1961) 108-110.

32. BOWKER, K., L. H. MARTIN, E. J. LUCAS, and C. PHANEUF. "Technical Investigation of Elements of a Mechanized Library System." Cincinnati, Ohio, AVCO Corporation, January 11, 1960, 110 p., Final Report No. EN -6680.

33. BROIDO, DANIEI. (Cockfosters, near Barnet, Fingland). "Control Apparatus for Sheet Feeding Devices," U. S. Patent 2,358,051; patented September 12, 1944; filed May 18, 1942. Class 209-111.

34. BRUSTMAN, JOSEPH A., assignor to Remington Rand, Inc., New York. "Code Selector," U. S. Patent 2,482,242; patented September 20, 1949; filed June 9, 1948. 
35. BRYCE, JAMES T., assignor to International Business Machines Corp., New York. "Film Data Selectins and Viewing Machine," U. S. Patent 2,323,372; patented July 6, 1943; Piled June 27, 1940.

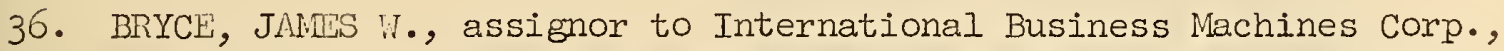
New York. "Photographic Statistical Machine," U. S. Patent 2,220,474; patented November 5, 1940; filed May 14, 1938. Class $95-4.5$.

37. BRYCE, JAIES N., assignor to International Business Machines Corp., New York. "Selective Film Printer," U. S. Patent 2,172,330; patented September 5, 1939; filed January 21, 1937. Class 83-24.

38. BRYCW, JMIES $H_{\bullet}$, assignor to International Business Machines Corp., New York. "Statistical Machine," U. S. Patent 2,124,906; patented July 26, 1938. Continuation of application Serial No. 64,639, February 19, 1936. This application June 4, 1938; Serial No. 211, 928 . Class $40-53$.

39. BRYCE, JAMES ., assignor to International Business Machines Corp., New York. "Statistical Machine," U. S. Patent 2,254,932; patented September 2, 1941; filed May 11, 1938.

40. BUCK, C. P., G. W. WALSH, and R. FORD PRAY, III. "Investigation and Study of Graphic-semantic Composing Techniques." Syracuse, T. Y., Syracuse University Research Institute, May 1961. College of Engineering, Report No. Eng. 695-615F, Contract AF 30(602)-2091; RADC TR 61-58. A.lso Final Report No. Eng. 695-615S1, June 1961.

41. BURKHARD, CHARLES A. "The Use of Punched-card Techniques in Preparing Reports, Papers, and Books." Chapter 11 in Punched Cards, Their Applications to Science and Industry, edited by Casey and Perry, New York, Reinhold Publishing Corp., 1951.

42. BURKIG, J. and L. I. JUSTICE. "Wagnacard - llagnetic Recording Studies," 1957 IRE IIISCON Convention Record, Vol. 1, Part 4, p. $214-217$.

43. BUSH, VANHWVAR. "As We May Mhink," Atlantic Monthly, 176:7 (July 1945) 101-103. Condensed and illustrated version appeared in Life, 19:11 (September 10, 1945) 112-114,116, 118,121,123,124. 
44. BUSH, VANNEVAR. Letter of April 15, 1940, commenting on the Howard-Wilson memorandum of April 1, 1940, Subject, Discussion of Possible Applications of the Rapid Selector; reply to Wilson letter of April 1, 1940. (Unpublished).

45. BUSH, VAINNEVAR. "Proposed Research on Rapid Selection." Unpublished memorandum later incorporated into Bush contract with the Massachusetts Institute of Technology, Research Corporation, National Cash Register, and Eastman Kodak, February 23, 1938. The contract was dated August 6, 1938.

46. BUSH, VANNEVAR. "Report to the Secretary of Commerce by the Advisory Committee on Application of Machines to Patent office Operations," Washington, D. C., U. S. Department of Commerce, December 22, 1954.

47. CANION, W. A., JR. "A Punched Card System for Technical Liaison, Sales Analysis, and File Reductions." Chicago, Ill., Hamilton Watch Co., Hathaway Instrument Division, undated.

48. CARLSON, C. O., D. A. GRAFTON, and A. S. TAUBAR. "The Photochromic Micro-image Memory," presented at the Symposium on Large Capacity Memory Techniques for Computing Systems, Washington, D. C., May 1961, sponsored by the Office of Naval Research.

49. CASEY, ROBERT S. and JAMES W. PERRY, editors. "Punched Cards, Their Applications to Science and Industry." New York, Reinhold Publishing Corp., 1951, 505 p.

50. CASEY, ROBERT S., JAMES W. PERRY, MADELINE M. BERRY, and ALLEN KINT, editors. "Punched Cards, Their Applications to Science and Industry." New York, Reinhold Publishing Corp., 1958, and edition, $697 \mathrm{p}$.

51. CHALKLEY, LYMAN. "Conditions for Projection Copying on Dye Cyanide Sensitized Materials," Photographic Science and Engineering, 3:4 (July-August 1959) $174-177$.

52. CHERENIN, V. P. and B. M. RAKOV. "Experimental Information Machine of the Institute of Scientific Information, Academy of Sciences, USSR" (Eksperimentalnaya Informatsionnaya Mashina Instituta Nauchnoy Informatsii Akademii SSSR), Mosrow, 1955, 40 p. 
53. CHRONIC, JOHT. "How Microfilm Library Aids Research," World Oil, 142 (May 1956) 95-97.

54. CIGANIK, MAREK. "Scientific, Technical, and Economic Information in a Research Organization," Proceedings of the International Conference on Scientific Information, Washington, D. C., November 16-21, 1958. Washington, National Academy of Sciences - National Research Council, 1959, Vol. 1, p. 613-647.

55. COILE, RUSSELL C. "University of Kentucky Libraries Occasional Contribution No. 6I," February 1954. Contains: "Libraries for Engineers and Scientists," and "Scientiric Aids to Docunentation."

56. "Convert Engineering Drawings to Microilim Mounted in Punched Cards," Industrial Laboratories, 9:8 (Ausust 1958) 40.

57. COUNCIL ON LIBRARY RESOURCES, INC., Washington, D. C. "Ist Annual Report for the Period Ending June 30, 1957."

58. COUNCIL ON LIBRARY RESOURCES, INC., Washington, D. C. "3rd Annual Report for the Period Ending June 30, 1959."

59. COUNCIL ON LIBRARY RESOURCES, INC., Washington, D. C. "5th Annual Report for the Period Ending June 30, 1961."

60. COUNCIL ON LIBRARY RESOURCES, INIC, Washington, D. C. "Battelle Given Contract to Seek Ways to Facilitate Reading of Microimages," Recent Developments No. 50 for release January 9, 1961.

61. COUNCIL ON LIBRARY RESOURCES, INC., Washington, D. C. "Investigation of Various Factors Affecting High-reduction Microphotography to be Made," Recent Developments No. 53 for release February 5, 1961.

62. COUNCIL ON LIBRARY RESOURCES, INC., Washington, D. C. "Grant Made for Construction of a Model for a Micro Finder-Reader System," Recent Developinents No. 65 for release October 15, 1961.

63. CRONDER, NORMNN $\Lambda$. "Automatic Tutoring by Intrinsic Programming." In: Teaching Machines and Programmed Learning, Washington, D. C., National Nducation Association, 1960, p. 286-293. 
64. DAVIS, HELEN M. "Proposal of Bibliographic Department of the Documentary Institute of Science Service," Washington, D. C., Documentation Institute of Science Service, Document No. 61, July 26, 1935. (Now in Archives of American Documentation Institute.)

65. DAVIS, WATSON. "Memorandum of Visit with Dr. Vannevar Bush, Dean of Engineering and Vice-President of Massachusetts Institute of Technology at Ann Arbor, November 15, 1932," Vashington, D. C., Documentation Institute of Science Service, Document No. Il, November 20, 1932. (Now in Archives of Aruerican Documentation Institute.)

66. DAVIS, WATSON. "Microphotographic Duplication in the Service of Science," Science, 83:2157 (May 1, 1936) 402-404.

67. DAVIS, WATSON. "Optical Problems Involved in a Comprehensive System of Bibliography," Journal of the Optical Society of America, 24:2 (February 1934) 58.

68. DAVIS, WATSON. "Remarks at the Banquet of the National Microfilm Association, Thursday, April 2, 1959, at the Mayflower Hotel, Washington, D. C." Proceedings of the Eighth Annual Meeting \& Convention Held in Washington, D. C., April 2-4, 1959. Annapolis, Md., The National Microfilm Association, 1959, p. 78-81.

69. DAVIS, WATSON. "Scientific Publication and Bibliography (Plans of the Documentation Institute of Science Service)," Washington, D. C., Documentation Institute of Science Service, Document No. 55, July 22, 1935. Presented before the 13th Conference of the Institute Internationale de Documentation, Copenhagen, Septerber 9-14, 1935. (Now in Archives of American Documentation Institute.)

70. DE GROLIER, ERIC. "International Advisory Committee for Documentation and Terminology in Pure and Applied Science," a preliminary report. Paris, UNESCO, August 24, 1955, 31 p. Ref. 320/5601; translated from the French.

71. DICKINSON, ARTHUR H. and ROBITRT I. ROTH, assignors to International Business Machines Corp., New York. "Data Reproducing Machine," U. S. Patent 2,224,764; patented December 10, 1940; Piled June 16, 1939. Class $164-115$.

72. DOCUMLITATION, INC. "The Minicard System. A Case Study in the Application of Storage and Retrieval Theory." Vashington, D. C., Technical Report No. 16, November 1956, 40 p., Contract Nonr-130500. ASTIA doc. no. AD-137 472. 
73. DOSS, MILJBURN PRICE. "Information Processing Equipment." New York, Reinhold Publishing Corp., 1955, 270 p.

74. DRAEGER, R. H. "A Proposed Photoelectric Selecting Mechanism for the Sorting of Bibliographic Abstract Entries from 35m. Film," Washington, D. C., Documentation Institute of Science Service, Document No. 62, July 27, 1935. (Now in Archives of American Documentation Institute.)

75. EASTMAN KODAK COMPAIY, Rochester, N. Y. Contract AF 30 (602)-1108. KUIPTRS, J. "Conference at Eastman Kodak Cormpany on the Minicard Project, July 1954." ASTIA doc. no. AD-76 265. TYLER, A. and W. MYIRS. "MINICARDS" - A Project Under Developrient at Eastman Kodak Company, May 1953 (revised March 1954). ASTIA doc. no. AD-76 266.

76. EASTMAN KODAK COIPANY, Rochester, N. Y. Contract AF 30(602)-1188, "The AFOIN-IB Minicard System (Minicard Information and Data Handling Systern - System Study Phase)."

Progress letter reports:

PLR-2, August 1954, PLR-3, September 1954, and PLR-4, october 1954, ASTIA doc. no. AD-55 082;

PLR-3, February 1955, ASTIA doc. no. AD-67 613;

PLR-9, March 1955, ASTIA doc. no. AD-67 612;

PLR-10, April 1955, ASTIA doc. no. AD-72 703;

PIR-11, May 1955, ASTIA doc. no. AD-72 704; PLR-13, July 1955, ASTIA doc. no. AD-81 639; PLR-14, August 1955, ASTIA doc. no. AD-81 640; PLR-15, Septeraber 1955, ASTIA doc. no. AD-90 063; PLR-16, October 1955, ASTIA doc. no. AD-100 199; PLR-25, October 1956, ASTIA doc. no. AD-114 209. 
77. EASTMAN KODAK COMPANY, Rochester, N. Y. Contract AF $30(602)-1698$; Project No. 4591, Task No. 45910; under sponsorship ôे Rome Air Development Center, ARDC, Griffis Air Force Base, N. Y.

Third Interim Letter Progress Report, Covering Period September 1, 1957-September 30, 1957. Report No. 1698-3, November 25, 1957; RADC-TN-58-39; ASTIA doc no. AD-148 584.

Interin Letter Progress Report, Covering Period October 1, 1957-October 31, 1957. Report No. 1698-4, December 12, 1957; RADC-TN-58-38; ASTIA doc. no. AD-148 582.

Interim Letter Progress Report Covering Period November I, 1957-Novernber 30, 1957, Vol. II. Report No. 1698-5, January 29, 1958; RADC-TN-58-89B; ASTIA doc. no. AD-148 661.

Interin Report Covering Period May 1, 1958-January 31, 1959, "Systems Study of Photographic Records and Services Division, Document Data Processing Central AN/GSQ-II(V)." Report No. 1698-SSI, July 27, 1959; ASTIA doc. no. AD-225 499.

"Specifications and Technical Descriptions of Minicard Handling Devices," Minicard Special Engineering Report 1698-I4, October 28, 1959; RADC-TN-59-361; ASTIA doc. no. AD-230 686.

"Minicard Code Reader Waveform Analysis," Minicard Special Engineering Report No. 1698-E6, October 30, 1959; RADC-TN-59-360; ASTIA doc. no. AD-230 685.

78. EASTMAN KODAK COMPANY. "Eastman Kodal Demonstrates High-speed Minicard System." Rochester, N. Y., November 17, 1958, 2 p. (News release.)

79. FASTMAIJ KODAK COMPANY. "The Kodak Digital Reader-Recorder, Model A." Rochester, N. Y., Final Report, June 1950, Contracts N6ori-205, NBonr-617.

80. EASTMAN KODAK COMPANY. "Microfilming with Kodagraph Micro-file Equipment and Materials." Rochester, N. Y., Kodak Industrial Data Book, 1952, $60 \mathrm{p}$.

81. EASTMAN KODAK COMPANY. "The Minicard Interim Development Program," U. S. Governnent Research Reports, 33:2 (February 12, 1960) 184. PB 142936.

82. FLESWORTH, R. S. "New Horizons with Microfilm," Anerican Docurnentation, 2:4 (October 1951) 221-228. 
83. ENGEL, LEONARD. "Science Overwhelms the Scientists," The New York Times Magazine, September 10, 1961, p. 82,84,86.

84. ENGINVERING RESEARCH ASSOCIATES, INC. Appendix I, "General Description and Proposed Technical Specifications for Microfilm Selector," to "Progress Report for the Microfilm Rapid selector." St. Paul, Minn. and Washington, D. C., November 24, 1947, Contract Cac-47-24.

85. ENGINERING RESEARCH ASSOCIATES, INC. "Report for the Hicrofilm Rapid Selector," St. Paul, Minn. and Arlington, Va., June 20, 1949, Contract Cac-47-24.

86. ENGSTROM, H. T. "Microfilm Selection Equipment in Information Work," Industrial and Engineering Chemistry, 42:8 (August 1950) $1460-1461$.

87. EVEN, ARTHUR D. "Engineering Data Processing System Design." Princeton, N. J., D. Van Nostrand Co., Inc., 1960, 282 p.

88. FTRRANTI-PACKARD ELECTRIC, ITD. "Rapid Access Look-up System," Toronto, Bulletin 460, undated, $4 \mathrm{p}$.

89. [File Search/, Communications of the ACM, 4:5 (May 1961) 245. (Paragraph from "News and Notices.")

90. FILMOREX. "The Filmorex System," Paris, undated, 2 p.

91. FILMSORT DIVISION, DEXTER FOLDER CO. "New Horizons with Microfilm." Pearl River, N. Y., undated, 35 p. Descriptive brochure.

92. FMA, INC. "FWA Filesearch." El Segundo, Calif., 1960. Descriptive brochure.

93. FMA, INC. "The FMA File Search System." El Segundo, Calif., January 4, 1960, 12 p. Descriptive brochure.

94. FNA, INC. "User's Guide File Search." El'Segundo, Calif., undated, $69 \mathrm{p}$.

95. FORTUNE, RAYMOIN L. "Document Processor Reads Coded Dots," Electronics, 29:12 (December 1956) 164-163. 
96. "FOSDIC -- A Filu Optical Sensing Device for Input to Computers," National Bureau of Standards Technical News Bulletin, 38:2 (February 1954) 24-27.

97. "FOSDIC II - Reads Nicrofilmed Punched Cards," National Bureau of Standards Technical News Bulletin, 4:1:5 (May 1957) 72-74.

98. "FOSDIC III to Assist in 1960 Census," National Bureau of Standards Technical News Bulletin, 43:6 (June 1959) 106-107.

99. "FOSDIC III, National Bureau of Standards, Washington, D. C.," Cormunications of the ACM, 2:7 (JuIy 1959) 59-60.

100. FRIEDMAN, VIIIIAM F. and VIRNON E. WHITMAN. "Electric Control System for Tabulating Cards, Documents, and the Like," U. S. Patent 2,224,646; patented December 10, 1940; filed April 22, 1937. Class 209-111.

101. GELB, JOHN. "Utilization of Magnacard as a Display Device." Torrance, Calif., Magnavox Research Labs., February 14, 1961 , 49 p. Final Engineering Report MRL No. R-357, Contract AF 30(602)2133, Project 4594; RADC TR 61-1. ASTIA doc. no. AD-25I 271 .

102. GENERAI ELECTRIC RESEARCH LABORATORY. "Thermoplastic Recording." Press Release and Press Conference, New York, January 12, 1960, published by Research Information Services, January 1960, 20 p.

103. GLENN, W. E. "New Color Projection System," Journal of the Optical Society of America, 48:11 (November 1958) 841-843. General Electric Research Iaboratory Reprint 3043.

104. GLFNN, W. E. "Thermoplastic Recording," Journal of Applied Physics, 30:12 (December 1959) 1870-1873. General Electric Research Laboratory Reprint 3350.

105. GOLDBERG, EMANUEI. "Statistical Machine," U. S. Patent 1,838,389; patented December 29, 1931; filed Apri1 5, 1928; filed in Germany April 12, 1927.

106. GOODALE, CHARLES J. "Accounting Machine," U. S. Patent 2,251,993; patented August 12, 1941; filed March 13, 1937. Class 234-1.5. 
107. GRAY, ALFRED E., HIRRARI J. MALIN, and HAROLD B. THOMPSON, assignors to Magnavox Company, Los Angeles, Calif., "Card Processing Apparatus," U. S. Patent 2,956,800; patented October 18, 1960; filed June 13, 1957. Class 271-5.

108. GRAY, ALFRED E., HERMAN J. MALIN, and HAROLD B. THOMPSON, assignors to Magnavox Company, Los Angeles, Calif. "Card Processing Apparatus," U. S. Patent 2,953,370; patented Septernber 20, 1960; filed April 13, 1959. Class 271-4.

109. GREANI $\Lambda$, EVON C., assignor to International Business Machines Corp., New York. "Data Sensing and Handling Apparatus," U. S. Patent 2,928,073; patented March 8, 1960; filed December 31, 1954. Class 340-149.

110. GREEN, JOHN C. "The Rapid Selector - an Automatic Library," The Military Engineer, 41:283 (September-October 1949) 350-352.

1Il. GREENOUGH, M. L. "New Uses of Microfilm with Electronic Scanners, a Progress Report on FOSDIC III." Proceedings of the 8th Annual Meeting \& Convention of the National Microfilm Association held in Washington, D. C., $\Lambda$ pril 2-4, 1959. Annapolis, Md., The National Microfilm Association, 1959, p. 279-286.

112. GREENOUGH, M. L. and E. C. PALASKY. "Functional Specifications for FOSDIC, Model PC-1." Washington, D. C., National Bureau of Standards, October 29, 1957, 68 p. (Unpublished report).

113. HAKSTEEN, BARBARA L. "Review of Applications." Chapter 14 in Punched Cards, Their Applications to Science and Industry, edited by Casey, Perry, et al., New York, Reinhold Publishing Corp., 1958, 2nd edition.

114. HAMOND, HILIAM. "IVolution of the ASTIA Nutonated Search and Retrieval System." Arlington, Va., Armed Services Technical Information Agency, January 1961, Iv. ASTIA doc. no. $\Lambda$ D-252 000.

115. HANDEL, PAUL W., assignor to General Electric Company, New York. "Statistical liachine," U. S. Patent 1,915,993; patented June 27, 1933; filed April 27, 1931.

116. H HRRIS, CHARLIS E., JR. "Microfilm Inage Retrieval Systems," presented to the Subcommittee on lilectronics of the National Association of Mutual Savings Banks, New York, March 22, 1962, $16 \mathrm{p}$. 
117. HAYES, R. M. "The Magnacard System," Preprint of a paper prepared for the International Conference for Standards on A Common Language for Machine Searching and Translation, September 6-12, 1959, Cleveland, Ohio, sponsored by Western Reserve University and the RAND Corporation.

-.-- -.-. Information Retrieval and Machine Translation, edited by Allen Kent. New York, Interscience Publishers, Inc., 1960, Chapter 19, Part 1.

118. HAYES, R. M. "Magnavox Activities in Data Processing." Chapter in Modern Trends in Documentation, Proceedings of a Symposium held at the University of Southern California, April 1958, edited by Martha Boaz. New York, Pergamon Press, 1959, p. 74-81.

119. HAYES, ROBERT M. and GEORGE W. MAYLE, assignors to Magnavox Company, Los Angeles, Calif. "Card Processing Apparatus," U. S. Patent 2,965,019; patented December 20, 1960; filed November 26, 1956. Class 101-53.

120. HAYES, ROBERT M., ALFRED M. NELSON, and JEROME B. WIENER, assignors to Magnavox Company, Los Angeles, Calif. "Card Processing System," U. S. Patent 2,965,291; patented December 20, 1960; filed March 12, 1957. Class 235-61.7.

121. HAYES, ROBERT M. and JEROME B. WIENER. "Magnacard -- a New Concept in Data Handling," 1957 IRE WLSCON Convention Record, Vol. 1, Part 4, p. 205-209.

122. HEILPRIN, L. B. "Communication Engineering Approach to Microforms," American Documentation, 12:3 (July 1961) 213-218. --.--- --.. Proceedings of the Tenth Annual Mieeting and Convention, Chicago, Ill., April 4-6, 1961. Annapolis, Md., The National Microfilm Association, 1961, p. 80-92.

123. HELLER, ELMER $N$. and CHARLES D. HOBBS. "A Survey of Information Retrieval Equipment." Santa Monica, Calif., System Development Corporation, December 15, 1961, 30 p. SP-642.

124. HERNER, SAUL. "The Relationship of Information-use Studies and the Design of Information Storage and Retrieval Systems." Washington, D. C., December 8, 1958, 25 p. Technical Note No. 1, Contract AF 30(602)-1857, Rome Air Development Center, Griffis Air Force Base, New York; RADC-TN-59-136. AsTIA doc. no. AD-213 781. 
125. HERRICK, C. E., JR. "The Sensitonetry of the Positive Diazotype Process," Journal of the Optical Society of America, $42: 12$

(December 1952) 904-910.

126. "How MID Handles Label Control," The National Provisioner, 143:7 (August 13 1960) 18-20,42.

127. "205,000 Meat Labels on File -- How U.S.D.A. Can Pick Out Your Product Labels in a Few Seconds' Time," The National Provisioner, 128:13 (March 28, 1953) 25-27.

128. HOWARD, J. H. "A Brief Progress Report on the Rapid Selection Research." Memorandum to A. F. Sulzer, April 4, 1939, 6 p. (Unpublished).

129. HOWARD, J. H. "Description of the Rapid Selector Camera." Memorandum to Carroll L. Wilson, March 13, 1940, 2 p. (Unpublished).

130. HOWARD, J. H. "Discussion of Fossible Applications of the Rapid Selector." Mernorandum to Carroll L. Wilson, April 1, 1940, Il p. (Unpublished).

131. HOWARD, J. H. and L. R. STEINHARDT. "General Description of the Rapid Selector and the Apparatus for Preparing the Index Film." Memorandum to Carroll L. Wilson, March 12, 1940, $8 \mathrm{p}$. (Unpublished).

132. HUGHES AIRCRAFT COMPANY. "Introducing...Central File System." El Segundo, Calif., undated.

133. HUGHES AIRCRAFT COMPANY. "Introducing... Model "C" Visual Unit." El Segundo, Calif., undated.

134. HUGHES AIRCRAFT COMPANY. "Introducing...Model ' $D^{\prime}$ Card Sorter." El Segundo, Calif., undated.

135. HUGHES AIRCRAFT COMPANY. "Introducing...Rotary Magazine Visual (Testing Aid)." El Segundo, Calif., undated.

136. HUGHES AIRCRAFT COMPANY. "Introducing...Viewer Reproducer Model ' $\mathrm{I}$ '." El Segundo, Calif., undated.

137. "IBM Demonstrates Walnut," Computing News, 9:14 (July 15, 1961) $201-9$ - 201-10. 
138. "IBM Information Retrieval System Holds 99 Million Items, Finds One in a Moment," The Wall Street Journal, July 12, 1961, p. 28.

139. "Information Retrieval," Chemical and Engineering News, Vol. 39 (July 17, 1961) 102-110,112 and (July 24, 1961) 90-96,98.

140. INFORMATION RITRIEVAL CORPORATION. "The Command Retrieval Information System." Washington, D. C., undated, $3 \mathrm{p}$.

141. ISAACSON, S. "Technical Information 'The Paper Curtain' Industry Faces the Information Challenge." Orlando, Pla., The Martin Company, undated, $6 \mathrm{p}$.

142. JANES, PETSR. "A Photo-magnetic System for Docurent and Information Retrieval," Anerican Documentation, 10:4 (October 1959) 286-295. Yorktown Heights, N. Y., IBM Research Center Report RC-66, March 1, 1958, $17 \mathrm{p}$.

143. JONES, RICHARD E. "Actifiln in Action," American Documentation, 12:3 (July 1961) 222-223.

144. JONKER, FRFDERICK. "Design Considerations of Information Storage and Retrieval Machines." Washington, D. C., Documentation, Inc., April 1958, $22 \mathrm{p}$. Report to Air Force Office of Scientific Research, Contract AF 49(638)-91. ASTIA doc. no. AD-154 273 .

145. KAY, RONALD H., assignor to International Business Machines Corp., New York. "Information Storage and Retrieval System," U. S. Patent 2,982,176; patented May 2, 1961; filed November 28 , 1958. Class 88-24.

146. KERR, KINGDON and EDWARD HALITSKY. "Information Storage and Readout System," U. S. Patent 2,922,894; patented January 26, 1960; riled November 27, 1956. Class 250-219.

147. KIERSKY, LORETTA J. "Bibliography on Reproduction of Documentary Information, 1955-60." Reprinted with permission from Special Libraries in Proceedings of the Tenth Annual Meeting and Convention, Chicago, IIl., April 4-6, 1961. Annapolis, Md., The National Microlilm Association, 1961, p. 117-134.

148. KORTE, THOMAS H., THOMAS C: MYERS, and JOHN W. BERRY. "Microrilm Aperture Card Systen." Wright-Patterson Air Force Base, Ohio, Directorate of Engineering Standards, final report [1959] Iv., WADC TR 59-746. ASTIA doc. no. AD-232 960. 
149. KREITHEN, ALIXANDER. "Mathenatical Foundations for a Storage and Retrieval Theory." Washington, D. C., Documentation, Inc., June 1957, 15 p. (part 1 of 3). Directorate of Advanced Studies, Air Force Office of Scientific Research, Contract AF 4.9(638)-91; AFOSR TN 57-400. ASTIA doc. no. AD-132 475.

150. KUDER, MILTON L., assignor to the U.S.A. as represented by the Secretary of Comerce. "Automatic Information System," U. S. Patent 2,907,011; patented Septernber 29, 1959; filed June 28, 1957. Class 340-174.

151. KUIPERS, J. W. "Wicrocards and Microfiln for a Central Reference File," Industrial and Engineering Chemistry, 42:8 (August 1950) $1463-1467$.

152. INUIPERS, JOHIN . "Needed Research for Machine Information Systems." Paris, Advisory Group for Aeronautical Research and Developient, North Atlantic Treaty Organization, February 1956; AGARD Report 47. ASTIA doc. no. AD-126 713.

153. KUIPERS, J. W. "A Prograrn for the Development of an Information Searching System," Automatic Documentation in Action (ADIA), Trankfurt/Nain, Internationale Arbeitstagung, June 9-12, 1959, Preprints, p. 15.

154. KUIPIRS, J. W., A. W. TYLIR, and W. L. MYERS. "A Minicard Systen for Documentary Information," American Documentation, 8:4 (October 1957) $246-268$.

155. LABORATORIES FOR APPLIED SCIENCES, U. OF CHICAGO, ILL. "Materials and Techniques for Information Storage." Report for February 5Decermber 31, 1960, Contract AF 33(616)6061, Project 6239, Report No. LAS-TR-P188-10, January 1961, 22 p.; WADD TR 60-925. ASTIA doc. no. $\Lambda \mathrm{D}-251219$.

156. LaFOND, CHARLES D. "Microfiln TV Vay Cut Duplication," Missiles and Rockets, 8:23 (June 5, 1961) 34,37.

157. LALLEY, J. M. "A Treasure Lost in Unread Wedges," The Washington Post, December 2, 1961, p. AlO.

158. LANDUYT, M. M. "Indexing Systems Suitable for Technical Literature Handling: a Bibliography," Special Libraries, 47:3 (March 1956) 124-125. 
159. LANGAN, JOHN F., by mesne assignnent to Film 'N File, Inc., New York. "Record Card," U. S. Patent 2,512,106; patented June 20, 1950; filed January 3, 1946 . Class 40-158.

160. LOCKE, W. J. "Microfilm Pushes Drawings Aside," Product Engineering, 29:44 (October 27, 1958) 44-47.

161. LOUGHREN, ARTHUR V., assignor to Hazeltine Research, Inc., Chicago, IIl. "Secrecy Communication System," U. S. Patent 2,402,058; patented June 11, 1946; filed June 25, 1941.

162. LOUGHRIDGE, MATTHEW H. and ROBERT STUART. "Selective Photographing System," U. S. Patent 2,209,342; patented July 30, 1940; filed October 1, 1937.

163. LUHN, H. P. "Review of Information Retrieval Methods." Yorktown Heights, N. Y., International Business Machines Research Center, October 1, 1958, 10 p., Research Report RC-59.

164. LUTHER, FREDERIC. "Microfilm, a History, 1839-1900." Barre, Mass., Barre Publishing Co., 1959, 195 p.

165. "Machine Sorts Information--Fast," Business Week, No. 1055 (November 19, 1949) 41-44.

166. MAGINNITY, PAUL M. and NANCY J. ALLISON. "Use of Machine-sorted Punched Cards in Literature Searching of Boron Chemistry," presented before the Division of Chemical Literature, American Chemical Society, Septernber 1957.

167. THE MAGNAVO COMPANY. "Magnacard Specifications." Ft. Wayne, Ind., April 1, 1960, $58 \mathrm{p}$.

168. THE MAGNAVOX COMPANY. "MEDIA, Magnavox Electronic Data Image Apparatus." Ft. Wayne, Ind., undated. Descriptive literature.

169. MAGNAVOX RESEARCH IABORATORIES. "Film Data Recording and Reading System." Los Angeles, Calif., August 23, 1956, 4 p. Brochure.

170. MAGNAVOX RESEARCH LABORATORIES. "Operational Characteristics of the Magnacard Data Processing System." Los Angeles, Calif., undated. 
171. MANLY, RON. "The Inadequacy of Varying the Depth of Indexing and Other 'Document Collection' Approaches to Information Retrieval for Researchers," American Documentation, 12:3 (July 1961) 204-205.

172. MARTIN, T. C. "Counting a Nation by Electricity," Electrical Engineer, 12:184 (November 11, 1891) 521-530.

173. MASSACHUSEITS INSTITUTE OF TECHIOLOGY. "IThe Rapid Selector." Cambridge, Mass., July 1940, 9 p.

174. MAUCHLY, JOHI W. "Current Research Efforts and Trends in Storage and Retrieval: in the United States." Symposium on Collection, Storage and Retrieval of Information, Information Processing, Proceedings of the International Conference on Information Processing, UNESCO, Paris, June 15-20, 1959, edited by S. de Picciotto. Paris, UNESCO; Munchen, R. Oldenbourg; London, Butterworths, 1960, p. 495.

175. McCALL, B. F. "The Minicard System Applied to Research Reports," presented at the American Documentation Institute Annual Meeting, Lehigh University, Bethlehem, Pa., October 22-24, 1959.

176. MCMAHON, GEORGE T. "The Use of the Filmsort Card in Industry; The American Documentation Institute Meeting in Miniature," American Docurnentation, 12:3 (July 1961) 219-222.

177. MCMURRAY, JAMES P. "The Bureau of Ships Rapid Selector Syster." Preprint of paper presented at the American Documentation Institute Convention, Boston, Mass, November 5-8, 1961.

178. McPHERSON, R. G. "Film Data-recording and Reading Equipment," IRE Transactions on Instrumentation, I-6:3 (September 1957) $192-194$.

179. "Microcite, an Aid to More Effective Referencing," National Bureau of Standards Technical News Bulletin, 41:9 (September 1957) $141-142$.

180. "Microfilm Rapid Selector," Chemical and Engineering News, 27:41 (October 10, 1949) 2911.

181. MIULIO, N. "Docurnent Data Processing Central AN/GSQ-IIA(V) (Minicard). Categories I and II Test Report." New York, Rome Air Development Center, Griffis Air Force Base, September 1961, 68 p., RADC-TR-61-137. 
182. MOOERS, CAIVIN N. "Application of Random Codes to the Gathering of Statistical Information." Boston, Zator Co., 1949, 28 p., Technical Bulletin No. 31.

183. MOOERS, CALVIN N. "Coding, Information Retrieval, and the Rapid Selector." Boston, Zator. Co., 1951, 10 p., Technical Bulletin No. 57.

184. MOOERS, CALVIN N. "The Next Twenty Years in Information Retrieval. Some Goals and Predictions." Cambridge, Mass., Zator Co., March 1959, 18 p., AFOSR TN-59-245. ASTIA doc. no. AD-212 225.

185. MOOERS, CAIVIN N. "Putting Probability to Work in Coding Punched Cards - Zatocoding." Boston, Zator Co., 1947, 9 p., Technical Bulletin No. 10 (out of print). Superseded by "Zatocoding for Punched Cards," Technical Bulletin No. 30, 1950, 19 p.

186. MOOERS, CALVIN N. "Use of Magnacard Machines in Large-scale Information Retrieval Systems." Cambridge, Mass., Zator Co., May 1, 1959, 26 p., V-124. Invited address at the Magnacard symposium, Santa Monica, Calif., November 0-7, 1958.

187. MOOERS, CALVIN N. "Zatocoding and Developrents in Information Retrieval." Boston, Zator Co., 1956, 20 p., Technical Bulletin No. 103. Paper presented to the Aeronautical Group, Association of Special Libraries and Information Bureaux, at the Royal Society of Arts, London, September 7, 1955.

-...- -.-.-. ASLIB Proceedings, 8:1 (February 1956) 3-22.

188. MOOERS, CALVIN N. "The Zator - A Proposal, A Machine for Complete Documentation." Boston, Zator Co., 1951., Technical Bulletin No. 65 .

189. "More Instant Literature," Chemical and Engineering News, 37:26 (June 29, 1959) 82-83.

190. MORSE, RICHARD S., assignor to Eastman Kodak Co., Rochester, N. Y. "Rapid Selector-Calculator," U. S. Patent 2,295,000; patented September 8, 1942; filed June 23, 1938.

191. MUELLER, MAX W. "An Evaluation of Information Retrieval Systems." Burbank, Calif., Lockheed Aircraft Corp., Septernber 30, 1959, 114 p., Memorandum Report No. MR-7170. 
192. MYFRS, T. L. and G. L. LOOMIS. "The Minicard Film Record as a Common-language Medium." Preprint of a paper prepared for the International Conference for Standards on A Comon Language for Machine Searching and Translation, September 6-12, 1959, Cleveland, Ohio, sponsored by Western Reserve University and The RAIND Corporation.

Translation, edited by Allen Kent. New York, Interscience Publishers, Inc., 1960, Chapter 20, Part 1.

193. NATIONAL BUREAU OF STANDARDS. "An Automatic Micro-image File." Washington, D. C., June 1956, 3 p., Summary Technical Report 2010.

194. NATIONAL BUREAU OF STANDARDS. "The Rapid Selector." Washington, D. C., July 1940, 9 p. (Unpublished).

195. NATIONAL BUREAU OF STAINDARDS. "The Rapid Selector -- An Automatic Document Retrieval Device." Washington, D. C., September 1959, 4 p., Summary Technical Report STR-2388.

196. "NBS' Rephrasing Searcher," Missiles and Rockets, July 10, 1961, p. 31 .

197. NATIONAL SCIENCE FOUNDATION, OFFICE OF SCIINTIFIC INFORMATION. "Non-conventional Technical Inforration Systems in Current Use." Washington, D. C., January 1958.

198. NATIONAL SCIENCE FOUNDATION, OFFICE OF SCIERCE INFORMATION SERVICE. "Non-conventional Technical Information Systems in Current Use, No. 2." Washington, D. C., U. S. Government Printing Office, September 1959. NSF-59-49.

199. NATIONAL SCIENCE FOUNDATION, OFFICE OF SCIENCE INFORLATION SERVICE. "Supplement to Non-conventional Technical Information Systems in Current Use, No. 2." Washington, D. C., U. S. Government Printing Office, 1960. NSF-60-14.

200. NISLSON, ALFRED M. "The Research and Development of the Magnacard System." Los Angeles, Calif., The Magnavox Company, September 1958, Iv., Final Engineering Report, Contract $\Lambda F$ 33(616)3370; WADC Technical Report No. 59-42]. ASTIA doc. no. AD-2].1 694.

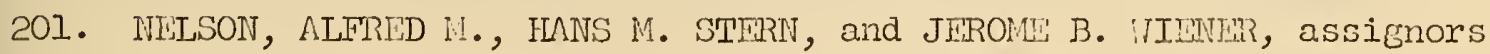
to Magnavox Company, Los lingeles, Calif. U. S. Patent 2,936,169; patented May 10, 1960; filed February 4, 1957. Class 271-5. 
202. NLLSON, A. M. , H. M. STERN, and L. R. WILSON. "Magnacard Mechanical Handling Techniques," 1957 IRE WESCON Convention Record, Vol. 1, Part 4, p. 210-213.

203. NELSON, CARL E. and HAROLD B. RUBIN. "Spectral Transmission of Microfilms as Related to Quality of Enlarged Electrostatic Prints." Proceedings of Eighth Annual Meeting \& Convention of the National Microfilm Association held in Washington, D. C., April 2-4, 1959. Annapolis, Ma., The National Microfilm Association, 1959, p. 127-154.

204. NNEH, FLOYD T. "The Kalfax System of Microrilm Duplication." Proceedings, Seventh Anmual Meeting, New Orleans, Louisiana, April 2l-23, 1958. Annapolis, Md., The National Microfilun Association, 1958, p. 27-40.

205. "New Desk-sized Information Retrieval System Can Handle Halfmillion Pages of Data," Business Week, September 2, 1961, p. 58.

206. "A New Microcite Machine for Large Scale Information Searching," National Bureau of Standards Technical Nevs Bulletin, 45:7 (July 1961) $113-115$.

207. NIESET, ROBERT T. "The Basis of the Kalvar System of Photography." Proceedings of Tenth Annual Meeting and Convention, Chicago, Ill., April 4-6, 1961. Annapolis, Wd., The National Microfilm Association, 1961, p. 177-191.

208. "Optical Punch Card - Electronic Brain Searches Literature With Combination of Microfilm and Punched-card Techniques," Chemistry and Industry, 65 (August 1949) 189-190.

209. ORDUNG, PHILIP F. and THOMAS C. BAGG. "Status Report on the Rapid Selector," Presented at The AIEE Surmer General Meeting, Montreal, quebec, Canada, June 24-28, 1957. Preprint CP 57-862.

210. PARKER, F. M. "Engineering Drawing Processing System," Special Libraries, 51:8 (October 1960) 4.29-432. Abstract in: American Documentation, 12:1 (January 1961) 70.

21l. PATTON, A. R. "Punch Card Filing System for Your Slides," The Camera, 73:1 (January 1950) 63,130. 
212. PERRY, JAMES $W$. and ROBERT S. CASEY. "Mechanized Searching." In: Encyclopedia of Chemical Technology, edited by Raymond E. Kirk and Donald F. Othmer. New York, The Interscience Encyclopedia, Inc., 1952, p. 449-467.

213. PERRY, J. W. "Photographic Selectors, the 'Rapid Selector' and Related Devices." In: Manual on Document Reproduction and Selection, The Hague, Internationa] Federation for Documentation, March 1957, p. 752El-752Ll, (April 1956).

214. PERRY, JAMES H., ALLEN KENT, and MADELINE M. BERRY. "Machine Literature Searching." New York, Interscience Publishers, Inc., $1956,162 \mathrm{p}$.

215. PETROLEUM RESEARCH CORPORATION. "How to Use the Micro-Research System; Sedimentary and Petroleum Geology Library." Denver, Colo., Instruction Booklet, revised september 1957, $41 \mathrm{p}$.

216. PETROLEUM RESEARCH CORPORATION. "Introducing an Entirely New Concept of Professional Libraries." Denver, Colo., Rocky Mountain Edition, undated, $12 \mathrm{p}$.

217. PETROLEUM RESEARCH CORPORATION. "Introducing an Entirely New Professional Library: The Micro Research System Medical Library on INeurology." Denver, Colo., undated, $14 \mathrm{p}$.

218. PETROLEUM RESIEARCH CORPORATION. "Introducing the Micro-Research System Vell Data Card File." Denver, Colo., Rocky Mountain Edition, undated, $18 \mathrm{p}$.

219. "Photo-electric Cells Sift Microfilm Records," Product Engineering, 20 (September 1949) 104-105.

220. "Photo-electric Librarian," Electronics, 22:9 (September 1949) 122.

221. "Photoelectric Search System Spots Digit Compatibility," Electrical Design News, 16 (October 1956) 16.

222. "The Pioneer Medals," Fourth Citation [to Vatson Davis]. Proceedings of Eighth Annual Meeting \& Convention Held in Washington, D. C., April 2-4, 1959. Annapolis, Md., The National Microfilm Association, 1959, p. 76-77. 
223. POFTER, R. W. "A Large-capacity Document Storage and Retrieval System." San Jose, Calif., Advanced Systems Developnent Division Laboratory, International Business Machines Corporation, April 14, 1961, 17 p., Technical Memorandum ASDJ-M-045.

----- -.-. Presented at the Symposium on Large Capacity Memory Techniques for Computing Systerns, Washington, D. C., May 1961, sponsored by the office of Naval Research.

224. POTIS, LOUIS M., assignor to Teletype Corporation, Chicago, Ill. "Tape Feed Alarm," U. S. Patent 2,351,229; patented June 13, 1944; filed April 27, 1942. Class 178-42.

225. POYLO, MICHAEI C. and ROBERT L. WHITTLE, assignors to International Telephone and Telegraph Corporation, Nutley, N. J. "Data Processing System," U. S. Patent 2,947,978; patented August 2, 1960; filed November 9, 1956. Class 340-174.

226. "Progress on the Rapid Selector and Other Shaviana," American Documentation, 2:2 (April 1951) 74 .

227. "Punch Cards Facilitate Rapid Searching for Spectrograms," Chemical and Engineering News, 30:49 (December 8, 1952) 5160-5161.

228. RABINOH, JACOB, assignor to the U.S.A. as represented by the Secretary of Commerce. "Optical Coincidence Devices," U. S. Patent 2,795,705; patented June 11, 1957; filed August 26, 1952. Class 250-219.

229. RABINOW, JACOB. "Presently Available Tools for Information Retrieval," Presented at the AIEE Summer General Meeting, Montreal, Canada, June 24-28, 1957. CP 57-860. -.--- --.-. Electrical Engineering, 77:6 (June 1958) 494-498.

230. RABINOW, JACOB. "Rapid Selector and Optical Dise Memories as Applied to Document Search," Presented at American Documentation Institute Annual Meeting, New York, December 1956.

231. RADIO CORPORATION OF AMERICA. "RCA Video File System," descriptive brochure, undated.

232. RADIO CORPORATION OF AMERICA. "Video File." Van Nuys, Calif., Brochure TP 1084, undated. Includes circulars describing system components: recorder, camera, printer, merge control, status and switching panel, monitor, soft copy reader, and master clock. 
233. "Rapid Selector," Chemical and Engineering News, 27:27 (July 4, 1949) 1955.

234. "The Rapid Selector, an Automatic Document Retrieval Device," Nationál Bureau of Standards Technical News Bulletin, 43:10 (October 1959) 178-179.

235. "The Rapid Selector, an Automatic Document Retrieval Device," Journal of Machine Accounting, 11:3 (March 1960) 38.

236. RECORDAK CORPORATION. "How to Index Your Microfilm Records...for Maximum Retrieval Efficiency." New York, descriptive brochure, undated.

237. RECORDAK CORPORATION. "How to Use the Recordak Lodestar Reader Model PS." New York, descriptive brochure, undated, $10 \mathrm{p}$.

238. RECORDAK CORPORATION. "Minicard Data Processing System." New York, Equipment Description Sheets, January 1960 and May 1960.

239. RECORDAK CORPORATION. "Recordak Automatic Image Retrieval System." New York, undated, 9 p. (Now out of print).

240. RECORDAK CORPORATION. "Recordak Films for Microfilming." New York, undated, 15 p., Parnphlet No. P-105.

241. RECORDAK CORPORAIION. "The Use of Aperture Cards for the Consolidation of Spectrophotometric Data." New York, undated, 7 p.

242. REX, FREDERICK J., JR. "Herman Hollerith, the First 'Statistical Engineer'," Computers and Automation, 10:8 (August, 1961) 10-13.

243. RICHOND, A. W. "Image Format Considerations in Photographic Intelligence Processing. Metricard Design." Canoga Park, Calif., Thompson Ramo Vooldridge, Inc., April 12, 1961, 55 p., DSPO Technical Note No. 14, Contract AF 30(602)1814; RADC TN 61-63. ASTIA doc. no. AD-258 149.

244. RIDFNOUR, L. M., et al. "Bibliography in an Age of Science." Urbana, University of Illinois Press, 1951.

245. RIDIR, FRIMONT. "Wicrocards, a New Form of Publication," Industrial and Engineering Chemistry, 42:8 (August 1950) $1462-1463$. 
246. ROSSE, EDIARD. "Microfilm as a Clerical Tool," reprint from The Office, October 1959.

247. RUBIN, H. E. "Sensitometry of New Diazo Films as Related to Specific Microfilm Applications." Proceedings of Tenth Annual Meeting and Convention, Chicago, IIl., April 4-6, 1961. Annapolis, Md., The National Microfilm Association, 1961, p. $202-225$.

248. SAMAIN, JACQUES. "Centre National de Recherche Scientifique, Paris, France." Non-conventional Technical Information Systems in Current Use No. 2, Washington, D. C., National Science Foundation, September 1959, p. 8-9. NSF-59-49.

249. SAMAIN, JACQUES. "Filmorex," brochure (in French), 1952, 11 p.

250. SAMAIN, JACQUES. "FILMOREX -- Eine Neue Technik Elektronischer Recherche für Information und Dokumentation," Nachrichten für Dokumentation, 9:1 (January 1958) 35-40. (In German).

251. SAMAIN, JACQUES. "The Filmorex System," Automatic Documentation in Action (ADIA), Frankfurt/Main, Internationale Arbeitstagung, June 9-12, 1959, Preprints, p. 32-34.

252. SCHUSTER, DONALD H. "'Mechanize'. Your Circuit Searches," Electronic Design, 9:16 (August 2, 1961) 214-216.

253. SEIDEIL, ATHERTON. "Reforms in Chemical Publication (Documentation)," Science, 80:2064 (July 20, 1934) 70-72.

254. SEIDELL, ATHFRTON. "The Photomicrographic Reproduction of Documents," Science, 80:2069 (August 24, 1934) 184-185.

255. SHAPIN, THEODORE, JR. "Automatic Searching System," U. S. Patent 2,923,921; patented February 2, 1960; filed June 23, 1954.

256. SHARP, HAROLD S. "Information Retrieval -- Pitfalls of Information Retrieval," Industrial Research, 3:2 (April-May 1961) 33.

257. SHAW, RALPH R. "High Speed Intermittent Camera," American Documentation, 1:4 (October 1950) 194-196. 
258. SHAit, RALPH R. "Machines and the Bibliographical Problems of the Twentieth Century." In: Bibliography in an hge of Science, Urbana, University of Illinois Press, 1951, p. 37-71. -..- -...... Reprint, "Bibliographic Organization." University of Chicago Press, 1951.

259. SHAW, RALPH R. "Management, Machines and the Bibliographic Problems of the Twentieth Century." Washington, D. C., U. S. Department of Agriculture, reprinted from "Bibliographic Organization," University of Chicago Press, 1951.

260. Shaw, RALPH R. "Nechanical Storage, Handling, Retrieval and Supply of Information," Libri, 8:1 (1958) 1-48. -..- -...... Report presented at the 6th meeting of the Documentation Cormittee, Advisory Group for Aeronautical Research and Development, February 20-25, 1956, in Rome. $\Lambda$ GARD Report 50, 1956. ASTIA doc. no. AD-144 216.

261. SHAN, RnIPH R. "Rapid Selector," Journal of Documentation, 5:3 (Decernber 1949) 164-171.

262. SHAW, RALPH R. "The Rapid Selector," The A.I.B.S. Bulletin, 4:3 (JuIy 1954) 20-21.

263. SHAW, RALPH R., assignor to the U.S.A. as represented by the Secretary of igriculture. "Systern and Apparatus for Selective Photographing," U. S. Patent 2,594,358; patented April 29, 1952; filed April 24, 1950.

264. SPRING, ELIZABETH. "S.L.A. New York Chapter Looks at Mechanical Aids," Library Journal, 75:8 (April 15, 1950) 722-724.

265. STAATS, HWIIY N. "Data Txtraction in Nondestructive Testing," Nondestructive Testing, 15:1 (January-February 1957) 44-46.

266. STAAMS, HFNRY N. "Description of the Staats Documentary Storage and Retrieval System," undated, 6 p.; addendum, 2 p., with Appendices $A-F$.

267. STERIV, JOSHUA, REYNOLD GREIRISTONE, and J. HOMARD WRIGHT. "Data Processing Devices and Systems." Washington, D. C., National Bureau of Standards, September 1955, 26 p. (Unpublished report.)

268. SULLIVAN, WALTRR. "Electronic Microfilrn Archive is Seen as an Aid for Overcrowded Libraries," New York Times, August 9, 1959, p. E9. 
269. TATE, VIRNON D. "An Ingenious Searcher," Section from "PMI In-Plant Reproduction," PMI - Photo Methods for Industry, 4:7 (July 1961) 66́-67.

270. TATE, VERNON D. "Microreproduction," PMI - Photo Methods for Industry, 4:9 (September 1961) 35,99,104.

271. TATE, VERINON D. "Photography in Research--Postwar," Review of Documentation, (Federation Internationale de Documentation, The Hague) 14:1 (1947) 8-14.

272. TATE, VERNON D., Editor. Proceedings, Seventh Annual Meeting, New Orleans, Louisiana, April 21-23, 1958. Annapolis, Md., The National Microfilm Association, 1958, $209 \mathrm{p}$ :

---- ---.--.. Proceedings of Eighth Annual Meeting \& Convention of the National Microfilm Association held in Washington, D. C., April 2-4, 1959. Annapolis, Md., The National Microfilm Association, 1959, $298 \mathrm{p}$.

-.-- --.--.. Proceedings of the Ninth Annual Meeting and Convention held at New York, April, 1960. Annapolis, Mid., The National Microfilm Association, 1960, $244 \mathrm{p}$.

-.- -..-... Proceedings of Tenth Annual Meeting and Convention, Chicago, II]., April 4-6, 1961. Annapolis, Md., The National Microfilm Association, 1961, $305 \mathrm{p}$.

273. TAUBE, MORTIVER and HAROLD NOOSTER, Editors. "Information Storage and Retrieval, Theory, Systems, and Devices." New York, Colurnbia University Press, 1958, 228 p.

274. TAUBE, MORTIMER. "New Tools for the Control and Use of Research Materials," Proceedings American Philosophical Society, 93:3 (June 1949) 248-252.

275. TAUBER, MAURICE F. "Problems in the Use of Microfilms, Microprint, and Microcards in Research Libraries," Industrial and Engineering Chemistry, 42:8 (August 1950) 1467-1468.

276. TAUSCHEK, GUSTAV. "Reading Machine," U. S. Patent 2,026,329; patented December 31, 1935; filed May 27, 1929. Class 250-41.5.

277. THOMAS, A. J. "Rapid Roll Film Image Retrieval Systens." New York, Recordak Corporation, undated, $3 \mathrm{p}$. 
278. THORENSON, R. "The Magnacard Development," presented at the Surmer General Meeting, American Institute of slectrical Engineers, Montreal, quebec, June 24-28, 1957. Preprint CP 57-861.

279. TOMISEITD, LEONARD G. "Apparatus for Making and/or Utilizing Photo Records," U. S. Patent 2,140,567; patented December 20, 1930; filed February 8, 1937. Class 88-24.

280. TONISEIND, IEONARD G. "Control Device for Indexing and Phototranscription Machines," U. S. Patent 2,215,381; patented September 17, 1940; filed August 10, 1939. Class 40-53.

281. TOMNEND, LEONARD G. "Method of and Apparatus for the Indexing and Photo-transcription of Records," U. S. Patent 2,121,061; patented June 21, 1938; iiled July 3, 1936. Class 40-53.

262. TYLER, A. H. , W. I. MYERS, and J. W. KUIPERS. "The Application of the Kodak Hinicard System to Problems of Documentation," American Docunentation, 6:1 (January 1955) 18-30. Presented at the Annual leeting, American Documentation Institute, Cleveland, November 4-5, 1954.

283. U. S. AIR FORCE. "The USAF Logistics Program for Engineering Data." Ohio, Wright-Patterson Air Force Base, Cataloging and Standardization Division, Directorate of Supply, Hq. Air Materiel Comrand, Septeinber 1, 1960, Publications SI-5, SI-5-1, and $S-1-5-2$.

284. U. S. COINGRESS. "A Study of Federal and Non-Federal Science Information Processing and Retrieval Programs." Senate Committee on Government Operations, 86th Congress, 2nd Session, Senate Doc. No. 113. Washington, D. C., U. S. Government Printing Office, 1960.

285. U. S. NAVY DEPARTIENT. "The Bureau of Ships Rapid Selector." Washington, D. C., Bureau of Ships, December 1959, 10 p.

286. U. S. MHVY DEPIRTMIENT. "Navy-Marine Corps Standard Subject Classification System." Washington, D. C., Ofice of the Secretary, October 30, 1959, 53 p., SECN/VINST P5210.11.

287. VAN DER WOLK, L. J. "Teletype et Microfiche Transparente dans les Relations entre Bibliothèques (Telex and Microcard Communication)," Bulletin des Bibliothèques de France, 4:11 (November 1959) 475-494. Abstracted in American Documentation, 12:1 (January 1961) 69. 
288. VERRY, H. R. "Rapid Selectors," O \& M Bulletin, London, 8:5 (October 1953) 43-46.

289. VESSEY, H. F. "Documentation Committee Report on Retrieval Systems." Farnborough (England), Royal Aircraft Establishment. Prepared for discussion at the Third Meeting of the Documentation Committee, Fourth General Assembly of the Advisory Group for Aeronautical Research and Development, The Hague, May 1954, 5 p.; AGARD/DOC/5.2. ASTIA doc. no. AD-68-199.

290. VICKERY, BRIAN C. "Some Comments on Mechanical Selection," American Documentation, 2:2 (April 1951) 102-107.

291. WALKER, FRED L., JR. "Blueprint for Knowledge," Scientific Monthly, 72:2 (February 1951) 90-101.

292. WARHEIT, I. A. "Machines and Systems for the Modern Library," Special Libraries, 48:8 (October 1957) 357-363.

293. WEBER, E. "A Suggestion for an Improved Data Banking System." Los Angeles, Calif., Benson-Lehner Corp., July 15, 1959, 29 p., Research Report 59-8, Contract Nonr-2666(00), Nr 048-135. ASTIA doc. no. AD-225 097 .

294. WESTERN RESERVE UNIVERSITY, Cleveland, Ohio. Contract AF 30(602)-1498, "Minicard Indexing Study."

PERRY, J., Report 1, March 1956, ASTIA doc. no. AD-91 781;

PERRY, J. and M. EGAN, Report 2, April 1956, ASTIA doc. no. AD-101 704;

PERRY, J., A. KENT, and M. EGAN, Report 3, May 1956, ASTIA doc. no. AD-96 889;

PERRY, J., Report 4, June 1956, ASTIA doc. no. AD-97 754.

295. "Westinghouse Automates Engineering Drawings," Managernent and Business Automation, 1:1 (January 1959) 19-25, 47-48.

296. WILD, JOHN J. "Predetermined Counters," Electronics, 20:3 (March 1947) 120-123.

297. WILLIAMS, T. J. and A. ROSE. "A Solution to the Problem of Storage and Availability of Chemical Literature," Journal of Chernical Education, 29:3 (March 1952) 146-147. 
298. WILSON, CARROLL L. Boston, Mass., Research Corporation, letter of April 3, 1940, to Dr. Vannevar Bush, Carnegie Institution of Washington, transmitting for comments the Howard-Wilson memorandum of April 1, 1940, Subject, Discussion of Possible Applications of the Rapid Selector. (Unpublished).

299. WILSON, LOREN R. "Integrated Card Handling and File Unit." Fort Wayne, Ind., Magnavox Corpany, May 31, 1960, 44 p., Report No. R332, Contract AF 30(635)12004; RADC TR 60-167. ASTIA doc. no. $A D-243065$.

300. WISE, CARL S. "Multiple Word Coding vs. Random Coding for the Rapid Selector," American Documentation, 3:4 (October 1952) 223-225.

301. WISE, CARL S. and JAMES W. PERRY. "Multiple Coding and the Rapid Selector," American Documentation, 1:2 (April 1950) 76-83.

302. WORSLEY, PETER K. "Data Retrieval With Especial Application to Use of Film Library Instantaneous Presentation (FLIP) in Literature Searching." Chapter in Modern Trends in Documentation, Proceedings of a Symposium held at the University of Southern California, April 1958, edited by Martha Boaz. New York, Pergamon Press, 1959, p. 70-73.

303. WORSLEY, PETER K., ROGER R. DITTMAN, EBERHARD WEBER, JUAN C. GRIMBERG, and PETER GARRETT. "A Study of the Fundamentals of Information Storage and Retrieval." Los Angeles, Calif., BensonLehner Corp., August. 20, 1959, 97 p., Final Report No. 417, Contract Nonr-2666(00). ASTIA doc. no. AD-229 709.

304. XEROX CORPORATION. "Final Engineering Report on the Development of a Minicard Viewer Processor MX-1993(XN-I)/G." Rochester, N. Y., June 1, 1961, 50 p., appendix. Submitted under Contract No. AF $30(602)-1523$ to Rome Air Development Center; RADC-TR-61-128. ASTIA doc. no. AD-260 073.

305. YALE UIIVERSITY. "Interim Report on the Development of the Rapid Selector." New Haven, Conn., May 1953, 53 p., Contract (RD)XG-796.

306. YALE UNIVERSITY. "The Nature and Use of the Yale Rapid Selector:" New Haven, Conn., October 1953, 13 p., Contract (RD)XG-796. 
307. YAIE UNIVERSITY. "Progress Report on the Yale Rapid Selector." New Haven, Conn., October 1953, 20 p.

308. YAIE UNIVERSITY. "Progress Report on the Yale Rapid Selector." New Haven, Conn., March 1954, 30 p.

309. YALE UNTVERSITY. "Report on the Demonstration of the Rapid Selector at Yale University." New Haven, Conn., May 18, 1955, $14 \mathrm{p}$.

310. YOUNG, GEORGE V., Inglewood, Calif., assignor of one-half to Peter J. Pohl, Manhattan Beach, Calif. "Video Image Frame Recording and Reproducing System," U. S. Pạtent 2,955,157; patented October 4, 1960; filed August 22, 1956. Class 178-6.6.

311. ZEISIG, H. C., JR. and P. T. MARTIN. "Commercially Available Punched-card Systems, Equipment and Supplies." Chapter 4 in Punched Cards, Their Applications to Science and Industry, edited by Casey and Perry. New York, Reinhold Publishing Corp., 1951. 
INDEX

abrasion: 38

abstracts: $3,10,16,17,22,26,41,46,66,83,85-87,94,105,107$, 109

access: 105

clues: 9

level: 8

(see also type of access)

accounting: 12, 15, 18

address: $4,8,10,34,37,87,94,95$

code-key: 106

file systems: 4

type selection and systems: 4, 15, 18, 26, 35-37, 40, 60, 78, 83,

(see also file)

$92,95-98,106,107,109$

adhesion: 108

adhesive-backed film: 93

aerial photographs: 3, 68, 109

Agriculture, Department of: 12, 21, 92

air-conditioning: 107

aircraft drawings: 92

Air Force, U.S.: 68

air traffic control: 34

alignment: 43

"American Documentation": 57

"American Journal of Photography": 12

ANFIS: $36,96,106$

Ampex Corporation: 34

AN/GSQ-II (v): (see Minicard)

ANV/GSQ-26: (see Minicard)

annotation: 47

(see also abstracts)

anticipator: 22

aperture

cards: $4,11,15,27,28,32,36,75,76,87,98,102,107-109$

card systerns: $36,90-95,98$

(see also magnetic aperture cards, microfiln aperture card systems)

Aperture Card System, Model D: 36, 98

applications: 2, 3, 6, 9, 25, 46, 103

archives: 11

Armed Services Technical Information Agency: 66

Mrrny Engineers, U.S.: 93 
aspect card: 83

ASTIA: (see Armed Services Technical Information Agency) atmosphere, controlled: 55, 57, 58

Automatic Catalog-Handbook: 47

Automatic Image Retrieval System: 35

description: 56, 57

(see also Appendix B)

Automatic Image Retriever: 97

automatic

processing

search

selection

(see information)

Automatic Unit Research Storage and Retrieval Device: 36 AutoTutor: 35,98

auxiliary equipment: 39,104

Avakian, Emil A.: 36

Avco Corporation: 36,96

banking: 12, 26

Battelle Memorial Institute: 113

Batten card: 83

Bell and Howell Company: 94

Bell Telephone Laboratories: 110

Benson-Lehner Corporation: 34, 35, 78, 98

Bibliofilm Service: 12

bibliographic identification: 5, 7, 8, 17, 40, 46, 86, 112 bibliographies: 6,16

binary representation: 25, 56, 57,61

Bishop, Charles: 104

blueprints: 3,92

bolometres: 12

books: 9, 11,26

Boolean manipulation: 32

Brewster, Sir David: 12

browsing: 55, 85, 101

Bryce, James W.: 12, 14, 15, 26

buffering: 59

Bureau of -...-: (see generic narne of Bureau)

Burkhard, C. A.: 28

Bush Comrnittee: 32,34

Bush Rapid Selector: (see Bush, Vannevar; Rapid Selector)

Bush, Vannevar: 15, 18, 19, 21, 27, 32, 35, 40, 57

business data: 12

Butterworth, Inc.: 30 
Cables and Insulating Materials Research Institute: 9

Callery Chemical Company: 109

cameras: (see microfilm)

canning: 28

capsule: 97

card

cutter: 52

punch: 47

reader: 52

stock: 37

thickness: 48

transport: 77,78

(see also type of card)

carrier cards: 16, 93

cassetts: 82,97

catalogues and cataloguing, 11, 101

categorization: $5,7,32,101$

cathode-ray tube: 96

Census, Bureau of: 40,48

Central Intelligence Agency: 23, 25, 93

Centre National de Recherche Scientifique de France: 63, 109

charts: $28,34,78,110$

(see also maps)

chemical literature: 6, 93, 109

Chicago, University of: 37

chip film and systems: 27, 34-37, 60-82, 94, 97, 103, 105, 111, 112

(see also Appendix B)

circuitry: 14, 43, 101

classification: $5,7,16,17,32,93$

(see also subject, specific classification scheme)

clay tablets: 9

climatology: 48

clutch control: 43

code: 34

field: 23

pattern: 19, 22, 23, 27

coded

file: 3

information: $8,10,18$

coding: 2, 4, 8, 10, 21-23, 30-32, 40, 41, 78, 93, 95, 99, 101, 107, 113

vocabulary: 85

Coile, Russell: 19

color film: 92

Commerce, Department of: 19, 21 


\section{communication and systems: 7, 102, 114}

(see also facsimile, remote, teletype, television, video) compact/compressed storage: 4, $11,32-34,95,102,103$ compartmentalization: 17, 101, 105 Computer Control Company, Inc.: 60 compressed: (see compact)

computer

application: 34, 48, 87, 95, 107 control: 97 installation: 68

content analysis: 8 continuous roll: (see roll) control film: 26 conventional

\section{approach: 7,33}

systems: 108

Coombs, John: 19, 22

coordinate indexing: 32,82

copy gate: 43,46

correspondence: 93

Cottrell, F. G.: 12

Council on Library Resources: 3, 96, 103, 113, 115

counting devices: 12

CRIS: $36,97,106$

cross-referencing: 16, 94, 105

cuneiforms: 9

cursors: 87

Czechoslovakia: 9

Dagron, Rene: II

damage: 107, 108

Dancer, John Benjamin: 11

DARE: 94, 95, 107

Data Bank: 35

description: 78-82

(see also "Is there a Doctor in the house?", Appendix B) data

display systems: 34

handling and processing: $5,6,18,31,32,77$

locating: 4

manipulation: 6

reduction: 4

retrieval: 5

storage: 9, 11, 103

(see also information, type of data) 
Davis, Helen M.: 17

Davis, Watson: 11, 12, 15-18, 32

decimal characters: 22

deep indexing: 95

Defense Logistics Service Center: 56

Department of -...-.: (see generic name of department)

descriptors: $32,46,66$

deterioration

general: 59

microbial: 37

(see also specific type of deterioriation involved)

developmental prospects: 102-115

devices: (see information)

Dewey Decimal Classification: 9, 16

diazo dye: 37

dictionary: 4

Diebold, Inc.: 56

digital data: 25, 66, 86, 87

(see also data)

direct access file: 96

directives: 49

directory: 4

dirt: 38,49

discrete

indexing: 19

unit records and systerns: $27,28,30,31,33,37,60-82,93$, $104+106$

dispatches: 11

display: 34

wheel: 78

document analysis: 2, 23

Documentary Storage and Retrieval System: 35

description: 90

(see also hppendix B)

documentation: 2, 6, 11, 15, 32, 113

Documentation of Molecular Spectroscopy System

description: 28,30

Documentation Institute of Science Service: (see Science Service)

document

frames: 19, 22, 23, 25, 34

identification: 5,19

recording: 9

retrieval devices: 3, 4, 15, 18, 25, 35-37, 106-108, 112

(see also image) 
documents and document collections: $3-6,8,18,19,23,26,30,63$, (see also information, libraries)

Draeger, Rupert H.: 12, 15, 17

drawings: $3,18,28,73,97,107,110$

(see also aircraft drawings.)

duplication: 11, 12, 18

(see also reproduction)

DuPont film

Chronar: 63

Mylar 4696: 76

dust: 38

EAM equipment: 76

Eastman Kodals Company: 18, 26, 27, 35, 56, 63, 68, 73, 90, 111

ECCETRON: 35

edge-notched cards: 28, 31, 78, 93, 108, 109

(see also E-Z Sort, Friden, McBee, punched cards)

Edual Laboratories: 10

Edual Laboratories for Philatelists: 10

electricity: (see electronics)

Electrofax printing: 59

(see also Radio Corporation of America)

electrographic pencil: $6 I$

electronics: 31-33, 49, 66, 107

(see also circuitry)

electrostatic printing: 90, 96

(see also Electrofax printing, xerography)

encoding: (see coding)

Encyclopaedia Brittanica: 12

engineering drawings: 28, 41, 46, 92, 102, 108, 110

(see also drawings)

Engineering Research Associates: 19, 21-23, 31

England: 11, 63

(see also Great Britain)

environnental conditions: 37, 38, 107

(see also atmosphere, controlled; deterioration)

equations: 18

equipment: $39,103-108$

characteristics: 2

development: 113

(see also type of equipment)

experience: (see system)

E-Z Sort cards: 92 
FACSI, Inc.

description of system: 31

facsimile

comrnunication: 40,102, 110, 112

reproduction: 2, 3, 33, 102

(see also communication, replica)

facts: 5

Fairchild Camera and Instrument Corporation: 36, 98 self-threading processor: 47

Federal Supply Code Classification: 92

feedback: 85

Ferranti Packard Electric Limited: 35, 98

fiber optics: 52

file

access points: 99

address: 34,106

arrangement: 4, 19, 33, 106, 107

expansion: 101

handling: 101

integrity: $3,47,105,110$

maintenance: $12,105,106$

manipulation: 10

merging: 93

organization: 73, 101, 102, 105, 113

rack: 80

reference approach: 4-7, 95

revision: 105, 106

segregation: 17

storage: 101

(see also document, information, item, type of file)

Filesearch: $35,98,101$

description: 49-55

(see also Appendix B)

filing-key code: 73

film: (see microfilm)

cards: $15,30,104$

cartridge: 97

Film Data Recording and Reading System: 34, 35, 98

film

life: $37,38,40$

matrix: 83

Film'N File, Inc.: (see Filmsort Company)

Filmorex system: 16, 27, 32, 35, 73, 106, 108, 109, 111

description: 63,66

(see also $\Lambda$ ppendix B) 
film printing machines: 14

Filmsort Company: 27, 28, 32

film transport: 17, 41

(see also high-speed transport)

filtering: 80,82

flashlamp: (see photoflash)

flexibility: 8

FLIP: 34, 35, 98

fluorescent recording: 37

flying-spot-scanner: 55, 59

FMA, Inc.: 35, 49

FOSDIC II: 35,108

description: 48,49

FOSDIC systems: 48

(see also Appendix B)

France: 11, 27, 48, 63, 66, 109

Friden

card: 52

card punch: 52

gating: 55

gelatin emulsions: 38

General Electric Company: 37

General Precision, Inc.: 36, 98

generic terms and search: 26, 107

geology and geological data: 30,61, 93

Germany: 12, 13, 30

Goldberg, Fmanuel: 12-15, 21, 26, 32

Goodale, Charles J.: 15, 26

graphic

data: 107

identity: 9

replica: 8, 18, 107, 110

representation: 3, 66, 101, 103, 112

graphs: 28

gray-scale: 46

Great Britain: 30

(see also England)

Green, John C.: 19, 21, 35

grid: 85

overlay: 86

Griffis Air Force Base: 63

half-tones: 46

handwritten records: 110 


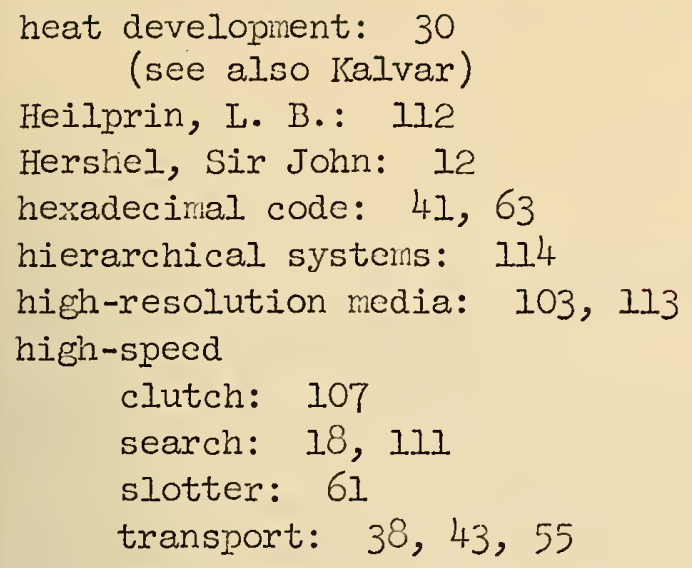


index

term cards: 83,86

terms: $5,40,60,85,87,109$

information

analysis: 7

automatic handling and mechanized devices: 2, 4, 6, 9, 11,12 , $14-19,22,27,32,34$, $38,66,68,97,101$,

classification: 7 $103,104,107,108,110$

(see also address, classification, code, data, document, file, item, mechanics, replica, retrieval, scientific information, search, selection, system, and various types of these and subheadings)

Information for Industry, Inc.: 36, 97

(see also Information Retrieval Corporation) information (cont.)

identification: $1,7,14$

organization: 7

processing: $3,5,9,27,101$

recording: 9,11

retrieval: $1,3-6,8-10,14,16,18,28,31,32,34,60,98,101$, $103-105,107,108,114$

(see also address, classification, code, data, document, file, item, mechanics, replica, retrieval, scientific information, search, selection, system, and various types of these and subheadings)

Information Retrieval Corporation: 36, 96

(see also Information for Industry, Inc.) information (cont.)

search: 1-3, 5, 7-9, 15, 16, 19, 22, 23, 31, 38, 40, 101, 102, 105,110

selection: $1-4,7-10,14,16,17,19,28,31,32,38,60,98$, $101-105,108,114$

storage: 1-3, 5-9, 14-17, 32, 37, 98, 106

(see also address, classification, code, data, document, file, item, mechanics, replica, retrieval, scientific information, search, selection, system, and various types of these and subheadings)

infrared spectral data: 28

input: (see generic type of input)

equipment: 39

insert cards: (see aperture cards)

Institute International de Documentation: 16 
integral indexing: 2, 8-11, 14, 15, 28, 30, 48, 61, 95, 99, 102, 109, 110

integrated search and selection: 112

intelligence data: 68

Intellofax system: 93

interfixing: 107

interlibrary loans: 102

(see also libraries)

International Business Machines Corporation: 35, 57, 86

International Conference on Scientific Information: 68

International Dewey Classification: (see Dewey Decimal Classification)

interrelationships: 14, 100

interrogator: 23,58

introduction: $1-8$

inviolate file: $1 \overline{1} 2$

"Is there a Doctor in the house?": 35

description: 78-82

(see also Data Bank, Appendix B)

Itek Laboratories: 114

item

characteristics: 100

classification: 99

identification: 7, 9, 99, 101

manipulation: 10

organization: 7

retrieval: 10, 14, 34

selection: $9,10,14,34$

storage: 99

(see also file, information)

James, Peter: 35, 57, 58

Kalfax/Kalvar: $30,47,87$

Kerr cell: 82

keyboard/keypunch input: 58, 59, 66, 73, 78, 97

keywords: 31, 95

Kodak: (see Eastman Kodak Company)

Kodamatic: 35,98

Kuipers, John V.: 68

labeling: 28

Langan, John: 27 


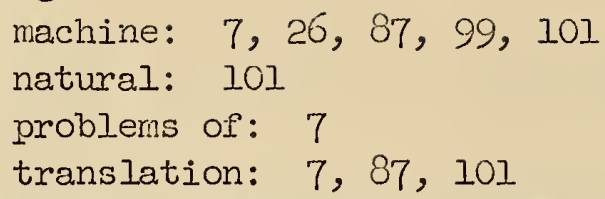

Large-Capacity Document Storage and Retrieval System: 35 description: 36,87 (see also Walnut, Appendix B)

large-scale systems: 114

libraries: 3, 7, 9, 11, 12, 15, 16, 18, 21, 47, 100, 102 (see also documents)

Library of Congress Classification: 9

light: 78

cells: 26

channel: 80

extinction: 22

raster: 56,60

scattering materials: 37

spectrum: 80

Lippman Emulsion EK 649 GH: 96

literature: (see information, scientific information, type of literature)

locator information: 4

Lodestar Reader: 35, 56, 98

logic

control: 40

manipulation: 100

London World's Fair: 12

long-range objectives: 2

look-up: $4,5,78$

Loquin, Marcel: 35,48

Loughren, Arthur V.: 27

Loughridge, Matthew H.: 25, 26

machine: (see information, man-machine communication, mechanics, and the rnachine's function by name)

Magnacard system: $76-78$

Magnaflux Corporation: 92

Magnascriber: 77,78

Magnavox Company: $34-36,76,97,98$

Magnavue system: 35,106

description: 76-78

(see also Appendix B) 
magnetic

aperture cards: 34,76

cards: 77

field: 82

recording and storage: 26, 34, 57, 58, 76, 77, 86, 87

tape: $32,37,48,49,57,59,104$

(see also video magnetic tape)

magneto-optical effect: 82

manipulation: (see information, mechanics)

man-machine communication: 2-8, 98, 99, 103

manual

filing: 66

retrieval: 93, 108

scanning: 4

search: 16

selection: 66

sorting: 66

maps: $3,34,46,73,78,107,110$

(see also charts)

masking: 19, 22, 48, 66

Massachusetts Institute of Technology: 18, 19, 21, 22, 27, 35, 98 Nast Development Company, Inc.: 35

master

file: 105, 110

film: $14,25,43,49$

index code: 10

matrix: (see gria)

McBee

cards: 10, 109

Keysort System: 28, 109

meaning: 7

mechanics, mechanization, and manipulation: 1-4, 6-12, 31, 49, 63,

(see also information)

95,109

media: 1, 8, 9, 14, 33

(see also high-resolution, storage)

WEDIA system: 36,97

liemex: 32

mernory plane: 96

Metricard: 36,98

microcard: 93, 102, 104

Microcite: 34, 35, 83, y9, 101, 105, 107

description: 83-86

(see also Appendix B)

microcopy: 97

microfiche: 4,104 
microfilm: 4, 10, 11, 15, 17-19, 22, 25, 27, 28, 31, 33, 34, 38, 43, $47,57,66,68,73,85,92,93,97,102-104,107,109,110$, 111

$16 \mathrm{~mm} .: 16,19,48,49,56,61,85,94,97,98,103$

$35 \mathrm{~mm} .: 17,19,22,40,41,46,49,52,55,61,87,96,97,103$

$70 \mathrm{~mm} .: \quad 60,103$

aperture card systems: 15, 37, 90, 92-95, 102, 109

cameras: $40,49,61,73$

cards: 33,63

processing: 113

viewers: 46, 66, 97, 104, 113

(see also aperture cards, film)

Microfilm Rapid Selector: (see Rapid Selector)

microform: 2, 4, 75, 104, 109, 110

microimage: 94,113

print: 90

(see also image)

Microimage Locator System: 36, 95, 106

microphotography and microfilming: 4, 11, 12, 15, 27, 92, 103

(see also photography)

micropositioning: 97

Micro-Research-Cards: $30,31,61$

Micro-Research System: 35, 108

description: $30,31,60-63$

(see also Appendix B)

microscope lens: 11

microstructure photographs: 3

Miehle Dexter Lawson Conpany: (see Filrusort Cormpany)

military specifications: 92

Minicard: 16, 27, 32, 35, 106, 108, 111

description of system: $68-76$

(see also Appendix B)

Minnesota líining and Manufacturing Company: 55, 60

(see also Filmsort Coimpany)

module: 87

moisture: 37,38

Mooers, Calvin: 32

Morse, R. S.: 26 
multiple

access: 110, 111

codes: 21

copies: 16, 105

entry file: 73,76

riling: 66

search: 55

search access: 94

search processing: 105

multiplexing: 102

Nyers, H. L.: 68

National Academy of Sciences: (see National Research Council)

National Bureau of Standards: 3, 22, 25, 28, 30, 34-36, 40, 48, 83, 95,106

National Cash Register Company: 18, 37

National Research Council: 28

National Science Foundation: 108, 109

National Teather Records Center: 48

Naval Photographic Interpretation Center, U.S.: 60

Navy, Department of: 19, 25, 40, 49, 112

Navy-Marine Corps Standard Subject Classification System Manual: 46, 52 needle-sorting and selection: $30,31,61,78,93,109$

needs: 3

neurology: 31

nonconventional

approach: 7

systems: $32,103,113$

nondestructive testing: 31

notes: 110

numerical data: 6,28

nunismatics: (see philately)

Office of Naval Research: 73

Orfice of Strategic Services: 27

Office of Technical Services: 19, 21

open-ended systems: 8, 113

optical recording: 57

optics: $31,49,82$

ordered files: 106

ortho A litho film: 108

Orthochromatic de Securite Microfile: 63

ortho contrast fi]m: 61

ONR: (see Office of Itaval Research)

oscillating hammer: 63 
oscillograph paper: 86

OSS: (see Office of Strategic Services)

OTS: (see Office of Technical Services)

output: (see generic type of output)

packaging: 28

packing density: 38, 41

paleontology: 30

paper stock: 38

parallel search: 59,61, 66, 75, 78, 101, 105

(see also multiplexing)

Paris, Siege of: 11

parity check: 52, 59, 77

Patent Office, British: 10

Patent Office, U.S.: 25, 30, 32

patents: $9,10,14$

peek-a-boo

card punch: 85,86

cards: $83,85,86$

systerns: 83

performance specilications: 2

permuted title index: 7

Petroleurn Research Corporation: 30, 35, 61, 108, 110

petroleum resources: 30

philately: 10

photocells: 12-14, 16, 19, 22, 23, 26, 43, 47, 66

(see also scanning)

photochromic recorảing: 37

Photo Devices, Ine.: 35,60

Model 1088B Canera: 60

photoelectric techniques: 17

(see also photocells)

photoflash: 22

photographic card: 61

Photographic Digital Reader-Recorder: 27

"Photographic News": 12

photographic output: 112

photographs: 10, 13, 19, 26, 26, 73, 90, 107

(see also aerial photographs, inages, microstructure photographs, pictoria] data)

photography: 8, 11, 12, 14, 16, 17, 22, 23, 26, 27, 31, 32, 37

(see also microphotography)

Photo-magnetic Systern: 35

description: 57, 58

(see also Appendix B)

photoprinting: 25 
pictorial data and infornation: 3, 18

(see also photograpins)

pigeon, homing: 11

plugboard: 49

pneunatic system: 77

pocket card: 9

polarized light: 82

Polaroid Land film: 86

police departments: 28

pover supply: 22

predictive search: 40

prematurity: 2

primary index terms: 85

Print-a-Pix: 96

printing, contact: 30

processing: (see information)

protection of media: 37, 104

prototype: $47,86,97,107$

publications: 15

punched cards: $10-12,16,17,23,27,28,30,32,33,41,43,48,57$, $60,61,66,83,92-95,106-108,111$

(see also edge-notched cards)

Punched Cards, Their Application to Science and Industry: 90

punched hole code: 94

punched paper tape: 12, 73, 95, 97

punch equipment: 23

Rabinow, Jacob: 22

Radio Corporation of America: 34, 35, 58

Ramo-Wooldridge: (see Thompson Ramo Wooldridge, Inc.)

random

access: $86,105,106$

location: 105

randomly organized files: 105, 106

Rapid Access Look-up System and projector: 35, 98

rapid direct access: 105, 106

Rapid Random Access Projector: 35

Rapid Selector: 15, 18, 19, 21-23, 25, 26, 32, 35, 57, 98, 107, 108, 111,112

description: $40-48$

(see also Appendix B)

RCA: (see Radio Corporation or Arerica)

Reader-Finder: 35,98

reading devices: 38

Recall Film Index System: 32

description: 30 
recognition match: 19

Recordak Corporation: 35, 56, 98

MRD2a Camera: 56

recording

format: 23

indicia: 4

records: 11, 12, 14, 18, 26, 99, 110

remote communication: 110

replica

copies: 3,8, 9, 14, 15, 22, 32, 34, 46, 95, 107, 110, 115

copy retrieval devices: 18

recording: 19

retrieval: 31, 32

(see also graphic)

reports: 5,46

reproduction: 9

(see also duplication)

research and development: 2

Research Corporation: 18

Research Information Center: 3

resolution: 110

retrieval

hooks: 5,7

speed: 102

systems: $8,10,12,15$

(see also information, type of retrieval)

retrospective search: 18, 32

RICASIP: (see Research Information Center)

Richardson Static Card Reader: 60

Rocky Mountains: 30, 31

Rocky Mountains Geology Library: 61

rogues' galleries: 28

role indicators: 46,107

roll film and systems: 34, 35, 37, 40-60, 98, 103, 104, 112 (see also Appendix B)

Rome Air Development Center: 63, 66, 68, 76, 111

Rose, A.: 93

Rotkin, Israel: 22

Royal McBee: (see McBee)

Ruggles, Melville J.: 47

Ruggles, Richard: 25

Russian language: 100

sales: 26

Samain, Jacques: 27, 63 
sampling: 8

scanning and scamning grid: 14, 27, 66

(see also flying-spot-scanner, grid)

screening: 100

"Science": 15

Science Service: 15, 17, 19

scientific information: $1,2,6,11,12,15,18,19,32,34$

(see also information)

scratching: 38

scroll film: 33, 97

search

complexity: 114

easel: 86

entry record: 100

equipment: 39

integrity: 105

key: 66

plate: 13,14

prescription: 5, 9, 22, 37, 43, 59, 99-102, 105, 106

prescription approach: $4-7$

procedure: 22,100

products: 102

request: 5 ,

requirements: 41

scheduling: 101

selectivity: 102

speed: 102

strategy: 101, 102

terms: 4

time: 23

type selection and systems: 4, 6-8, 15, 23, 26, 35-37, 40-90, 105, 106,108

(see also file, information, type of search)

searching systerns: 4

SECNAVINST P5210.11: (see Navy-Marine Corps Standard Subject

Classification System Manual)

security file: 105

(see also master file)

Seide11, Atherton: 12, 15, 27 
selection

code: 19, 22, 23, 26, 38, 43, 80, 106, 107, 113

code capacity: 113

code pattern: 82

code recording: 113

code storage: 113

criteria: 5, 7-9, 14, 16, 19, 22, 23, 26, 30, 99-102, 106, 107

gate: 46

logic: $102,107,114$

systems: $8,12,14,15,18,31,99,110,115$

(see also code, information, type of selection)

selenium cells: 12

semi-automatic selection: 61

Senate Committee on Government Operations: 6

sequential filing: 108

serial

access: 106

arrangement: 106

search: $43,49,58,59,66,75,98,104,105$

Seventy Millimeter Selector: 35

description: 60

(see also Appendix B)

Shannon, Claude: 19

Shaw, Ralph R.: 2l, 35

sheet or strip film and systems: 4, 35-37, 82-90, 103, 105

(see also Appendix B)

shipboard: 92

Ships, Bureau of: 25, 35, 40, 46, 49, 108, 111,112

silver-halide: 37

simultaneous

access: 3

search: 112

selection: 112

slides: (see photographs, photography)

Slosson, Edwin E.: 12, 15

slotted card: 61

slotting: 73

Social security cards: 26

"Society for Nondestructive Testing, Journal": 31, 93

sorting: 5, 12, 93

box: 61

mechanism: 16

special-purpose systems: 3

specific terms and search: 26, 107

spectrogram: 30

splicing: 105, 106 
Staats, Henry iv.: 35, 90

stability of media: 37,95

stamps: (see philately)

state-of-the-art: 2, 34, 100, 102-115

statistical

data: 12, 15, 18, 25, 26

machines: 12

Steinhardt, Lawrence: 19

step and repeat camera: 61,96

storage

conditions: 37

devices: 95

economy: 102

levels: 85

life: 37

materials: 7, 9, 27, 37-39, 41, 99, 103-108, 113

space: 77

(see also information, media, type of storage)

strip or sheet film and systems: 4, 35-37, 82-90, 103, 105

(see also Appendix B)

strobotron: 19

Stuart, R.: 25, 26

subject

analysis: 8 , i13

classification: 16,17

content: $3,23,34,105$

identification: 6

submarines: 102

Sumaria: 9

superimposed

image: 78

random coding: $82,99,106$

system

advantages: 104, 112

analysis: $2,110,114$

applications: 2

availability: 103

benerits: 110

capacity: 102

characteristics: 37-98, 102

cormarisons: 110

compatibility: 102, 112

costs: 8, 107, 110

design considerations: $23,98-103,112-114$

(more) 
system (contd.)

development: 40

difficulties: 106

disadvantages: 103

efficiency:

evaluation criteria: $7,39,110,111$

experience: 108-112

flexibility: 40

index: 8

objectives: 103

operating conditions: 110

operations: 113

planning: 2

requirements: 2, 5, 38, 47, 112, 113

selectivity: 106

utilization: 12, 18, 23, 32, 100, 102, 103, 112, 114

(see also information, type of system, subheads and individual entries, Appendix B)

tabulating

card: 27

equipment: 10

Target Map Coordinate Locator: 36,98

teaching machines: 98

technical literature: 1, 5

(see also scientific information)

technological application development: 2

Telecard system: 36,98

teletype communication: 102

television

recording: 59

techniques: 98

(see also video)

term entry card: 85

terminology: 100

textual information: $3,18,22,40,46,101,110$

thermal stability: 37

thermoelectric pile: 12

thermoplastic recording: 37

Thormpson Ramo Wooldridge, Inc.: 36, 98

title records: 28

Townsend, Leonard G.: 15, 26

TPR: (see thermoplastic recording)

traditional approach: 7

translation: (see language) 
tub file: 56

Tyler, A. W.: 68

typewriter: 16,48

UDC: (see Universal Decimal Classification)

ultraviolet exposure: 30

(see also Kalvar)

Union of Soviet Socialist Republics: 47,63

United States -..--: (see generic name of organization)

United States Industries, Inc.: 35

uniterms: 32

Universal Decimal Classification: 9

University of -...-. (see proper name)

up-dating: 8, 101, 113

usage and user requirements: 2, 8

USI: (see United States Industries, Inc.)

vacuum drums: 77

Verac 903: 36, 96, 105, 106

Video File System: 35

description: $58-60$

(see also Appendix B)

video

communication systems: 96

magnetic tape: 34,59

techniques: 58,96

(see also television)

Viever Reproducer, Models $\mathrm{C}$ and $\mathrm{E}: 35,98$

vievers: (see microfiln)

visual image display device: 76

vocabulary code cards: 63

Talnut: 35,105

description: 86,87

(see also Appendix B)

Waterman, Alan T.: 6

wear: 38, 107, 108

Weather Bureau, U.S.: 35, 48

Western Design \& Electronics: 35

Whirlirind computer: 27

Villiams, T. J.: 93

word card: 16

World War II: 19

Wright-Patterson $\Lambda$ ir Force Base: 92 
xerography: 94, 97

(see also electrostatic printing)

Yale University: $23,25,35,40,57$ 


\begin{tabular}{|c|c|c|c|c|c|}
\hline & & TOTAL SYST & & & \\
\hline & Cost & $\begin{array}{l}\text { Approximate } \\
\text { Volume }\end{array}$ & $\begin{array}{l}\text { Approximate } \\
\text { Cost }\end{array}$ & Name & Type \\
\hline & $\$ 8,000-$ & $350 \mathrm{ft} .3$ & $\$ 80,000-$ & $\begin{array}{l}\text { Rapid Selector } \\
\text { p. } 40-48\end{array}$ & continuous roll \\
\hline & --- & $150 \mathrm{ft}$. & $\$ 112,000-$ & $\begin{array}{l}\text { FOSDIC II } \\
\text { p. } 48-49\end{array}$ & continuous roll \\
\hline & $\$ 4,000-$ & $150 \mathrm{ft}$. & $\$ 115,000-$ & $\begin{array}{l}\text { FileSearch } \\
\text { p. } 49-55\end{array}$ & continuous roll \\
\hline & $\$ 3,400-$ & INA & $\$ 45,400-$ & $\begin{array}{l}\text { Automatic Image Retrieval } \\
\text { System p. } 56-57\end{array}$ & continuous roll \\
\hline & $\$ 3,500-$ & $320 \mathrm{ft}$. & $\$ 80,000-$ & $\begin{array}{l}70 \mathrm{~mm} \text {. Selector } \\
\text { p. } 60\end{array}$ & continuous roll \\
\hline & INA & INA & INA & $\begin{array}{l}\text { Video File System } \\
\text { p. } 58-60\end{array}$ & continuous roll \\
\hline & -- & INA & INA & $\begin{array}{l}\text { Photo-magnetic System } \\
\text { p. } 57-58\end{array}$ & continuous roll \\
\hline & --- & INA & INA & $\begin{array}{l}\text { Micro Research System } \\
\text { p. } 60-63\end{array}$ & discrete chip \\
\hline & $\begin{array}{r}\$ 800-(3 \mathrm{M} \\
\text { viewer- } \\
\text { printer }) \\
\end{array}$ & $75 \mathrm{ft}$. & $\begin{array}{c}\$ 8,300- \\
\text { (FOB-Paris) }\end{array}$ & $\begin{array}{l}\text { Filmorex } \\
\text { p. } 63-66\end{array}$ & discrete chip \\
\hline $\begin{array}{l}\text { dupli- } \\
\text { iewer; }\end{array}$ & $\begin{array}{l}\text { included in } \\
\text { total cost }\end{array}$ & $1200 \mathrm{ft}^{3}$ & $\$ 2,000,000-$ & $\begin{array}{l}\text { Minicard } \\
\text { p. } 68-76\end{array}$ & discrete chip \\
\hline & $\begin{array}{l}\text { included in } \\
\text { total cost }\end{array}$ & $2000 \mathrm{ft}^{3}$ & $\$ 400,000-$ & $\begin{array}{l}\text { Magnavue } \\
\text { p. } 76-78\end{array}$ & discrete chip \\
\hline & INA & INA & INA & $\begin{array}{l}\text { Data Bank ("Is there a } \\
\text { doctor in the house?") } \\
\text { p. } 78-82\end{array}$ & discrete chip \\
\hline & INA & $250 \mathrm{ft}$. & INA & $\begin{array}{l}\text { Microcite } \\
\text { p. } 83-86\end{array}$ & sheet or strip \\
\hline & INA & INA & INA & $\begin{array}{l}\text { Walnut } \\
\text { p. } 86-87\end{array}$ & sheet or strip \\
\hline & INA & INA & $\$ 60,000-$ & $\begin{array}{l}\text { Documentary Storage and } \\
\text { Retrieval System } \\
\text { p. } 90 \\
\end{array}$ & sheet or strip \\
\hline & Cost & $\begin{array}{c}\text { Volume } \\
\text { Approximate }\end{array}$ & $\begin{array}{c}\text { Cost } \\
\text { Approximate }\end{array}$ & Name & Type \\
\hline
\end{tabular}






\section{U.S. DEPARTMENT OF COMMERCE \\ Luther H. Hodges, Secreiary \\ NATIONAL BUREAU OF STANDARDS \\ A. V. Astin, Director}

\section{THE NATIONAL BUREAU OF STANDARDS}

The scope of activities of the National Bureau of Standards at its major laboratories in Washington, D.C., and Boulder, Colorado, is suggested in the following listing of the divisions and sections engaged in technicai work. In general, each section carries out specialized research, development, and engineering in the field indicated by its title. A brief description of the activities, and of the resultant publications, appears on the inside of the front cover.

\section{WASHINGTON, D.C.}

Eiectricity. Resistance and Reactance. Electrochemistry. Electrical Instruments. Magnetic Measurements. Dielectrics. High Voltage.

Metroiogy. Photometry and Colorimetry. Refractometry. Photographic Research. Length. Engineering Metrology. Mass and Scale. Volumetry and Densimetry.

Heat. Temperature Physics. Heat Measurements. Cryogenic Physics. Equation of State. Statistical Physics.

Radiation Physics. X-ray. Radioactivity. Radiation Theory. High Energy Radiation. Radiological Equipment. Nucleonic Instrumentation. Neutron Physics.

Analytical and Inorganic Chemistry. Pure Substances. Spectrochemistry. Solution Chemistry. Standard Reference Materials. Applied Analytical Research. Crystal Chemistry.

Mechanics. Sound. Pressure and Vacuum. Fluid Mechanics. Engineering Mechanics. Rheology. Combustion Controls.

Poiymers. Macromolecules: Synthesis and Structure. Polymer Chemistry. Polymer Physics. Polymer Characterization. Polymer Evaluation and Testing. Applied Polymer Standards and Research. Dental Research.

Metallurgy. Engineering Metallurgy. Microscopy and Diffraction. Metal Reactions. Metal Physics. Electrolysis and Metal Deposition.

Irorganic Solids. Engineering Ceramics. Glass. Solid State Chemistry. Crystal Growth. Physical Properties. Crystallography.

Building Research. Structural Engineering. Fire Research. Mechanical Systems. Organic Building Materials. Codes and Safety Standards. Heat Transfer. Inorganic Building Materials. Metallic Building Materials.

Applied Mathematics. Numerical Analysis. Computation. Statistical Engineering. Mathematical Physics. Operations Research.

Data Processing Systems. Components and Techniques. Computer Technology. Measurements Automation. Engineering Applications. Systems Analysis.

Atomic Physics. Spectroscopy. Infrared Spectroscopy. Solid State Physics. Electron Physics. Atomic Physics.

Instrumentation. Engineering Electronics. Electron Devices. Electronic Instrumentation. Mechanical Instruments.

Basic Instrumentation.

Physical Chemistry. Thermochemistry. Surface Chemistry. Organic Chemistry. Molecular Spectroscopy. Molecular Kinetics. Mass Spectrometry.

Office of Weights and Measures.

\section{BOULDER, COLO.}

Cryogenic Engineering. Cryogenic Equipment. Cryogenic Processes. Properties of Materials. Cryogenic Technicul Services.

\section{CENTRAL RADIO PROPAGATION LABORATORY}

lonosphere Research and Propagation. Low Frequency and Very Low Frequency Research. Ionosphere Research. Prediction Services. Sun-Earth Relationships. Field Engineering. Radio Warning Services. Vertical Soundings Research.

Radio Propagation Engineering. Data Reduction Instrumentation. Radio Noise. Tropospheric Measurements Tropospheric Analysis. Propagation-Terrain Effects. Radio-Meteorology. Lower Atmosphere Physics.

Radio Standards. High Frequency Electrical Standards. Radio Broadcast Service. Radio and Microwave Materials. Atomic Frequency and Time Interval Standards. Electronic Calibration Center. Millimeter-Wave Research. Microwave Circuit Standards.

Badlo Systems. Applied Electromagnetic Theory. High Frequency and Very High Frequency Research. Modulation Research. Antenna Research. Navigation Systems.

Upper Atmosphere and Space Physics. Upper Atmosphere and Plasma Physics. Ionosphere and Exosphere Scatter. Airglow and Aurora. Ionospheric Radio Astronomy.

\section{RADIO STANDARDS LABORATORY}

Radio Physics. Radio Broadcast Service. Radio and Microwave Materials. Atomic Frequency and Time-Interval Standards. Millimeter-Wave Research.

Circult Standards. High Frequency Electrical Standards. Microwave Circuit Standards. Electronic Calibration Center. 


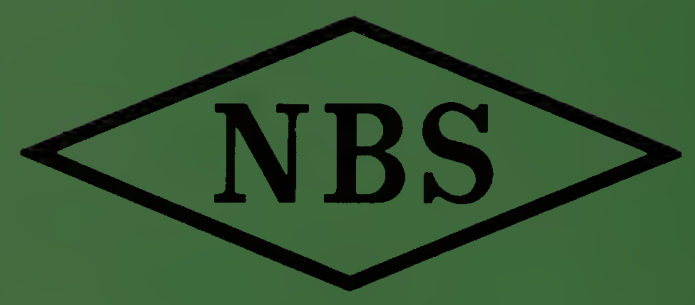

